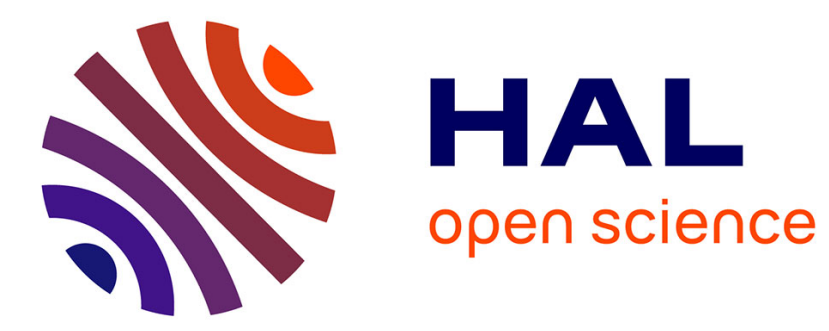

\title{
Pompage optique doublement sélectif en vitesses
}

M. Pinard, L. Julien, F. Laloë

\section{To cite this version:}

M. Pinard, L. Julien, F. Laloë. Pompage optique doublement sélectif en vitesses. Journal de Physique, 1982, 43 (4), pp.601-629. 10.1051/jphys:01982004304060100 . jpa-00209431

\section{HAL Id: jpa-00209431 https://hal.science/jpa-00209431}

Submitted on 1 Jan 1982

HAL is a multi-disciplinary open access archive for the deposit and dissemination of scientific research documents, whether they are published or not. The documents may come from teaching and research institutions in France or abroad, or from public or private research centers.
L'archive ouverte pluridisciplinaire HAL, est destinée au dépôt et à la diffusion de documents scientifiques de niveau recherche, publiés ou non, émanant des établissements d'enseignement et de recherche français ou étrangers, des laboratoires publics ou privés. 
Classification

Physics Abstracts

32.80B -42.65

\title{
Pompage optique doublement sélectif en vitesses
}

\author{
M. Pinard, L. Julien et F. Laloë \\ Laboratoire de Spectroscopie Hertzienne de l'E.N.S., 24, rue Lhomond, 75231 Paris Cedex 05, France
}

(Reçu le 6 novembre 1981, accepté le 21 décembre 1981)

\begin{abstract}
Résumé. - La méthode du pompage optique sélectif en vitesses est étendue à la sélection de deux composantes de la vitesse des atomes, grâce à l'utilisation de deux faisceaux de pompage de directions différentes, et à l'observation d'effets croisés non linéaires, proportionnels à l'intensité de chacun des faisceaux. Un troisième faisceau, coplanaire avec les deux précédents, sert à la détection, les trois faisceaux étant issus du même laser. On obtient par cette méthode toute une série de signaux correspondant aux différentes observables atomiques (population, orientation, alignement) que l'on peut créer ou détecter, suivant le choix de la polarisation de chacun des faisceaux. On discute dans cet article la nature et la forme de ces signaux, ainsi que les conditions géométriques dans lesquelles ils ne sont pas élargis par l'effet Doppler. On décrit enfin une expérience réalisée avec des atomes de néon métastables où un certain nombre de ces signaux ont été observés.
\end{abstract}

\begin{abstract}
The velocity selective optical pumping(V.S.O.P.) method is generalized to the selection of two components of the atomic velocities. Two pump beams with different directions are used and the signal obtained is due to crossed non-linear effects, proportional to both beam intensities. A third coplanar beam is used for detection, all three beams originating from the same laser. Theory predicts that a series of different signals can be obtained by this method, corresponding to various atomic observables (population, orientation, alignment) which are pumped or detected, depending on the beam polarizations. We discuss the shape of the various signals as well as the geometrical conditions leading to Doppler-free curves. Finally experiments with metastable neon atoms are described; their results agree with the theory.
\end{abstract}

Introduction. - La mise au point de lasers monomodes a ouvert la voie à de nouvelles méthodes spectroscopiques utilisant la sélectivité en vitesse de l'excitation produite par de telles sources. La plus connue de ces méthodes est celle de l'absorption saturée, qui fait intervenir des effets de saturation d'une transition optique, et qui a donné lieu à de nombreuses applications [1]. Dans le cas où les deux niveaux de la transition optique étudiée ont des durées de vie très différentes et possèdent une structure (Zeeman par exemple), on peut utiliser la méthode du pompage optique sélectif en vitesses [2] dans laquelle les effets de saturation optique sont négligeables.

Rappelons le principe et les caractéristiques de cette dernière méthode, qui permet de créer, dans un niveau fondamental ou métastable donné, une observable déterminée, corrélée aux variables externes des atomes. On considère un système atomique à deux niveaux e et $\mathrm{g}$ de durées de vie très différentes : le niveau $g$ est fondamental ou métastable, tandis que le niveau e a une durée de vie d'origine radiative beaucoup plus courte $\tau_{\mathrm{e}}=\Gamma^{-1}$. Le niveau g, comme éventuellement le niveau e, comprend plusieurs sousniveaux Zeeman. Les atomes, en phase gazeuse, sont pompés optiquement par un faisceau lumineux issu d'un laser monomode, dont la fréquence $\Omega_{\mathrm{L}} / 2 \pi$ est voisine de la fréquence $\Omega_{0} / 2 \pi$ de la transition atomique $\mathrm{e} \leftrightarrow \mathrm{g}$. Le faisceau de pompage est étendu de façon à ce que, sous son effet, le système atomique évolue en « régime de pompage » [3], [4], [5]. Il faut pour cela que les cohérences optiques créées par l'excitation entre les niveaux e et $\mathrm{g}$ restent à tout instant négligeables. Le signal obtenu dans ces conditions n'est alors dû qu'aux observables créées par pompage optique dans le seul niveau g.

Plaçons-nous par exemple dans le cas où le faisceau de pompage, polarisé circulairement, crée dans le niveau g une orientation $\left\langle J_{z}\right\rangle$ (différence de populations entre sous-niveaux Zeeman); celle-ci est dirigée le long de la direction $\mathrm{O} z$ de propagation du faisceau. Si la largeur en fréquence de l'excitation est beaucoup plus petite que la largeur du profil Doppler de la 
transition atomique utilisée, seuls sont orientés par le pompage les atomes ayant sur l'axe $\mathrm{O} z$ une composante de vitesse qui vérifie, à $\Gamma$ près, la condition :

$$
\Omega_{\mathrm{L}}-\Omega_{0}=k v_{z}
$$

où $k$ est le module du vecteur d'onde de la lumière incidente. Le pompage introduit donc dans le niveau $\mathrm{g}$ une forte corrélation entre les variables internes atomiques (ici $\left\langle J_{z}\right\rangle$ ) et les variables externes $\left(v_{z}\right)$. La mesure du dichroïsme circulaire de la vapeur permet d'obtenir un signal proportionnel à l'orientation des atomes [6]. Suivant le schéma bien connu en absorption saturée, on peut utiliser pour la détection un faisceau sonde polarisé circulairement, issu du même laser monomode, et se propageant dans la direction opposée à celle du faisceau de pompage. Il détecte l'orientation des atomes de vitesse longitudinale $-\left(\Omega_{\mathrm{L}}-\Omega_{0}\right) / k$.

A résonance, le faisceau de pompage et le faisceau sonde interagissent avec les mêmes atomes de vitesse longitudinale $v_{z} \simeq 0$, et l'on obtient un signal d'orientation. Hors résonance par contre $\left(\left|\Omega_{\mathrm{L}}-\Omega_{0}\right|>\Gamma\right)$, les deux faisceaux interagissent avec des atomes de vitesses longitudinales différentes, et le signal d'orientation est nul. Quand on balaie la fréquence du laser, le signal d'orientation est donc une raie étroite centrée sur la fréquence atomique, et correspondant aux atomes de vitesse longitudinale quasi nulle.

Dans le raisonnement précédent, nous avons implicitement négligé l'influence des collisions, ou supposé qu'elles détruisent totalement $\left\langle J_{z}\right\rangle$. En présence de collisions non totalement dépolarisantes pour le niveau g, le signal d'orientation comporte également une composante large (sa largeur peut être comparable à la largeur Doppler) liée aux atomes qui ont été pompés alors qu'ils avaient la vitesse longitudinale $v_{z}$ mais qui, après collision, sont détectés alors qu'ils ont la vitesse longitudinale $-v_{z}$. L'étude du profil du signal obtenu, lorsqu'on balaie la fréquence du laser, permet d'obtenir des renseignements sur les collisions subies par les atomes dans la cellule. On peut ainsi remonter à différents paramètres caractéristiques de ces collisions : sections efficaces de collision [7], paramètres de conservation de l'observable étudiée lors de la collision, noyau intégral de collision $[8,9,10,11,12]$, etc...

Cependant, les expériences décrites ci-dessus ne permettent de sélectionner que l'une des composantes de la vitesse des atomes, celle qui est portée par l'axe $\mathrm{O} z$ de propagation commun aux deux faisceaux. Tous les effets que l'on peut étudier par cette méthode, en particulier ceux liés aux collisions, sont moyennés sur les deux autres composantes $v_{x}$ et $v_{y}$.

Le but de cet article est de montrer qu'il est possible de généraliser la méthode du pompage optique sélectif en vitesses de façon à sélectionner davantage de composantes de la vitesse des atomes. En particulier, l'utilisation d'un effet de pompage croisé entre deux faisceaux de pompage non colinéaires permet de sélectionner deux composantes de cette vitesse. Nous avons mis en évidence expérimentalement un tel effet, et calculé les signaux qu'on peut attendre dans une telle expérience.

1. Principe de la méthode. - 1.1 Double SÉLECTION EN VITESSES. - Considérons une expérience où l'on utilise deux faisceaux de pompage $P_{1}$ et $P_{2}$ et un faisceau de détection $\mathrm{D}$, tous trois issus du même
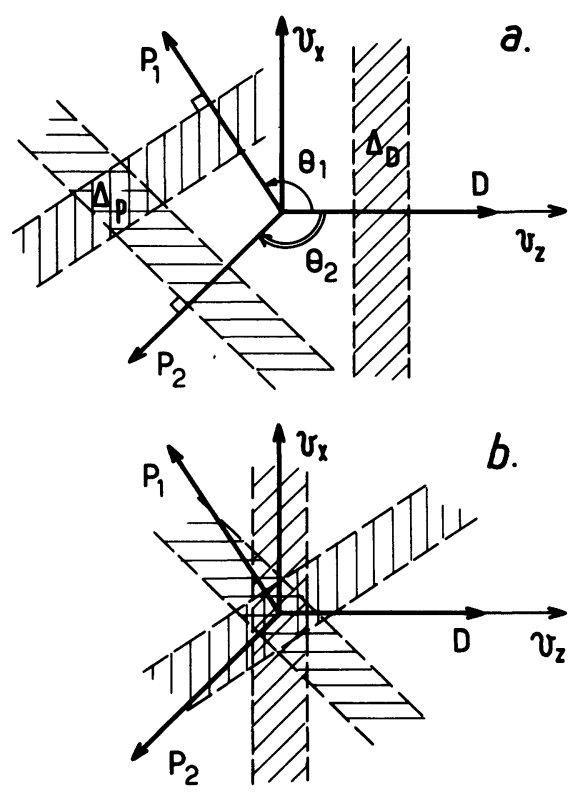

Fig. 1. - Schéma dans l'espace des vitesses associé aux atomes; pour un écart à résonance donné, à chacun des faisceaux de pompage $P_{1}$ et $P_{2}$ est associée une bande hachurée (perpendiculaire à ce faisceau) déterminant les vitesses des atomes qui sont en interaction résonnante avec lui; $\Delta_{\mathrm{p}}$, intersection de ces deux bandes, est le domaine correspondant aux atomes simultanément en interaction avec les deux faisceaux de pompage. De même, le faisceau de détection $D$, coplanaire avec $P_{1}$ et $P_{2}$, interagit de façon résonnante avec les atomes dont la vitesse tombe dans la bande hachurée $\Delta_{\mathrm{D}}$. La figure $1 a$ représente le cas où la fréquence du laser n'est pas exactement centrée sur la résonance atomique : les domaines $\Delta_{\mathrm{P}}$ et $\Delta_{\mathrm{D}}$ ne se recouvrent donc pas. La figure $1 b$ représente le cas où la fréquence du laser est parfaitement centrée sur celle-ci : un recouvrement important des domaines intervient.

[Figure 1 shows, in velocity space, the regions corresponding to atoms interacting with pumping beams $P_{1}$ and $P_{2}$ (hatched bands perpendicular to $P_{1}$ and $P_{2}$ ), or with both beams (doubly hatched area $\Delta_{\mathrm{P}}$ ). Similarly, the detection beam $\mathrm{D}$ selects atoms with velocity falling inside a band $\Delta_{\mathrm{D}}$ perpendicular to $\mathrm{D}$. In figure $1 a$, it is assumed that the laser is off-resonance, so that $\Delta_{\mathrm{P}}$ and $\Delta_{\mathrm{D}}$ do not overlap. In figure $1 b$, it is assumed that the laser frequency is centred exactly at the atomic resonance frequency, so that all three bands intersect around the origin and a Doppler-free signal is obtained.] 
laser monomode balayable. Dans la cellule contenant les atomes, les trois faisceaux sont coplanaires mais non colinéaires. La sélection en vitesses qui résulte de cette géométrie est schématisée sur la figure 1, qui représente l'espace des vitesses associé aux atomes. Les atomes en résonance avec le faisceau $\mathrm{P}_{1}\left(\right.$ resp. $\left.\mathrm{P}_{2}\right)$ ont la projection $v_{1}$ (resp. $v_{2}$ ) de leur vitesse le long de $P_{1}$ (resp. $P_{2}$ ) qui vérifie la relation :

$$
\left|\Omega_{\mathrm{L}}\left(v_{1} / c\right)-\left(\Omega_{\mathrm{L}}-\Omega_{0}\right)\right| \lesssim \Gamma .
$$

L'extrémité du vecteur vitesse de ces atomes est donc située dans une bande, hachurée sur la figure 1, perpendiculaire à $\mathrm{P}_{1}\left(\right.$ resp. $\left.\mathrm{P}_{2}\right)$. Les atomes en résonance à la fois avec les deux faisceaux de pompage ont l'extrémité de leur vecteur vitesse située dans un petit domaine $\Delta_{\mathrm{P}}$ doublement hachuré, intersection des deux bandes associées à $P_{1}$ et $P_{2}$. De la même façon, les atomes en résonance avec le faisceau de détection $\mathrm{D}$ ont la projection de leur vitesse sur $\mathrm{O} z$ qui vérifie une condition analogue à (1). L'extrémité de leur vecteur vitesse se trouve donc située dans la bande hachurée $\Delta_{\mathrm{D}}$ perpendiculaire à $\mathrm{D}$ représentée sur la figure 1 .

Hors de la résonance exacte, c'est-à-dire lorsque $\left|\Omega_{\mathrm{L}}-\Omega_{0}\right| \gg \Gamma$ et dans la géométrie considérée sur la figure, les zones $\Delta_{\mathrm{P}}$ et $\Delta_{\mathrm{D}}$ correspondant aux atomes soit " doublement pompés " soit détectés n'ont pas de recouvrement, comme représenté sur la figure $1 a$, et aucun atome n'est en interaction résonnante avec tous les faisceaux. A résonance cependant, et comme représenté sur la figure $1 b$, un tel recouvrement se produit et le petit domaine triplement hachuré situé au centre de la figure définit un ensemble d'atomes interagissant simultanément avec les trois faisceaux. On sélectionne ainsi les atomes de vitesse quasi nulle dans le plan des faisceaux (la composante $v_{y}$ de la vitesse, perpendiculaire à ce plan, ne joue aucun rôle).

1.2 Pompage optique À DeuX faisceauX. - Pour obtenir cette double sélection sur la vitesse des atomes, il est nécessaire d'observer un " effet de pompage croisé » entre les deux faisceaux $P_{1}$ et $P_{2}$, c'est-à-dire par exemple un effet en $I_{1} \times I_{2}$ où $I_{1}$ et $I_{2}$ sont leurs intensités respectives (effet de "saturation croisée "). Différents effets de ce type sont envisageables. On peut par exemple imaginer de généraliser le principe de l'absorption saturée à deux faisceaux de pompage : le premier faisceau crée un " trou de population " dans le niveau du bas de la transition atomique utilisée (niveau $\mathrm{g}$ ) tandis que le deuxième faisceau modifie ce " trou de population ». Mais des effets plus spécifiques au pompage optique sont également possibles et ce sont ceux-là que nous nous proposons d'étudier dans cet article. Chaque faisceau est supposé suffisamment peu intense pour ne créer qu'une saturation optique négligeable, mais sa monochromaticité entraîne des effets de sélectivité en vitesses par le pompage optique. Le premier faisceau modifie les populations des différents sous-niveaux Zeeman du niveau g. Son effet peut être décrit en terme d'observables créées dans le niveau g : population $\left({ }^{1}\right)$, orientation, ou alignement. L'effet de saturation croisée est alors la modification par le deuxième faisceau de pompage des observables créées par le premier.

1.3 Exemple SIMPLE. - Plaçons-nous dans un cas simple de façon à présenter un effet possible de pompage optique croisé à deux faisceaux [14]. La polarisation des faisceaux $P_{1}$ et $P_{2}$ est choisie circulaire, tandis que le faisceau $\mathrm{D}$ mesure le dichroïsme circulaire de la vapeur contenue dans la cellule. Le signal obtenu est donc proportionnel à l'orientation du niveau étudié, selon l'axe $\mathrm{O} z$, colinéaire à $\mathrm{D}$.

Il est bien connu que l'action, sur un niveau donné, d'un faisceau lumineux polarisé circulairement, est double [3]. Les populations des sous-niveaux Zeeman sont modifiées différemment par le processus d'absorption et il en résulte une orientation $\langle\mathbf{J}\rangle_{0}$ dirigée selon la direction du faisceau de pompage. D'autre part, les sous-niveaux Zeeman sont déplacés différemment, et ces déplacements peuvent être décrits comme l'effet d'un champ magnétique fictif $\mathbf{B}_{\mathrm{f}}$ sur le niveau [13]. Comme $\langle\mathbf{J}\rangle_{0}, \mathbf{B}_{\mathrm{f}}$ est dirigé le long de la direction du faisceau lumineux et change de sens quand la polarisation de celui-ci passe de $\sigma^{+}$à $\sigma^{-}$.

Dans ces conditions, on voit qu'un effet de " pompage croisé " peut.résulter de l'action, sur l'orientation $\langle\mathbf{J}\rangle_{0}$ créée par un premier faisceau, du champ magnétique $B_{\mathrm{f}}$ associé à un deuxième faisceau. La figure 2 représente cette situation : le faisceau $P_{2}$ crée l'orientation $\langle\mathbf{J}\rangle_{0}$ dont l'évolution est modifiée par l'action du champ $B_{f}$ dû au faisceau $P_{1}$. On suppose que les atomes sont soumis à un champ magnétique $\mathbf{B}_{0}$, dirigé le long de $\mathrm{O} z$, et on admet dans un premier temps que le champ magnétique total vu par les atomes est suffisamment grand pour que leur orientation ait le temps de précesser un grand nombre de fois autour de lui pendant la durée de vie de l'orientation. Dans ce cas, la composante stationnaire de l'orientation perpendiculaire au champ magnétique est nulle. Un atome en résonance avec le faisceau $P_{2}$ seul voit son orientation $\langle\mathbf{J}\rangle$ précesser autour de $\mathbf{B}_{0}$, et la valeur stationnaire de l'orientation $\left\langle J_{z}\right\rangle$ détectée est simplement obtenue en projetant $\langle\mathbf{J}\rangle_{0}$ sur l'axe $\mathrm{O} z$ commun au champ magnétique et à la détection. Par contre, pour les atomes en résonance à la fois avec $P_{1}$ et $\mathbf{P}_{2}$, l'orientation $\langle\mathbf{J}\rangle$ précesse autour du champ magnétique total $\mathbf{B}_{0}+\mathbf{B}_{\mathrm{f}}$ résultant du champ appliqué et du champ fictif dû à $P_{1}$. L'orientation détectée $\left\langle J_{z}\right\rangle$ s'obtient donc en projetant $\langle\mathbf{J}\rangle_{0}$ d'abord sur la direction du champ magnétique total, puis sur $\mathrm{O} z$.

( $\left.{ }^{1}\right)$ Des modifications de population peuvent se produire si le niveau $\mathrm{g}$ se décompose, non seulement en sous-niveaux Zeeman, mais aussi en plusieurs niveaux de structure fine ou hyperfine (pompage hyperfin du sodium par exemple), ou encore si le niveau $g$ est un niveau métastable (trou de population introduit par le pompage dans le cas où le niveau e se désexcite vers d'autres niveaux). 


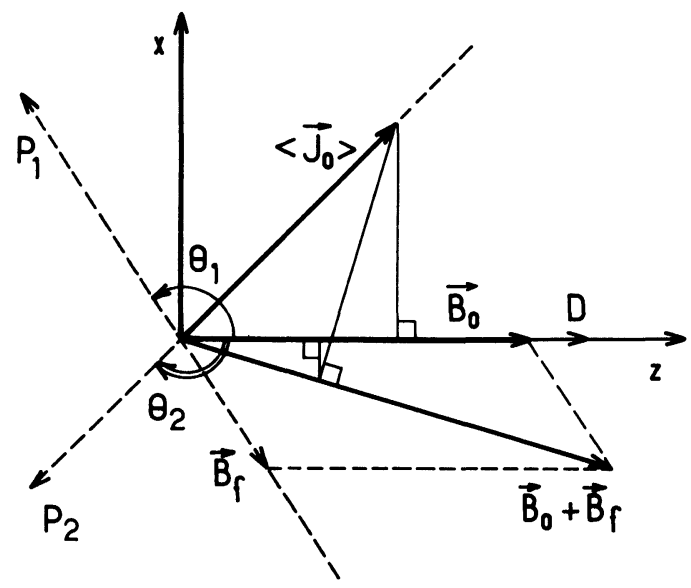

Fig. 2. - Exemple simple d'effet de pompage croisé : l'orientation $\langle\mathbf{J}\rangle_{0}$ créée par $\mathrm{P}_{2}$ précesse autour du champ total $\mathbf{B}_{0}+\mathbf{B}_{\mathrm{f}}$, où $\mathbf{B}_{\mathrm{f}}$ est le champ magnétique fictif associé au faisceau $\mathrm{P}_{1}$; le faisceau $\mathrm{D}$ détecte l'orientation le long de $\mathrm{O} z$ (voir le texte pour une description détaillée).

[Precession of the orientation $\langle\mathbf{J}\rangle_{0}$ created by $\mathrm{P}_{2}$ around the sum of a real field $\mathbf{B}_{0}$ and an effective field $\mathbf{B}_{\mathrm{f}}$ created by $P_{1}$. The detection beam $D$ provides a signal which is proportional to the projection on $\mathrm{O} z$ of the stationary value of the atomic orientation $\langle\mathbf{J}\rangle$.]

La valeur de $\left\langle J_{z}\right\rangle$ est simple à calculer, et l'on obtient un terme croisé à l'ordre le plus bas en $I_{1} I_{2}$ proportionnel à :

$$
J_{0}(\mathbf{v}) B_{\mathrm{f}}(\mathbf{v}) \sin \theta_{1} \sin \theta_{2} / \omega_{0}
$$

$J_{0}$ et $B_{\mathrm{f}}$ sont des fonctions de la vitesse $\mathbf{v}$ des atomes, respectivement proportionnelles à $I_{2}$ et $I_{1} ; \theta_{1}$ et $\theta_{2}$ sont les angles que font $\mathrm{P}_{1}$ et $\mathrm{P}_{2}$ avec $\mathrm{O} z ; \omega_{0}$ est la fréquence de Larmor du niveau $g$ dans le champ magnétique $\mathbf{B}_{0}$.

Dans le cas général où le champ total est quelconque, on ne peut plus négliger la composante transversale de l'orientation. Alors, un raisonnement géométrique simple que nous ne développerons pas ici (un calcul plus général est donné dans le $\S$ suivant) montre que le même terme croisé est proportionnel à :

$$
J_{0}(\mathbf{v}) B_{\mathrm{f}}(\mathbf{v}) \sin \theta_{1} \sin \theta_{2} \omega_{0}\left(\omega_{0}^{2}+\gamma^{2}\right)^{-1}
$$

$\gamma$ est le taux de relaxation de l'orientation dans le niveau $\mathrm{g}$.

Le signal d'orientation total détecté par le faisceau sonde, en se limitant au terme en $I_{1} I_{2}$, s'obtient en sommant cette expression, pondérée par la fonction de détection, sur toutes les vitesses possibles $\mathbf{v}$ des atomes, et en incluant l'effet inverse où l'orientation est créée par $P_{1}$ et le champ magnétique fictif est dû à $P_{2}$.

L'étude détaillée des caractéristiques du signal obtenu (dépendance en fréquence du laser, en champ magnétique, en angles $\theta_{1}$ et $\theta_{2}$ ), est faite dans la partie 2 dans le cas général, c'est-à-dire pour des polarisations quelconques des faisceaux de pompage et de détection.
2. Calcul général des effets de pompage optique à deux faisceaux. -2.1 HYPOTHÈSES DE CALCUL ET NOTATIONS. - On se propose ici d'étudier l'évolution d'un système atomique à deux niveaux $\mathrm{g}$ et $\mathrm{e}$, de moments cinétiques $J_{\mathrm{g}}$ et $J_{\mathrm{e}}$, sous l'action de deux ondes incidentes de vecteurs d'onde $\mathbf{k}_{1}$ et $\mathbf{k}_{2}$, issues du même laser dont la fréquence $\Omega_{\mathrm{V}} / 2 \pi$ est proche de la fréquence $\Omega_{0} / 2 \pi$ de la transition optique qui relie $\mathrm{g}$ et $\mathrm{e}$.

Le niveau e a une durée de vie $\tau_{\mathrm{e}}=\Gamma^{-1}$ beaucoup plus courte que celle du niveau g. Soit $\Gamma_{\mathrm{eg}}$ l'inverse de la durée de vie $\tau_{\text {eg }}$ de la cohérence optique associée à la transition $\mathrm{g} \leftrightarrow \mathrm{e}$. En l'absence de collisions déphasantes, on a :

$$
\Gamma_{\mathrm{eg}} \simeq \Gamma / 2 .
$$

On suppose que la largeur spectrale du laser est petite devant $\Gamma_{\mathrm{eg}}$. On peut donc, dans le référentiel du laboratoire, décrire les ondes incidentes comme des ondes planes classiques de polarisations respectives $\mathbf{e}_{\lambda_{1}}$ et $\mathbf{e}_{\lambda_{2}}$ et d'amplitudes $2 \varepsilon_{1}$ et $2 \varepsilon_{2}$ :

$$
\boldsymbol{E}_{1,2}(\mathbf{r}, t)=\mathbf{e}_{\lambda_{1,2}} \boldsymbol{\varepsilon}_{1,2} \exp \left[i\left(\mathbf{k}_{1,2} \cdot \mathbf{r}-\Omega_{\mathrm{L}} t\right)\right]+\text { c.c. }
$$

Un atome de vitesse $\mathbf{v}$ donnée dans le référentiel $\mathbf{d u}$ laboratoire voit, dans son référentiel, deux ondes de fréquences $\Omega_{1}$ et $\Omega_{2}$ décalées par effet Doppler.

$$
\Omega_{1,2}=\Omega_{\mathrm{L}}-\mathbf{k}_{1,2} \cdot \mathbf{v} .
$$

Le problème étudié se ramène donc, pour des atomes de vitesse $\mathbf{v}$ donnée, à celui du calcul de l'évolution du système atomique interagissant avec deux ondes de fréquences différentes.

Nous nous intéressons ici à des conditions où le système atomique, sous l'effet de l'interaction avec les champs électromagnétiques, évolue en "régime de pompage ". Dans le cas de l'excitation par un laser monomode, le temps de corrélation de cette interaction est long (il est égal à $\tau_{\mathrm{eg}}$ ), et l'évolution du système est en général du type " équations de Bloch ». Cependant, on sait [5], [15] qu'on obtient en fait un " régime de pompage " avec ce type de source à la limite des intensités faibles où :

$$
D_{\text {eg }} \mathcal{\hbar} \hbar \Gamma_{\text {eg }}
$$

où $\mathfrak{D}_{\mathrm{eg}}$ est de l'ordre de grandeur d'un élément de matrice d'une composante de $\boldsymbol{D}$ entre un sous-niveau de $g$ et un sous-niveau de e (D est le moment dipolaire électrique de l'atome) et $\varepsilon$ l'amplitude du champ électrique de l'onde. La condition (3) exprime que le temps caractéristique d'évolution des observables atomiques créées par le pompage est beaucoup plus grand que $\tau_{\mathrm{eg}}$. Nous supposerons que $\Gamma_{\mathrm{eg}}$ n'est pas beaucoup plus grand que $\Gamma$ : il est donc cohérent avec (3) de négliger les effets d'émission stimulée que pourraient induire les faisceaux.

Le système atomique évolue également sous l'action d'autres processus : relaxation, effet de champs appliqués, etc... La relaxation dans le niveau g peut être due 
à différentes causes : processus radiatifs, temps de transit fini des atomes dans les faisceaux et relaxation sur les parois, collisions entre atomes... Le taux de relaxation $\gamma$ dans ce niveau vérifie la condition :

$$
\gamma \ll \Gamma_{\text {eg }} .
$$

Dans ce cas, même si la condition (3) est vérifiée, les faisceaux de pompage peuvent avoir des effets importants sur les populations des différents sous-niveaux Zeeman du niveau métastable $\left(^{2}\right) \mathrm{g}$.

Le système atomique est soumis à un champ magnétique statique appliqué $\mathbf{B}_{0}$, dirigé selon $\mathrm{O} z$. L'amplitude $B_{0}$ de celui-ci est faible de sorte que les écarts Zeeman $\omega_{0}$ (resp. $\omega_{0}^{\prime}$ ) entre les sous-niveaux de $\mathrm{g}$ (resp. e) sont petits devant la largeur de la transition :

$$
\omega_{0}, \omega_{0}^{\prime} \ll \Gamma_{\text {eg }} .
$$

Par contre l'écart Zeeman $\omega_{0}$ dans le niveau métastable peut être comparable au taux de relaxation dans ce niveau :

$$
\omega_{0} \gtrsim \gamma
$$

2.2 EQUATIONS D'ÉVOLUTION DE L'OPÉRATEUR DENSITÉ. - 2.2.1 Rappel : pompage optique à un faisceau. - On appelle $\sigma$ l'opérateur densité du système atomique et $\sigma_{\mathrm{g}}$ (resp. $\sigma_{\mathrm{e}}$ ) sa restriction au sous-espace associé au niveau g (resp. e).

$$
\begin{aligned}
& \sigma_{\mathrm{g}}=P_{\mathrm{g}} \sigma P_{\mathrm{g}} \\
& \sigma_{\mathrm{e}}=P_{\mathrm{e}} \sigma P_{\mathrm{e}}
\end{aligned}
$$

$P_{\mathrm{g}}$ et $P_{\mathrm{e}}$ sont les projecteurs sur les niveaux $\mathrm{g}$ et $\mathrm{e}$.

Il est bien connu [3], [4] qu'en régime de pompage, la vitesse de variation de l'opérateur densité des atomes est la somme de trois termes :

$$
\frac{\mathrm{d} \sigma}{\mathrm{d} t}=\left.\frac{\mathrm{d} \sigma}{\mathrm{d} t}\right|_{1}+\left.\frac{\mathrm{d} \sigma}{\mathrm{d} t}\right|_{2}+\left.\frac{\mathrm{d} \sigma}{\mathrm{d} t}\right|_{3} .
$$

Le deuxième et le troisième terme rendent compte respectivement de l'émission spontanée et de l'évolution propre du système atomique sous l'action de diverses causes autres que l'interaction avec le rayonnement (relaxation par collisions, champ magnétique appliqué, etc...). Le premier terme décrit l'effet, sur le système atomique, de l'interaction avec les ondes lumineuses incidentes.

Pour une seule onde plane incidente quasi-monochromatique, de fréquence $\Omega$ et de polarisation $\mathbf{e}_{\lambda}$, le premier terme s'écrit :

$$
\left.\frac{\mathrm{d} \sigma_{\mathrm{g}}}{\mathrm{d} t}\right|_{1}=-\frac{\Gamma^{\prime}}{2}\left[A, \sigma_{\mathrm{g}}\right]_{+}-i \Delta E^{\prime}\left[A, \sigma_{\mathrm{g}}\right]
$$

( ${ }^{2}$ ) A partir de maintenant, on conviendra d'appeler « niveau métastable » le niveau g, car ceci correspond aux expériences que nous avons effectivement réalisées ; cependant, $g$ peut être un niveau fondamental ou un niveau radiatif, tant que l'inverse de sa durée de vie est petit devant $\Gamma_{\mathrm{eg}}$. et

$$
\left.\frac{\mathrm{d} \sigma_{\mathrm{e}}}{\mathrm{d} t}\right|_{1}=\Gamma^{\prime}\left(E \sigma_{\mathrm{g}}\right) .
$$

L'opérateur de pompage $A$ et l'opérateur d'excitation $E$ associés à l'onde incidente sont définis par :

$$
\begin{aligned}
A & =\left(\mathbf{e}_{\lambda}^{*} \cdot \mathbf{D}\right) P_{\mathrm{e}}\left(\mathbf{e}_{\lambda} \cdot \mathbf{D}\right) \\
\left(E \sigma_{\mathrm{g}}\right) & =\left(\mathbf{e}_{\lambda} \cdot \mathbf{D}\right) \sigma_{\mathrm{g}}\left(\mathbf{e}_{\lambda}^{*} \cdot \mathbf{D}\right)
\end{aligned}
$$

D, qui représente la partie angulaire de l'opérateur dipolaire électrique, est un opérateur tensoriel d'ordre 1 choisi de façon que $\left\langle J_{\mathrm{e}}\|\mathbf{D}\| J_{\mathrm{g}}\right\rangle=1 ; \Gamma^{\prime}$ et $\Delta E^{\prime}$ sont deux nombres réels proportionnels à l'intensité du faisceau incident, et ayant chacun la dimension de l'inverse d'un temps; ils valent respectivement :

$$
\left\{\begin{array}{l}
\Gamma^{\prime}=2 \omega_{1}^{2} \frac{\Gamma_{\mathrm{eg}}}{\Gamma_{\mathrm{eg}}^{2}+\left(\Omega-\Omega_{0}\right)^{2}} \\
\Delta E^{\prime}=\omega_{1}^{2} \frac{\Omega-\Omega_{0}}{\Gamma_{\mathrm{eg}}^{2}+\left(\Omega-\Omega_{0}\right)^{2}} .
\end{array}\right.
$$

On a posé

$$
\omega_{1}=\frac{|\mathfrak{D}| \mathcal{E}}{\hbar}
$$

avec

$$
\mathfrak{D}^{2}=\left|\left\langle J_{\mathrm{g}}\|\boldsymbol{D}\| J_{\mathrm{e}}\right\rangle\right|^{2} \text {. }
$$

Les fonctions $\Gamma^{\prime}$ et $\Delta E^{\prime}$ varient respectivement avec la fréquence de l'onde incidente comme une absorption et une dispersion de demi-largeur $\Gamma_{\mathrm{eg}}$.

Dans l'équation (6.a), le premier terme décrit le départ des atomes de l'état métastable lors du processus d'absorption (terme de dépopulation). Le coefficient $\Gamma^{\prime}$ est à un facteur près égal à $1 / T_{\mathrm{p}}$ où $T_{\mathrm{p}}$ est le temps de pompage associé au faisceau incident. L'opérateur $A$ n'est pas scalaire et le processus d'absorption est en général anisotrope (pour une polarisation donnée $\mathbf{e}_{\lambda}$, les sous-niveaux Zeeman de $\mathrm{g}$ peuvent être dépeuplés différemment). Le deuxième terme se présente comme un terme d'évolution propre sous l'action d'un hamiltonien effectif $\Delta E^{\prime} A$ [13]. Celui-ci a pour effet de déplacer les sous-niveaux. Zeeman de g d'une quantité qui dépend de la polarisation $e_{\lambda}$ et du sous-niveau Zeeman.

Les termes d'émission spontanée dans $\mathrm{d} \sigma / \mathrm{d} t$ s'écrivent :

$$
\begin{aligned}
& \left.\frac{\mathrm{d} \sigma_{\mathrm{g}}}{\mathrm{d} t}\right|_{2}=\mathcal{C}\left(\sigma_{\mathrm{e}}\right) \\
& \left.\frac{\mathrm{d} \sigma_{\mathrm{e}}}{\mathrm{d} t}\right|_{2}=-\Gamma \sigma_{\mathrm{e}} .
\end{aligned}
$$

L'opérateur $\mathcal{C}$, qui décrit le transfert par émission spontanée du niveau e vers le niveau g est un opérateur 
scalaire défini dans [3], [16]. Il est proportionnel à la probabilité par unité de temps $\Gamma_{\mathrm{r}}$ de retombée par émission spontanée du niveau e vers le niveau $\mathrm{g}$. Celle-ci n'est pas forcément égale à $\Gamma$, car le niveau e peut éventuellement se désexciter vers d'autres niveaux (soit radiativement, soit par collisions).

2.2.2 Cas de deux faisceaux. - Les équations (6) sont obtenues en supposant que les cohérences optiques ont une mémoire faible [condition (3)], ce qui conduit à des équations en « régime de pompage » qui peuvent être résolues à tous les ordres en $I$ (intensité du faisceau de pompage). Lorsqu'on a deux faisceaux de pompage, tous deux suffisamment faibles pour satisfaire à cette même condition, l'idée la plus naturelle est d'écrire directement que la variation temporelle de $\sigma$ est simplement la somme des variations imposées par chacun des faisceaux. Cette généralisation des équations (6) n'est en fait pas évidente, car la superposition des deux ondes incidentes pourrait donner lieu à des effets cohérents d'interférence qui sont ignorés dans le point de vue précédent. C'est pourquoi nous avons repris la résolution des équations générales d'évolution de l'opérateur densité $\sigma$ dans le cas de deux faisceaux d'intensités faibles (cf. appendice 1). On montre cependant que les termes d'interférence en $\varepsilon_{1} \varepsilon_{2}$ qui apparaissent dans $\sigma_{\mathrm{e}}$ et $\sigma_{\mathrm{g}}$ calculés au deuxième ordre en champ électrique apportent au signal croisé en $I_{1} I_{2}$ une contribution négligeable. Les seuls termes croisés importants sont ceux qui proviennent des termes en $\varepsilon_{1}^{2}$ et $\varepsilon_{2}^{2}$ dans $\sigma_{\mathrm{e}}$ et $\sigma_{\mathrm{g}}$ calculés au deuxième ordre en champ électrique : ils résultent en quelque sorte d'un premier cycle de pompage optique par un faisceau, suivi d'un deuxième cycle de pompage par l'autre.

Il est donc possible, pour calculer l'évolution des opérateurs densités $\sigma_{\mathrm{e}}$ et $\sigma_{\mathrm{g}}$, de faire un calcul en partant des relations suivantes où les effets des deux faisceaux ont simplement été ajoutés :

$$
\begin{aligned}
& \left.\frac{\mathrm{d} \sigma_{\mathrm{g}}}{\mathrm{d} t}\right|_{1}+\left.\frac{\mathrm{d} \sigma_{\mathrm{g}}}{\mathrm{d} t}\right|_{2}=-\frac{\Gamma_{1}^{\prime}}{2}\left[A_{1}, \sigma_{\mathrm{g}}\right]_{+}-i \Delta E_{1}^{\prime}\left[A_{1}, \sigma_{\mathrm{g}}\right]+i \operatorname{dem}(1 \rightarrow 2)+\mathcal{G}\left(\sigma_{\mathrm{e}}\right) \\
& \left.\frac{\mathrm{d} \sigma_{\mathrm{e}}}{\mathrm{d} t}\right|_{1}+\left.\frac{\mathrm{d} \sigma_{\mathrm{e}}}{\mathrm{d} t}\right|_{2}=\Gamma_{1}^{\prime}\left(E_{1} \sigma_{\mathrm{g}}\right)+\operatorname{idem}(1 \rightarrow 2)-\Gamma \sigma_{\mathrm{e}} .
\end{aligned}
$$

idem $(1 \rightarrow 2)$ représente la même expression que celle qui précède où l'on a remplacé partout l'indice 1 par 2 ( $A_{1}$ et $E_{1}$ sont les opérateurs de pompage et d'excitation associés au faisceau 1 ; la dépendance avec la fréquence $\Omega_{1}$ (resp. $\Omega_{2}$ ) est entièrement contenue dans $\Gamma_{1}^{\prime}$ et $\Delta E_{1}^{\prime}$ (resp. $\Gamma_{2}^{\prime}$ et $\left.\Delta E_{2}^{\prime}\right)$ ).

2.3 ETUDE DU RÉGIME STATIONNAIRE. - 2.3.1 Cas simple. - Pour calculer les termes de pompage croisés qui nous intéressent, qui sont du $2^{\mathrm{e}}$ ordre en intensité, il faut résoudre les équations $(8)$ par itération jusqu'à l'ordre 2. Nous allons dans un premier temps mener ce calcul dans un cas simple, de façon à mettre en évidence les effets de pompage croisés obtenus et à dégager leur signification physique : on ignore pour le moment les effets dus à la retombée par émission spontanée, et on suppose que le champ magnétique appliqué est nul. Le niveau évolue donc uniquement sous l'action des deux faisceaux incidents et de la relaxation. L'évolution globale de ${ }^{(n)} \sigma_{\mathrm{g}}$, opérateur densité $\sigma_{\mathrm{g}}$ calculé à l'ordre $n$ en intensité, est alors régie par l'équation :

$$
\begin{aligned}
& \frac{\mathrm{d}^{(n)} \sigma_{\mathrm{g}}}{\mathrm{d} t}=-\frac{\Gamma_{1}^{\prime}}{2}\left[A_{1},{ }^{(n-1)} \sigma_{\mathrm{g}}\right]_{+}- \\
& \quad-i \Delta E_{1}^{\prime}\left[A_{1},{ }^{(n-1)} \sigma_{\mathrm{g}}\right]+i \operatorname{dem}(1 \rightarrow 2)-\gamma^{(n)} \sigma_{\mathrm{g}} .
\end{aligned}
$$

Cette équation se résout simplement par itération, chaque ordre correspondant à un nombre donné de cycles de pompage optique. A l'ordre le plus bas en intensité (ordre 0), l'opérateur densité de g est isotrope :

$$
{ }^{(0)} \sigma_{\mathrm{g}}=\frac{P_{\mathrm{g}}}{2 J_{\mathrm{g}}+1} \text {. }
$$

En reportant cette valeur dans (9), les commutateurs s'annulent, et la solution de l'équation s'écrit :

$$
{ }^{(1)} \sigma_{\mathrm{g}}=-\frac{1}{\gamma\left(2 J_{\mathrm{g}}+1\right)}\left[\Gamma_{1}^{\prime} A_{1}+\Gamma_{2}^{\prime} A_{2}\right] \text {. }
$$

A l'ordre 1 en intensité, $\sigma_{\mathrm{g}}$ est une combinaison linéaire des opérateurs de pompage $A_{1}$ et $A_{2}$ où n'interviennent que les "termes de dépopulation ». Les effets de pompage croisé entre les deux faisceaux ne peuvent apparaître qu'à l'ordre suivant en reportant (10) dans (9). On obtient alors une équation où apparaissent des termes croisés en $I_{1} I_{2}$, en plus des termes en $I_{1}^{2}$ et $I_{2}^{2}$. Nous nous intéresserons ici uniquement aux termes croisés. Ils sont de deux sortes :

soit

$$
\frac{1}{2 \gamma\left(2 J_{\mathrm{g}}+1\right)} \Gamma_{1}^{\prime} \Gamma_{2}^{\prime}\left[A_{1}, A_{2}\right]_{+}+\operatorname{idem}(1 \rightarrow 2)
$$

soit

$$
\frac{i}{\gamma\left(2 J_{\mathrm{g}}+1\right)} \Delta E_{1}^{\prime} \Gamma_{2}^{\prime}\left[A_{1}, A_{2}\right]+i \operatorname{dem}(1 \rightarrow 2) .
$$

Ces termes croisés peuvent être appelés respectivement termes de « dépopulation-dépopulation » et termes de " dépopulation-déplacement ». Dans ce cas particulier, la structure des termes de « pompage croisé » est donc particulièrement simple.

2.3.2 Cas général. Décomposition sur une base d'opérateurs tensoriels irréductibles. - Si l'on tient compte de l'émission spontanée, et qu'on se place en présence d'un champ magnétique appliqué $\mathbf{B}_{0}$, il faut 
reprendre les équations (8) en ajoutant dans chacune un terme d'évolution propre :

$$
\begin{aligned}
& \left.\frac{\mathrm{d}^{(n)} \sigma_{\mathrm{g}}}{\mathrm{d} t}\right|_{3}=-i\left[H_{\mathrm{g}},{ }^{(n)} \sigma_{\mathrm{g}}\right]-\gamma^{(n)} \sigma_{\mathrm{g}} \\
& \left.\frac{\mathrm{d}^{(n)} \sigma_{\mathrm{e}}}{\mathrm{d} t}\right|_{3}=-i\left[H_{\mathrm{e}},{ }^{(n)} \sigma_{\mathrm{e}}\right]-\Gamma^{(n)} \sigma_{\mathrm{e}} .
\end{aligned}
$$

Supposons que $\mathbf{B}_{0}$ est dirigé selon $\mathrm{O} z$. Les hamiltoniens qui lui sont associés s'écrivent :

$$
\begin{aligned}
& H_{\mathrm{g}}=\omega_{0} \boldsymbol{P}_{\mathrm{g}} J_{\mathrm{z}} \boldsymbol{P}_{\mathrm{g}} \\
& \boldsymbol{H}_{\mathrm{e}}=\omega_{0}^{\prime} \boldsymbol{P}_{\mathrm{e}} J_{z} P_{\mathrm{e}}
\end{aligned}
$$

$P_{\mathrm{g}} J_{z} P_{\mathrm{g}}\left(\right.$ resp. $P_{\mathrm{e}} J_{z} P_{\mathrm{e}}$ ) est l'orientation le long de $\mathrm{O} z$ dans le niveau $g$ (resp. e).

Nous supposerons par la suite que l'amplitude du champ magnétique appliqué $\mathbf{B}_{0}$ est telle que $\omega_{0}^{\prime}$ soit très petit devant $\Gamma$. Nous négligerons donc l'action du champ magnétique sur les observables du niveau excité ; cette approximation revient à faire $H_{\mathrm{e}}=0$.

Pour résoudre les équations d'évolution globales de $\sigma_{\mathrm{g}}$ et $\sigma_{\mathrm{e}}$ obtenues alors, il est commode de décomposer chaque opérateur densité sur une base d'opérateurs tensoriels irréductibles. Les opérateurs densités $\sigma_{\mathrm{e}}$ et $\sigma_{\mathrm{g}}$ s'écrivent :

$$
\left.\begin{array}{l}
\sigma_{\mathrm{g}}=\sum_{k, q} \sigma_{\mathrm{g} q}^{(k)} T_{q}^{(k)}\left(J_{\mathrm{g}}\right) \\
\sigma_{\mathrm{e}}=\sum_{k, q} \sigma_{\mathrm{eq}}^{(k)} T_{q}^{(k)}\left(J_{\mathrm{e}}\right) \cdot
\end{array}\right\}
$$

$T_{q}^{(k)}(J)$ est l'opérateur tensoriel irréductible normé agissant à l'intérieur du niveau de moment cinétique $J$ [17]. Son sens physique pour différentes valeurs de $k$ et $q$ est bien connu [18], [19]; en particulier :

$\left\langle T_{0}^{(0)}\right\rangle$ est proportionnel à la population totale du niveau considéré ;

$\left\langle T_{0}^{(1)}\right\rangle$ est proportionnel à l'orientation $\left\langle J_{z}\right\rangle$ dans ce niveau, le long de l'axe $\mathrm{O} z$;

$\left\langle T_{0}^{(2)}\right\rangle$ et $\left\langle T_{ \pm 2}^{(2)}\right\rangle$ sont, à un facteur près, les composantes longitudinales et transversales de l'alignement dans le niveau.

L'intérêt de cette décomposition est que l'effet du champ magnétique dans le niveau $\mathrm{g}$ s'exprime de façon simple. En effet comme :

$$
\left[P_{\mathrm{g}} J_{z} P_{\mathrm{g}}, T_{q}^{(k)}\left(J_{\mathrm{g}}\right)\right]=q T_{q}^{(k)}\left(J_{\mathrm{g}}\right) \text {. }
$$

Le terme d'évolution propre s'écrit :

$$
\left.\frac{\mathrm{d}}{\mathrm{d} t} \sigma_{\mathrm{g}}\right|_{3}=-\sum_{k q}\left(\gamma+i q \omega_{0}\right) \sigma_{\mathrm{g} q}^{(k)} T_{q}^{(k)}\left(J_{\mathrm{g}}\right) .
$$

Les opérateurs de pompage et d'excitation peuvent être décomposés de la même façon, en posant :

$$
\begin{aligned}
& A_{1,2}=\sum_{k, q} A_{1,2 q}{ }^{(k)} T_{q}^{(k)}\left(J_{\mathrm{g}}\right) \\
& E_{1,2}=\sum_{k, q} E_{1,2 q}(k) T_{q}^{(k)}\left(J_{\mathrm{e}}\right) .
\end{aligned}
$$

L'opérateur $E_{1,2}$ est défini par :

$$
E_{1,2}=\left(E_{1,2} P_{\mathrm{g}}\right) \text {. }
$$

Les composantes $A_{1,2 q}{ }^{(k)}$ et $E_{1,2 q}{ }^{(k)}$ dépendent des polarisations $\mathbf{e}_{\lambda_{1}}$ et $\mathbf{e}_{\lambda_{2}}$. Pour une excitation lumineuse statique, elles sont indépendantes du temps, et en régime stationnaire les composantes $\sigma_{\mathrm{gq}}^{(k)}$ et $\sigma_{\mathrm{eq}}^{(k)}$ également.

On peut décomposer le vecteur polarisation $\mathbf{e}_{\lambda}$ sur une base standard définie par [17] :

$$
\left\{\begin{array}{l}
\mathbf{e}_{ \pm 1}=\mp \frac{\left(\mathbf{e}_{x} \pm i \mathbf{e}_{y}\right)}{\sqrt{2}} \\
\mathbf{e}_{0}=\mathbf{e}_{z} \\
\mathbf{e}_{\lambda}=\sum_{p}(-1)^{p} \alpha_{-p} \mathbf{e}_{p} .
\end{array}\right.
$$

Les coefficients $\alpha_{p}$ sont les composantes standards de $\mathbf{e}_{\lambda}$. Le référentiel Oxyz est orthonormé et fixe par rapport au laboratoire; l'axe $\mathrm{O} z$ du champ magnétique a été choisi comme axe de quantification.

La partie angulaire $\mathbf{D}$ de l'opérateur dipolaire électrique peut être décomposée de façon analogue :

$$
\mathbf{D}=\sum_{p}(-1)^{p} D_{-p} \mathbf{e}_{p} .
$$

Avec ces notations, les opérateurs de pompage et d'excitation s'écrivent [18] :

$$
\begin{aligned}
A & =\sum_{p p^{\prime}}(-1)^{p} \alpha_{p^{\prime}}^{*} \alpha_{-p} D_{p^{\prime}} P_{\mathrm{e}} D_{p} \\
E & =\sum_{p p^{\prime}}(-1)^{p} \alpha_{p^{\prime}}^{*} \alpha_{-p} D_{p} P_{\mathrm{g}} D_{p^{\prime}}
\end{aligned}
$$

On peut montrer que leurs composantes sont de la forme :

$$
\left.\begin{array}{l}
A_{q}^{(k)}=(-1)^{k} B_{k}\left(J_{\mathrm{g}}, J_{\mathrm{e}}\right) \varphi_{q}^{(k)}\left(\mathbf{e}_{\lambda}\right) \\
E_{q}^{(k)}=B_{k}\left(J_{\mathrm{e}}, J_{\mathrm{g}}\right) \varphi_{q}^{(k)}\left(\mathbf{e}_{\lambda}\right)
\end{array}\right\}
$$

avec

$$
B_{k}\left(J_{1}, J_{2}\right)=(-1)^{J_{1}+J_{2}+k+1}\left\{\begin{array}{ccc}
1 & 1 & k \\
J_{1} & J_{1} & J_{2}
\end{array}\right\}
$$

et

$$
\varphi_{q}^{(k)}\left(\mathbf{e}_{\lambda}\right)=\sum_{p p^{\prime}}(-1)^{p+1} \alpha_{p^{\prime}}^{*} \alpha_{-p}\left\langle 11 p p^{\prime} \mid k q\right\rangle .
$$

Les coefficients $\varphi_{q}^{(k)}\left(\mathbf{e}_{\lambda}\right)$ sont identiques à ceux définis dans [20]. Leurs valeurs sont données dans le tableau I dans le cas d'un faisceau de polarisation circulaire ou linéaire. On a supposé que le faisceau se propage dans le plan orienté $z \mathrm{O} x$ dans une direction qui fait l'angle algébrique $\theta$ avec $\mathrm{O} z$. 
Tableau I.

\begin{tabular}{|c|c|c|c|c|c|c|}
\hline Polarisations & $\varphi_{0}^{(0)}$ & $\varphi_{0}^{(1)}$ & $\varphi_{ \pm 1}^{(1)}$ & $\varphi_{0}^{(2)}$ & $\varphi_{ \pm 1}^{(2)}$ & $\varphi_{ \pm 2}^{(2)}$ \\
\hline$\sigma^{+}$ & $+\frac{1}{\sqrt{3}}$ & $+\frac{\cos \theta}{\sqrt{2}}$ & $\mp \frac{\sin \theta}{2}$ & $-\frac{3 \sin ^{2} \theta-2}{2 \sqrt{6}}$ & $\mp \frac{\sin \theta \cos \theta}{2}$ & $+\frac{\sin ^{2} \theta}{4}$ \\
\hline$\sigma^{-}$ & $+\frac{1}{\sqrt{3}}$ & $-\frac{\cos \theta}{\sqrt{2}}$ & $\pm \frac{\sin \theta}{2}$ & $-\frac{3 \sin ^{2} \theta-2}{2 \sqrt{6}}$ & $\mp \frac{\sin \theta \cos \theta}{2}$ & $+\frac{\sin ^{2} \theta}{4}$ \\
\hline $\begin{array}{c}\uparrow \text { linéaire } \\
\text { parallèle à Oy }\end{array}$ & $+\frac{1}{\sqrt{3}}$ & 0 & 0 & $+\frac{1}{\sqrt{6}}$ & 0 & $+\frac{1}{2}$ \\
\hline $\begin{array}{c}\leftrightarrow \text { linéaire dans le } \\
\text { plan } z \mathrm{O} x\end{array}$ & $+\frac{1}{\sqrt{3}}$ & 0 & 0 & $-\frac{3 \sin ^{2} \theta-1}{\sqrt{6}}$ & $\mp \sin \theta \cos \theta$ & $-\frac{\cos ^{2} \theta}{2}$ \\
\hline
\end{tabular}

2.3.3 Résolution des équations par itération. Effets de pompage croisé. - A l'ordre 0 en intensité, on a toujours :

$$
{ }^{(0)} \sigma_{\mathrm{g}}=\frac{P_{\mathrm{g}}}{2 J_{\mathrm{g}}+1} \text {. }
$$

A l'ordre 1 en intensité, l'évolution de ${ }^{(1)} \sigma_{\mathrm{e}}$ est donc régie par l'équation :

$$
\frac{\mathrm{d}^{(1)} \sigma_{\mathrm{e}}}{\mathrm{d} t}=\frac{\Gamma_{1}^{\prime}}{2 J_{\mathrm{g}}+1} \sum_{k_{1}, q_{1}} E_{1 q_{1}}^{\left(k_{1}\right)} T_{q_{1}}^{\left(k_{1}\right)}\left(J_{\mathrm{e}}\right)+\operatorname{idem}(1 \rightarrow 2)-\Gamma^{(1)} \sigma_{\mathrm{e}} .
$$

La solution de cette équation s'écrit :

$$
{ }^{(1)} \sigma_{\mathrm{e}}=\frac{1}{\Gamma} \frac{\Gamma_{1}^{\prime}}{2 J_{\mathrm{g}}+1} \sum_{k_{1}, q_{1}} E_{1 q_{1}}^{\left(k_{1}\right)} T_{q_{1}}^{\left(k_{1}\right)}\left(J_{\mathrm{e}}\right)+\operatorname{idem}(1 \rightarrow 2) .
$$

La matrice densité ${ }^{(1)} \sigma_{\mathrm{g}}$ du niveau métastable évolue sous l'effet de quatre causes (interaction avec les faisceaux incidents, retombée par émission spontanée $\left({ }^{3}\right)$, relaxation et effet du champ magnétique statique) :

$$
\begin{aligned}
& \left.\frac{\mathrm{d}^{(1)} \sigma_{\mathrm{g}}}{\mathrm{d} t}\right|_{1}=-\frac{\Gamma_{1}^{\prime}}{2 J_{\mathrm{g}}+1} \sum_{k_{1}, q_{1}} A_{1 q_{1}}^{\left(k_{1}\right)} T_{q_{1}}^{\left(k_{1}\right)}\left(J_{\mathrm{g}}\right)+i \operatorname{dem}(1 \rightarrow 2) \\
& \left.\frac{\mathrm{d}^{(1)} \sigma_{\mathrm{g}}}{\mathrm{d} t}\right|_{2}=\frac{\Gamma_{\mathrm{r}}}{\Gamma} \frac{\Gamma_{1}^{\prime}}{2 J_{\mathrm{g}}+1}\left(2 J_{\mathrm{e}}+1\right) \sum_{k_{1}, q_{1}}(-1)^{J_{\mathrm{e}}+J_{\mathrm{g}}+k_{1}+1}\left\{\begin{array}{ccc}
J_{\mathrm{e}} & J_{\mathrm{e}} & k_{1} \\
J_{\mathrm{g}} & J_{\mathrm{g}} & 1
\end{array}\right\} E_{1 q_{1}}^{\left(k_{1}\right)} T_{q_{1}}^{\left(k_{1}\right)}\left(J_{\mathrm{g}}\right)+\operatorname{idem}(1 \rightarrow 2) \\
& \left.\frac{\mathrm{d}^{(1)} \sigma_{\mathrm{g}}}{\mathrm{d} t}\right|_{3}=-\sum_{k_{1}, q_{1}}\left(\gamma+i q_{1} \omega_{0}\right)^{(1)} \sigma_{\mathrm{g}_{1}}^{\left(k_{1}\right)} T_{q_{1}}^{\left(k_{1}\right)}\left(J_{\mathrm{g}}\right) .
\end{aligned}
$$

Les deux premiers termes se regroupent simplement, et l'intégration de l'équation d'évolution de ${ }^{(1)} \sigma_{\mathrm{g}}$ donne :

$$
{ }^{(1)} \sigma_{\mathrm{g}}=\frac{\Gamma_{1}^{\prime}}{2 J_{\mathrm{g}}+1} \sum_{k_{1}, q_{1}} \mathcal{B}_{k_{1}}\left(J_{\mathrm{g}}, J_{\mathrm{e}}\right) \frac{\varphi_{q_{1}}^{\left(k_{1}\right)}\left(\mathbf{e}_{\lambda_{1}}\right) T_{q_{1}}^{\left(k_{1}\right)}\left(J_{\mathrm{g}}\right)}{i q_{1} \omega_{0}+\gamma}+\operatorname{idem}(1 \rightarrow 2)
$$

on a posé :

$$
\mathcal{B}_{k_{1}}\left(J_{\mathrm{g}}, J_{\mathrm{e}}\right)=(-1)^{k_{1}+1} B_{k_{1}}\left(J_{\mathrm{g}}, J_{\mathrm{e}}\right)+(-1)^{J_{\mathrm{e}}+J_{\mathrm{g}}+k_{1}+1}\left(2 J_{\mathrm{e}}+1\right) \frac{\Gamma_{\mathrm{r}}}{\Gamma}\left\{\begin{array}{ccc}
J_{\mathrm{e}} & J_{\mathrm{e}} & k_{1} \\
J_{\mathrm{g}} & J_{\mathrm{g}} & 1
\end{array}\right\} B_{k_{1}}\left(J_{\mathrm{e}}, J_{\mathrm{g}}\right)
$$

Dans cette expression qui décrit l'effet d'un cycle de pompage par le faisceau 1, le premier terme correspond à la dépopulation, et le deuxième à la retombée.

A l'ordre 2 en intensité, l'équation d'évolution de ${ }^{(2)} \sigma_{\mathrm{e}}$ s'écrit en utilisant l'expression (21). Nous nous

$\left({ }^{3}\right)$ La deuxième expression peut être déduite de (20) en utilisant les résultats de M. Ducloy [16]. 
contenterons ici d'écrire les termes de pompage croisés liés à l'interaction du système atomique d'abord avec un faisceau ( $1^{\mathrm{er}}$ cycle de pompage optique) puis avec l'autre ( $2^{\mathrm{e}}$ cycle) :

$$
\frac{\mathrm{d}^{(2)} \sigma_{\mathrm{e}}}{\mathrm{d} t}=\frac{\Gamma_{1}^{\prime} \Gamma_{2}^{\prime}}{2 J_{\mathrm{g}}+1} \sum_{k_{1}, q_{1}} \frac{\Re_{k_{1}}\left(J_{\mathrm{g}}, J_{\mathrm{e}}\right) \varphi_{q_{1}}^{\left(k_{1}\right)}\left(\mathbf{e}_{i_{1}}\right)}{i q_{1} \omega_{0}+\gamma}\left(E_{2} T_{q_{1}}^{\left(k_{1}\right)}\left(j_{\mathrm{g}}\right)\right)+i d e m(1 \rightarrow 2)-\Gamma^{(2)} \sigma_{\mathrm{e}} .
$$

L'opérateur $\left(E_{2} T_{q_{1}}^{\left(k_{1}\right)}\left(J_{\mathrm{g}}\right)\right)$ peut être décomposé sur la base des $T_{Q}^{(K)}\left(J_{\mathrm{e}}\right)$; ses composantes dans cette base sont données par A. Omont [18] et valent :

$$
\sum_{k_{2}, q_{2}} \varphi_{q_{2}}^{\left(k_{2}\right)}\left(\mathbf{e}_{\lambda_{2}}\right) \sqrt{\left(2 k_{1}+1\right)\left(2 k_{2}+1\right)}\left\{\begin{array}{lll}
J_{\mathrm{e}} & J_{\mathrm{g}} & 1 \\
J_{\mathrm{e}} & J_{\mathrm{g}} & 1 \\
K & k_{1} & k_{2}
\end{array}\right\}\left\langle k_{1} k_{2} q_{1} q_{2} \mid K Q\right\rangle .
$$

Les composantes de l'opérateur ${ }^{(2)} \sigma_{\mathrm{e}}$ sont donc de la forme :

$$
\begin{aligned}
{ }^{(2)} \sigma_{\mathrm{eQ}}^{(K)}=\frac{\Gamma_{1}^{\prime} \Gamma_{2}^{\prime}}{\Gamma\left(2 J_{\mathrm{g}}+1\right)} \sum_{\substack{k_{1}, k_{2} \\
q_{1}, q_{2}}} \frac{\mathcal{B}_{k_{1}}\left(J_{\mathrm{g}}, J_{\mathrm{e}}\right) \varphi_{q_{1}}^{\left(k_{1}\right)}\left(\mathbf{e}_{\lambda_{1}}\right) \varphi_{q_{2}}^{\left(k_{2}\right)}\left(\mathbf{e}_{\lambda_{2}}\right)}{i q_{1} \omega_{0}+\gamma} \sqrt{\left(2 k_{1}+1\right)\left(2 k_{2}+1\right)} \times \\
\times\left\{\begin{array}{ccc}
J_{\mathrm{e}} & J_{\mathrm{g}} & 1 \\
J_{\mathrm{e}} & J_{\mathrm{g}} & 1 \\
K & k_{1} & k_{2}
\end{array}\right\}\left\langle k_{1} k_{2} q_{1} q_{2} \mid K Q\right\rangle+\operatorname{idem}(1 \rightarrow 2) .
\end{aligned}
$$

Comme ceux de $\frac{\mathrm{d}^{(1)} \sigma_{\mathrm{g}}}{\mathrm{d} t}$, les termes apparaissant dans $\frac{\mathrm{d}^{(2)} \sigma_{\mathrm{g}}}{\mathrm{d} t}$ sont de quatre sortes; si on se limite aux termes croisés, ceux qui décrivent l'interaction avec le faisceau lumineux contiennent soit des anticommutateurs (termes en $\Gamma_{1}^{\prime} \Gamma_{2}^{\prime}$ ) soit des commutateurs (termes en $\Gamma_{1}^{\prime} \Delta E_{2}^{\prime}$ et $\Gamma_{2}^{\prime} \Delta E_{1}^{\prime}$ ):

$$
\begin{aligned}
& \left.\frac{\mathrm{d}^{(2)} \sigma_{\mathrm{g}}}{\mathrm{d} t}\right|_{1}=-\frac{\Gamma_{1}^{\prime} \Gamma_{2}^{\prime}}{2\left(2 J_{\mathrm{g}}+1\right)} \sum_{\substack{k_{1}, q_{1} \\
k_{2}, q_{2}}}(-1)^{k_{2}} B_{k_{2}}\left(J_{\mathrm{g}}, J_{\mathrm{e}}\right) \mathcal{B}_{k_{1}}\left(J_{\mathrm{g}}, J_{\mathrm{e}}\right) \frac{\varphi_{q_{2}}^{\left(k_{2}\right)}\left(\mathbf{e}_{\lambda_{2}}\right) \varphi_{q_{1}}^{\left(k_{1}\right)}\left(\mathbf{e}_{\lambda_{1}}\right)}{i q_{1} \omega_{0}+\gamma}\left[T_{q_{1}}^{\left(k_{1}\right)}\left(J_{\mathrm{g}}\right), T_{q_{2}}^{\left(k_{2}\right)}\left(J_{\mathrm{g}}\right)\right]_{+} \\
& +\frac{i \Gamma_{1}^{\prime} \Delta E_{2}^{\prime}}{2 J_{\mathrm{g}}+1} \sum_{\substack{k_{1}, q_{1} \\
k_{2}, q_{2}}}(-1)^{k_{2}} B_{k_{2}}\left(J_{\mathrm{g}}, J_{\mathrm{e}}\right) \mathcal{B}_{k_{1}}\left(J_{\mathrm{g}}, J_{\mathrm{e}}\right) \frac{\varphi_{q_{2}}^{\left(k_{2}\right)}\left(\mathbf{e}_{\lambda_{2}}\right) \varphi_{q_{1}}^{\left(k_{1}\right)}\left(\mathbf{e}_{\lambda_{1}}\right)}{i q_{1} \omega_{0}+\gamma}\left[T_{q_{1}}^{\left(k_{1}\right)}\left(J_{\mathrm{g}}\right), T_{q_{2}}^{\left(k_{2}\right)}\left(J_{\mathrm{g}}\right)\right]+\operatorname{idem}(1 \rightarrow 2) .
\end{aligned}
$$

Ces deux termes sont la généralisation des expressions (11) et (12) en présence d'un champ magnétique.

A partir des $\left(2 k_{1}+1\right)\left(2 k_{2}+1\right)$ opérateurs $T_{q_{1}}^{\left(k_{1}\right)}\left(J_{\mathrm{g}}\right) T_{q_{2}}^{\left(k_{2}\right)}\left(J_{\mathrm{g}}\right)$, on peut construire un ensemble d'opérateurs tensoriels irréductibles $V_{Q}^{(K)}\left(J_{\mathrm{g}}\right)$ d'ordre $K\left(\left|k_{1}-k_{2}^{q_{1}}\right| \leqslant K \leqslant k_{1}+k_{2}\right)[21]$ :

$$
V_{Q}^{(K)}\left(J_{\mathrm{g}}\right)=\sum_{\substack{k_{1}, q_{1} \\ k_{2}, q_{2}}}\left\langle k_{1} k_{2} q_{1} q_{2} \mid K Q\right\rangle T_{q_{1}}^{\left(k_{1}\right)}\left(J_{\mathrm{g}}\right) T_{q_{2}}^{\left(k_{2}\right)}\left(J_{\mathrm{g}}\right)
$$

L'élément de matrice réduit de $V^{(K)}$ s'obtient en utilisant la formule (7.1.1) de [22] :

$$
\left\langle J_{\mathrm{g}}\left\|V^{K}\right\| J_{\mathrm{g}}\right\rangle=(-1)^{K+2 J_{\mathrm{g}}} \sqrt{\left(2 k_{1}+1\right)\left(2 k_{2}+1\right)(2 K+1)}\left\{\begin{array}{lll}
k_{1} & k_{2} & K \\
J_{\mathrm{g}} & J_{\mathrm{g}} & J_{\mathrm{g}}
\end{array}\right\} .
$$

Avec ces notations, les produits $T_{q_{1}}^{\left(k_{1}\right)} T_{q_{2}}^{\left(k_{2}\right)}$ peuvent s'écrire :

$$
T_{q_{1}}^{\left(k_{1}\right)}\left(J_{\mathrm{g}}\right) T_{q_{2}}^{\left(k_{2}\right)}\left(J_{\mathrm{g}}\right)=\sum_{K, Q}\left\langle k_{1} k_{2} q_{1} q_{2} \mid K Q\right\rangle \frac{\left\langle J_{\mathrm{g}}\left\|V^{(K)}\right\| J_{\mathrm{g}}\right\rangle}{\sqrt{2 K+1}} T_{Q}^{(K)}\left(J_{\mathrm{g}}\right)
$$

où $T_{Q}^{(K)}\left(J_{\mathrm{g}}\right)$ est l'opérateur tensoriel irréductible normé d'ordre $K$ agissant dans le niveau $\mathrm{g}$.

On déduit simplement, des relations de symétrie des coefficients de Clebsch-Gordan, que :

$$
\left[T_{q_{1}}^{\left(k_{1}\right)}\left(J_{\mathrm{g}}\right), T_{q_{2}}^{\left(k_{2}\right)}\left(J_{\mathrm{g}}\right)\right]_{ \pm}=\sum_{K, Q} \frac{\left\langle J_{\mathrm{g}}\left\|V^{(K)}\right\| J_{\mathrm{e}}\right\rangle}{\sqrt{2 K+1}}\left\langle k_{1} k_{2} q_{1} q_{2} \mid K Q\right\rangle\left[1 \pm(-1)^{k_{1}+k_{2}+K}\right] T_{Q}^{(K)}\left(J_{\mathrm{g}}\right) .
$$

Pour être non nuls, les termes apparaissant dans (24) doivent donc vérifier :

- soit la condition $k_{1}+k_{2}+K$ pair; il s'agit alors de termes en $\Gamma_{1}^{\prime} \Gamma_{2}^{\prime}$;

- soit la condition $k_{1}+k_{2}+K$ impair ; il s'agit alors de termes en $\Gamma_{1}^{\prime} \Delta E_{2}^{\prime}$ ou $\Gamma_{2}^{\prime} \Delta E_{1}^{\prime}$. 
Remarquons que le coefficient $9 j$ qui figure dans l'expression (23) est nul si $k_{1}+k_{2}+K$ est impair : ceci est naturel, car les propriétés de symétrie de l'opérateur $\sigma_{\mathrm{e}}$ créé par un processus d'absorption sont les mêmes que celles de l'opérateur $\sigma_{\mathrm{g}}$ résultant de la dépopulation par ce processus.

A ces termes, il faut ajouter ceux qui proviennent de la retombée par émission spontanée ;

$$
\begin{aligned}
\frac{\mathrm{d}^{(2)} \sigma_{\mathrm{g}}}{\mathrm{d} t} & =\frac{\Gamma_{\mathrm{r}}}{\Gamma} \frac{\Gamma_{1}^{\prime} \Gamma_{2}^{\prime}}{2 J_{\mathrm{g}}+1}\left(2 J_{\mathrm{e}}+1\right) \sum_{\substack{k_{1}, k_{2} \\
q_{1}, q_{2}}} \sum_{K, Q} \frac{\mathcal{B}_{k_{1}}\left(J_{\mathrm{g}}, J_{\mathrm{e}}\right) \varphi_{q_{1}}^{\left(k_{1}\right)}\left(\mathbf{e}_{\lambda_{1}}\right) \varphi_{q_{2}}^{\left(k_{2}\right)}\left(\mathbf{e}_{\lambda_{2}}\right)}{i q_{1} \omega_{0}+\gamma}(-1)^{J_{\mathrm{e}}+J_{\mathrm{g}}+K+1} \times \\
& \times \sqrt{\left(2 k_{1}+1\right)\left(2 k_{2}+1\right)}\left\{\begin{array}{lll}
J_{\mathrm{e}} & J_{\mathrm{g}} & 1 \\
J_{\mathrm{e}} & J_{\mathrm{g}} & 1 \\
K & k_{1} & k_{2}
\end{array}\right\}\left\{\begin{array}{lll}
J_{\mathrm{e}} & J_{\mathrm{e}} & K \\
J_{\mathrm{g}} & J_{\mathrm{g}} & 1
\end{array}\right\}\left\langle k_{1} k_{2} q_{1} q_{2} \mid K Q\right\rangle T_{Q}^{(K)}\left(J_{\mathrm{g}}\right)+\text { idem }(1 \rightarrow 2) .
\end{aligned}
$$

$\mathrm{Au}$ total, les termes sources dans l'évolution de ${ }^{(2)} \sigma_{\mathrm{g}}$ sont de deux sortes :

- des termes de " pompage-pompage » en $\Gamma_{1}^{\prime} \Gamma_{2}^{\prime}$ où l'on a regroupé les effets dus à la dépopulation et à la retombée; ils correspondent à $k_{1}+k_{2}+K$ pair ;

- des termes de " pompage-déplacement » en $\Gamma_{1}^{\prime} \Delta E_{2}^{\prime}$ et $\Gamma_{2}^{\prime} \Delta E_{1}^{\prime}$ où la retombée lors du $2^{\mathrm{e}}$ cycle de pompage n'intervient pas; ils correspondent à $k_{1}+k_{2}+K$ impair.

En regroupant les différents termes, l'équation d'évolution de ${ }^{(2)} \sigma_{\mathrm{g}}$ se résout simplement; dans la composante ${ }^{(2)} \sigma_{\mathrm{gQ}}^{(K)} \mathrm{de}^{(2)} \sigma_{\mathrm{g}}$ sur la base des $T_{Q}^{(K)}\left(J_{\mathrm{g}}\right)$, apparaissent deux sortes de termes croisés :

- des termes de "pompage-pompage» $\left(k_{1}+k_{2}+K\right.$ pair $)$ :

$$
\begin{aligned}
& \frac{\Gamma_{1}^{\prime} \Gamma_{2}^{\prime}}{2 J_{\mathrm{g}}+1} \frac{1}{i Q \omega_{0}}+\sum_{\substack{k_{1}, q_{1} \\
k_{2}, q_{2}}}\left\langle k_{1} k_{2} q_{1} q_{2} \mid K Q\right\rangle \frac{\varphi_{q_{1}}^{\left(k_{1}\right)}\left(\mathbf{e}_{\lambda_{1}}\right) \varphi_{q_{2}}^{\left(k_{2}\right)}\left(\mathbf{e}_{\lambda_{2}}\right)}{i q_{1} \omega_{0}+\gamma} \times \\
& \times\left[\mathcal { B } _ { k _ { 1 } } ( J _ { \mathrm { g } } , J _ { \mathrm { e } } ) \left(\frac{\left\langle J_{\mathrm{g}}\left\|V^{(K)}\right\| J_{\mathrm{g}}\right\rangle}{\sqrt{2 K+1}}(-1)^{k_{2}+1} B_{k_{2}}\left(J_{\mathrm{g}}, J_{\mathrm{e}}\right)+\frac{\Gamma_{\mathrm{r}}}{\Gamma}(-1)^{J_{\mathrm{e}}+J_{\mathrm{g}}+K+1}\left(2 J_{\mathrm{e}}+1\right)\right.\right. \\
&\left.\left.\times \sqrt{\left(2 k_{1}+1\right)\left(2 k_{2}+1\right)}\left\{\begin{array}{ccc}
J_{\mathrm{e}} & J_{\mathrm{g}} & 1 \\
J_{\mathrm{e}} & J_{\mathrm{g}} & 1 \\
K & k_{1} & k_{2}
\end{array}\right\}\left\{\begin{array}{lll}
J_{\mathrm{e}} & J_{\mathrm{e}} & K \\
J_{\mathrm{g}} & J_{\mathrm{g}} & 1
\end{array}\right\}\right)\right]+ \text { idem }(1 \rightarrow 2)
\end{aligned}
$$

— des termes de « pompage-déplacement» $\left(k_{1}+k_{2}+K\right.$ impair $)$ :

$$
\begin{aligned}
-\frac{2 i \Gamma_{1}^{\prime} \Delta E_{2}^{\prime}}{2 J_{\mathrm{g}}+1} \frac{1}{i Q \omega_{0}+\gamma} \sum_{\substack{\boldsymbol{k}_{1}, q_{1} \\
\boldsymbol{k}_{2}, q_{2}}}\left\langle k_{1} k_{2} q_{1} q_{2} \mid K Q\right\rangle \frac{\varphi_{q_{1}}^{\left(k_{1}\right)}\left(\mathbf{e}_{\lambda_{1}}\right) \varphi_{q_{2}}^{\left(k_{2}\right)}\left(\mathbf{e}_{\lambda_{2}}\right)}{i q_{1} \omega_{0}+\gamma} \times \\
\times\left[\mathfrak{B}_{k_{1}}\left(J_{\mathrm{g}}, J_{\mathrm{e}}\right) \frac{\left\langle J_{\mathbf{g}}\left\|V^{(K)}\right\| J_{\mathrm{g}}\right\rangle}{\sqrt{2 K+1}}(-1)^{k_{2}+1} B_{k_{2}}\left(J_{\mathrm{g}}, J_{\mathrm{e}}\right)\right]+\text { idem }(1 \rightarrow 2) .
\end{aligned}
$$

Dans ces expressions, le coefficient entre crochets est un facteur numérique fonction de $k_{1}, k_{2}$ et $K$. Nous le noterons :

$\mathcal{B}_{k_{1}}\left(J_{\mathrm{g}}, J_{\mathrm{e}}\right) C_{\mathrm{p}}\left(k_{1}, k_{2}, K\right)$ pour les termes de pompage-pompage et $\mathcal{B}_{k_{1}}\left(J_{\mathrm{g}}, J_{\mathrm{e}}\right) C_{\mathrm{d}}\left(k_{1}, k_{2}, K\right)$ pour les termes de pompage-déplacement.

Les expressions (26) et (27) peuvent donc s'écrire :

$$
\begin{aligned}
& \frac{\Gamma_{1}^{\prime} \Gamma_{2}^{\prime}}{2 J_{\mathrm{g}}+1} \sum_{k_{1} k_{2}} \mathcal{B}_{k_{1}}\left(J_{\mathrm{g}}, J_{\mathrm{e}}\right) C_{\mathrm{p}}\left(k_{1}, k_{2}, K\right) \beta_{Q}^{(K)} \\
& \frac{2 \Gamma_{1}^{\prime} \Delta E_{2}^{\prime}}{2 J_{\mathrm{g}}+1} \sum_{k_{1} k_{2}} \mathcal{B}_{k_{1}}\left(J_{\mathrm{g}}, J_{\mathrm{e}}\right) C_{\mathrm{d}}\left(k_{1}, k_{2}, K\right) \beta_{Q}^{(K)}
\end{aligned}
$$


avec

$$
\begin{gathered}
\beta_{Q}^{(K)}=c\left(k_{1}+k_{2}+K\right) \sum_{q_{1}, q_{2}}\left\langle k_{1} k_{2} q_{1} q_{2} \mid K Q\right\rangle \frac{\varphi_{q_{1}}^{\left(k_{1}\right)}\left(\mathbf{e}_{\lambda_{1}}\right) \varphi_{q_{2}}^{\left(k_{2}\right)}\left(\mathbf{e}_{\lambda_{2}}\right)}{\left(i Q \omega_{0}+\gamma\right)\left(i q_{1} \omega_{0}+\gamma\right)} \\
c\left(k_{1}+k_{2}+K\right)=\mid \begin{array}{l}
1 \text { si } k_{1}+k_{2}+K \text { est pair } \\
-i \text { si } k_{1}+k_{2}+K \text { est impair } .
\end{array}
\end{gathered}
$$

Dans les coefficients $\beta_{Q}^{(K)}$ se trouve rassemblée toute la dépendance angulaire d'un effet de pompage croisé donné (c'est-à-dire associé à des valeurs de $k_{1}, k_{2}, K$ et $Q$ données).

2.3.4 Dépendance angulaire des effets de pompage croisé. - Le signal détecté est proportionnel à une quantité que nous noterons $s$, combinaison linéaire de différents $\sigma_{Q}^{(K)}$, qui, dans le cas d'un faisceau sonde se propageant parallèlement à $\mathrm{O} z$, se réduit à des expressions simples :

$$
s=\sigma_{0}^{(0)}
$$

pour le signal d'absorption indépendant de la polarisation (en supposant nul l'alignement longitudinal);

$$
s=\sigma_{0}^{(1)}
$$

pour le signal de dichroïsme circulaire;

$$
s=\sigma_{2}^{(2)}+\sigma_{-2}^{(2)}
$$

pour le signal de dichroïsme linéaire selon les axes $\mathrm{Ox}$ et $\mathrm{O} y$;

$$
s=\frac{\sigma_{2}^{(2)}-\sigma_{-2}^{(2)}}{i}
$$

pour le signal de dichroïsme linéaire selon les bissectrices de $\mathrm{O} x$ et $\mathrm{O} y$.

Il sera commode de poser de façon analogue $\zeta=\beta_{0}^{(0)}, \beta_{0}^{(1)}, \beta_{2}^{(2)}+\beta_{-2}^{(2)}$ ou $\left(\beta_{2}^{(2)}-\beta_{-2}^{(2)}\right) / i$, selon la nature du signal de détection.

Supposons que chaque faisceau de pompage prenne alternativement deux états de polarisation $\mathbf{e}_{\lambda}$ et $\mathbf{e}_{\mu}$ (en pratique nous nous limiterons à deux situations : polarisation alternativement $\sigma^{+}$et $\sigma^{-}$où seuls les $\varphi_{q}^{(1)}$ varient et polarisation alternativement linéaire dans le plan $z \mathrm{O} x(\leftrightarrow)$ et linéaire parallèle à $\mathrm{O} y(\uparrow)$ où seuls les $\varphi_{q}^{(2)}$ varient). Calculons alors la quantité

$F(s)=s\left(\mathbf{e}_{\lambda_{1}}, \mathbf{e}_{\lambda_{2}}\right)+s\left(\mathbf{e}_{\mu_{1}}, \mathbf{e}_{\mu_{2}}\right)-s\left(\mathbf{e}_{\lambda_{1}}, \mathbf{e}_{\mu_{2}}\right)-s\left(\mathbf{e}_{\mu_{1}}, \mathbf{e}_{\lambda_{2}}\right)$

qui est, à un facteur près, la partie de $s$ qui change de signe à la fois quand on change $\mathbf{e}_{\lambda_{1}}$ en $\mathbf{e}_{\mu_{1}}$, et $\mathbf{e}_{\lambda_{2}}$ en $\mathbf{e}_{\mu_{2}}$ (partie « doublement impaire »). Il est intéressant de considérer cette quantité car c'est à elle qu'on a accès expérimentalement quand on module simultanément les polarisations des deux faisceaux de pompage (aux fréquences respectives $\omega_{1}$ et $\omega_{2}$ ) et qu'on sélectionne dans le signal détecté la partie modulée à la fois à $\omega_{1}$ et $\omega_{2}$. Celle-ci n'est sensible qu'aux termes croisés présents dans ${ }^{(2)} \sigma_{Q}^{(K)}$ associés à des valeurs déterminées de $k_{1}$ et $k_{2}$. A un facteur numérique près, qui n'est

LE JOURNAL DE PHYSIQUE - T. 43, No 4, AVRIL 1982 fonction que de $k_{1}, k_{2}$ et $K$, la partie doublement modulée du signal détecté est alors égale à :

$$
F(\zeta)=\zeta\left(\mathbf{e}_{\lambda_{1}}, \mathbf{e}_{\lambda_{2}}\right)+\zeta\left(\mathbf{e}_{\mu_{1}}, \mathbf{e}_{\mu_{2}}\right)-\zeta\left(\mathbf{e}_{\lambda_{1}}, \mathbf{e}_{\mu_{2}}\right)-\zeta\left(\mathbf{e}_{\mu_{1}}, \mathbf{e}_{\lambda_{2}}\right)
$$

Les tableaux II et III donnent les valeurs de la fonction $F(\zeta)$ pour différents choix des polarisations $\mathbf{e}_{\lambda}$ et $\mathbf{e}_{\mu}$ de chacun des faisceaux de pompage et des quantités $\zeta$ associées à la détection. Dans le tableau II sont rassemblés les effets de " pompage-pompage » et dans le tableau III les effets de "pompage-déplacement ». Dans les deux cas, on s'est limité à l'interaction du système atomique avec le faisceau 1 puis avec le faisceau 2 : il convient donc partout d'ajouter l'effet symétrique où le système atomique interagit d'abord avec le faisceau 2 puis avec le faisceau 1 (cf. équations (26) et (27)).

On peut commenter de la façon suivante deux effets prévus dans le tableau III :

- Le terme de la première ligne décrit l'effet simple présenté dans la partie 1.3. Chaque faisceau de pompage, polarisé alternativement $\sigma^{+}$et $\sigma^{-}$, produit, par un effet de pompage, une orientation qui est modifiée par l'effet de déplacement dû à l'autre faisceau : cet effet de déplacement, dans le cas d'une polarisation circulaire, peut être décrit comme celui d'un champ magnétique fictif $\mathbf{B}_{\mathrm{f}}$ [13]. Il en résulte une orientation longitudinale selon $\mathrm{O} z$ simultanément modulée aux deux fréquences de modulation des polarisations de pompage.

Cet effet croisé est a priori la somme de trois termes contenant les commutateurs $\left[T_{-1}^{(1)}, T_{1}^{(1)}\right],\left[T_{1}^{(1)}, T_{-1}^{(1)}\right]$ et $\left[T_{0}^{(1)}, T_{0}^{(1)}\right]$. Le dernier étant nul, seules les composantes transversales des orientations créées par chacun des faisceaux apportent une contribution. C'est pourquoi la dépendance en angles de l'effet est en $\sin \theta_{1} \sin \theta_{2}$, et la dépendance en champ magnétique proportionnelle à :

$$
i\left[\frac{1}{\gamma+i \omega_{0}}-\frac{1}{\gamma-i \omega_{0}}\right]=\frac{2 \omega_{0}}{\gamma^{2}+\omega_{0}^{2}}
$$

On retrouve donc les résultats obtenus géométriquement au $\S 1.3$.

On peut remarquer que cet effet croisé est une fonction impaire de $B_{0}$. Ceci était prévisible par des 
Tableau II. - Termes croisés en $\Gamma_{1}^{\prime} \Gamma_{2}^{\prime}$ (pompage par 1 puis par 2)

[Crossed $\Gamma_{1}^{\prime} \Gamma_{2}^{\prime}$ terms (pumping by 1 then by 2).]

\begin{tabular}{|c|c|c|c|}
\hline $\begin{array}{r}\text { Polari } \\
\text { des fa } \\
\mathbf{e}_{\lambda_{1}}, \mathbf{e}_{\mu_{1}}\end{array}$ & $\begin{array}{l}\text { ations } \\
\text { sceaux } \\
\mathbf{e}_{\lambda_{2}}, \mathbf{e}_{\mu_{2}}\end{array}$ & $\begin{array}{c}\text { Quantité } \zeta \\
\text { sélectionnée } \\
\text { par la } \\
\text { détection }\end{array}$ & Valeur prise par la fonction $F(\zeta)$ correspondante \\
\hline$\sigma^{+}, \sigma^{-}$ & $\sigma^{+}, \sigma^{-}$ & $\begin{array}{c}\beta_{0}^{(0)} \\
\beta_{2}^{(2)}+\beta_{-2}^{(2)} \\
\frac{\beta_{2}^{(2)}-\beta_{-2}^{(2)}}{i}\end{array}$ & $\begin{array}{l}-\frac{2}{\sqrt{3}}\left[\frac{\cos \theta_{1} \cos \theta_{2}}{\gamma^{2}}+\frac{\sin \theta_{1} \sin \theta_{2}}{\gamma^{2}+\omega_{0}^{2}}\right] \\
2 \sin \theta_{1} \sin \theta_{2} \frac{\gamma^{2}-2 \omega_{0}^{2}}{\left(\gamma^{2}+\omega_{0}^{2}\right)\left(\gamma^{2}+4 \omega_{0}^{2}\right)} \\
2 \sin \theta_{1} \sin \theta_{2} \frac{-3 \gamma \omega_{0}}{\left(\gamma^{2}+\omega_{0}^{2}\right)\left(\gamma^{2}+4 \omega_{0}^{2}\right)}\end{array}$ \\
\hline$\leftrightarrow, \uparrow$ & $\sigma^{+}, \sigma^{-}$ & $\beta_{0}^{(1)}$ & $\frac{\sqrt{6}}{\sqrt{5}}\left[\frac{\sin ^{2} \theta_{1} \cos \theta_{2}}{\gamma^{2}}-\frac{\sin \theta_{1} \cos \theta_{1} \sin \theta_{2}}{\gamma^{2}+\omega_{0}^{2}}\right]$ \\
\hline 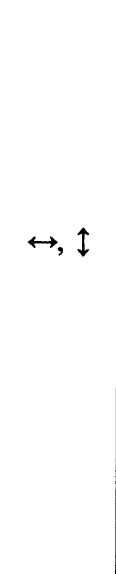 & $\leftrightarrow, \uparrow$ & $\begin{array}{l}\beta_{2}^{(2)}+\beta_{-2}^{(2)} \\
\frac{\beta_{2}^{(2)}-\beta_{-2}^{(2)}}{i}\end{array}$ & $\begin{array}{l}\frac{1}{2 \sqrt{5}}\left[\frac{3 \sin ^{2} \theta_{1} \sin ^{2} \theta_{2}}{\gamma^{2}}+\frac{4 \sin \theta_{1} \cos \theta_{1} \sin \theta_{2} \cos \theta_{2}}{\gamma^{2}+\omega_{0}^{2}}+\right. \\
\left.+\frac{\left(\cos ^{2} \theta_{1}+1\right)\left(\cos ^{2} \theta_{2}+1\right)}{\gamma^{2}+4 \omega_{0}^{2}}\right] \\
\frac{\sqrt{3}}{\sqrt{7}}\left[\frac{\sin ^{2} \theta_{1}\left(\cos ^{2} \theta_{2}+1\right)}{\gamma^{2}+4 \omega_{0}^{2}}-\frac{2 \sin \theta_{1} \cos \theta_{1} \sin \theta_{2} \cos \theta_{2}\left(\gamma^{2}-2 \omega_{0}^{2}\right)}{\left(\gamma^{2}+\omega_{0}^{2}\right)\left(\gamma^{2}+4 \omega_{0}^{2}\right)}+\right. \\
\left.+\frac{\sin ^{2} \theta_{2}\left(\cos ^{2} \theta_{1}+1\right)\left(\gamma^{2}-4 \omega_{0}^{2}\right)}{\left(\gamma^{2}+4 \omega_{0}^{2}\right)^{2}}\right] \\
-\frac{\sqrt{3}}{\sqrt{7}}\left[\frac{\sin ^{2} \theta_{1}\left(\cos ^{2} \theta_{2}+1\right) \times 2 \omega_{0}}{\gamma\left(\gamma^{2}+4 \omega_{0}^{2}\right)}-\frac{\sin \theta_{1} \cos \theta_{1} \sin \theta_{2} \cos \theta_{2} \times 6 \gamma \omega_{0}}{\left(\gamma^{2}+\omega_{0}^{2}\right)\left(\gamma^{2}+4 \omega_{0}^{2}\right)}+\right. \\
\left.+\frac{\sin ^{2} \theta_{2}\left(\cos ^{2} \theta_{1}+1\right) \times 4 \gamma \omega_{0}}{\left(\gamma^{2}+4 \omega_{0}^{2}\right)^{2}}\right]\end{array}$ \\
\hline
\end{tabular}

Tableau III. - Termes croisés en $\Gamma_{1}^{\prime} \Delta E_{2}^{\prime}$ (pompage par 1 puis déplacement par 2).

[Crossed $\Gamma_{1}^{\prime} \Delta E_{2}^{\prime}$ terms (pumping by 1 then shift by 2).]

\begin{tabular}{|c|c|c|c|}
\hline $\begin{array}{r}\text { Polari } \\
\text { des fa } \\
\mathbf{e}_{\lambda_{1}}, \mathbf{e}_{\mu_{1}}\end{array}$ & $\begin{array}{l}\text { ations } \\
\text { sceaux } \\
\mathbf{e}_{\lambda_{2}}, \mathbf{e}_{\mu_{2}}\end{array}$ & $\begin{array}{l}\text { Quantité } \zeta \\
\text { sélectionnée } \\
\text { par la } \\
\text { détection }\end{array}$ & Valeur prise par la fonction $F(\zeta)$ correspondante \\
\hline$\sigma^{+}, \sigma^{-}$ & $\sigma^{+}, \sigma^{-}$ & $\beta_{0}^{(1)}$ & $\frac{\sqrt{2}}{\gamma} \sin \theta_{1} \sin \theta_{2} \frac{\omega_{0}}{\gamma^{2}+\omega_{0}^{2}}$ \\
\hline$\leftrightarrow, \uparrow$ & $\sigma^{+}, \sigma^{-}$ & $\begin{array}{l}\beta_{2}^{(2)}+\beta_{-2}^{(2)} \\
\frac{\beta_{2}^{(2)}-\beta_{-2}^{(2)}}{i}\end{array}$ & $\begin{array}{l}\frac{2}{\sqrt{3}}\left[\sin \theta_{1} \cos \theta_{1} \sin \theta_{2} \frac{3 \gamma \omega_{0}}{\left(\gamma^{2}+\omega_{0}^{2}\right)\left(\gamma^{2}+4 \omega_{0}^{2}\right)}\right. \\
\left.\quad+\cos \theta_{2}\left(\cos ^{2} \theta_{1}+1\right) \frac{4 \gamma \omega_{0}}{\left(\gamma^{2}+4 \omega_{0}^{2}\right)^{2}}\right] \\
\frac{2}{\sqrt{3}}\left[\sin \theta_{1} \cos \theta_{1} \sin \theta_{2} \frac{\gamma^{2}-2 \omega_{0}^{2}}{\left(\gamma^{2}+\omega_{0}^{2}\right)\left(\gamma^{2}+4 \omega_{0}^{2}\right)}+\right. \\
\left.+\cos \theta_{2}\left(\cos ^{2} \theta_{1}+1\right) \frac{\gamma^{2}-4 \omega_{0}^{2}}{\left(\gamma^{2}+4 \omega_{0}^{2}\right)^{2}}\right]\end{array}$ \\
\hline$\leftrightarrow, \uparrow$ & $\leftrightarrow, \uparrow$ & $\beta_{0}^{(1)}$ & $\begin{aligned}-\frac{\sqrt{2}}{\gamma \sqrt{5}}\left[\sin \theta_{1} \cos \theta_{1} \sin \theta_{2}\right. & \cos \theta_{2} \frac{\omega_{0}}{\gamma^{2}+\omega_{0}^{2}}+ \\
& \left.+\left(\cos ^{2} \theta_{1}+1\right)\left(\cos ^{2} \theta_{2}+1\right) \frac{\omega_{0}}{\gamma^{2}+4 \omega_{0}^{2}}\right]\end{aligned}$ \\
\hline
\end{tabular}


considérations de symétrie : en effet, dans une symétrie par rapport au plan $z \mathrm{O} x$ des trois faisceaux, les polarisations circulaires des deux faisceaux de pompage sont changées de signe, et le terme source de l'effet croisé est inchangé; par contre, dans cette symétrie, l'orientation résultante $\left\langle J_{z}\right\rangle$ change de signẹ ainsi que le champ magnétique $B_{0}$.

- Le terme de la dernière ligne est également un effet d'orientation longitudinale, résultant cette fois-ci du couplage entre les alignements créés par chacun des deux faisceaux. Un tel effet, prévu théoriquement par Lombardi [23], a déjà été mis en évidence dans des situations expérimentales où la vitesse des atomes n'est pas sélectionnée [23, 24].

Dans le cas de polarisations linéaires, soit perpendiculaires au plan $z \mathrm{Ox}$, soit contenues dans ce plan, le plan des faisceaux est, comme pour l'effet croisé précédent, un plan de symétrie pour l'excitation. L'orientation résultante $\left\langle J_{z}\right\rangle$ est donc également une fonction impaire de $B_{0}$.

Remarque. - Les termes associés à $\beta_{0}^{(0)}$ dans le tableau II décrivent le «trou de population 》 créé par un effet de pompage croisé entre les deux faisceaux polarisés tous deux circulairement ou tous deux linéairement. En facteur de ces termes, se trouve le coefficient $C_{\mathrm{p}}(k, k, 0)$. Le calcul montre que ce dernier a la forme simple suivante :

$$
\begin{aligned}
C_{\mathrm{p}}(k, k, 0)=(-1)^{J_{\mathrm{e}}+J_{\mathrm{g}}+k} & \sqrt{\frac{2 k+1}{2 J_{\mathrm{g}}+1}} \times \\
& \times\left\{\begin{array}{ccc}
1 & 1 & k \\
J_{\mathrm{g}} & J_{\mathrm{g}} & J_{\mathrm{e}}
\end{array}\right\}\left(1-\frac{\Gamma_{\mathrm{r}}}{\Gamma}\right) .
\end{aligned}
$$

Dans le cas où $\Gamma_{\mathrm{r}}=\Gamma$, c'est-à-dire où les deux niveaux $\mathrm{e}$ et $\mathrm{g}$ forment un système fermé, cette expression est nulle : il ne peut y avoir d'effet de pompage croisé sur la population du niveau $\mathrm{g}$ (il s'agit ici de la somme des populations des sous-niveaux Zeeman du niveau g, qu'on a supposé sans structure hyperfine).

2.4 CAlCUl DU SIGNAL DÉTECTÉ. - 2.4.1 Généralités. - Nous avons calculé dans les paragraphes précédents les termes de pompage croisés à l'ordre le plus bas dans la matrice densité $\sigma_{\mathrm{g}}$ d'un atome de vitesse $\mathbf{v}$ donnée. La valeur moyenne d'une observable $\mathcal{A}$ dans ce niveau est donc une fonction de $\mathbf{v}$ qui s'écrit :

$$
\langle\mathcal{A}\rangle(\mathbf{v})=\operatorname{Tr}\left\{\sigma_{\mathrm{g}}(\mathbf{v}) \cdot \mathcal{A}\right\} .
$$

Elle est détectée par un faisceau sonde de polarisation donnée, de fréquence angulaire $\Omega_{\mathrm{L}}$ (les deux faisceaux pompe et le faisceau sonde sont issus du même laser monomode), et se propageant dans la direction $\mathrm{O} z$, dont on mesure l'absorption par le gaz atomique étudié. L'intensité du faisceau sonde est supposée suffisamment faible pour qu'on puisse négliger tout phénomène de pompage ou de relaxation associé à ce faisceau. D'autre part, dans notre cas où le niveau $\mathrm{g}$ a une durée de vie $1 / \gamma$ beaucoup plus longue que celle de la cohérence optique $\tau_{\text {eg }}$, on peut montrer (lorsque la largeur Doppler est beaucoup plus grande que la largeur naturelle de la transition) que les termes résultant de l'interaction cohérente de l'atome avec les faisceaux de pompage et le faisceau sonde sont négligeables après intégration sur les vitesses des atomes (cf. appendice 1). Le signal s'obtient donc en calculant la réponse au faisceau sonde du système atomique préalablement préparé par les deux faisceaux de pompage.

Les observables détectées par le faisceau sonde dépendent de la polarisation de celui-ci. En modulant de façon appropriée cette polarisation, on peut sélectionner une observable déterminée $\mathcal{A}$. Dans le cas d'un signal d'absorption $\left({ }^{4}\right)$, la contribution des atomes de vitesse $\mathbf{v}$ s'écrit [6] :

$$
S_{\mathrm{v}}\left(\Omega_{\mathrm{L}}\right) \propto \frac{\Gamma_{\mathrm{eg}}}{\left(\Omega_{\mathrm{L}}-\Omega_{0}-\mathbf{k}_{\mathrm{d}} \cdot \mathrm{v}\right)^{2}+\Gamma_{\mathrm{eg}}^{2}}\langle\mathcal{A}\rangle(\mathbf{v})
$$

$\mathbf{k}_{\mathrm{d}}$ est le vecteur d'onde du faisceau de détection :

$$
\mathbf{k}_{\mathrm{d}} \cdot \mathbf{v}=k v_{z}
$$

$k=\Omega_{\mathrm{L}} / c$ est le module du vecteur d'onde du faisceau laser.

La dépendance en vitesse de $\langle\mathcal{A}\rangle$ (v) provient de celle de $\sigma_{\mathrm{g}}(\mathbf{v})$. On s'intéresse ici uniquement au signal résultant des effets de pompage croisés à l'ordre le plus bas en intensité. Le calcul du signal fait donc intervenir les expressions (26) et (27) qui donnent les termes croisés dans ${ }^{(2)} \sigma_{\mathrm{g} Q}^{(K)}$. Dans ces expressions la dépendance en vitesse provient uniquement des produits $\Gamma_{1}^{\prime} \Gamma_{2}^{\prime}$ et $\Gamma_{1}^{\prime} \Delta E_{2}^{\prime}$ où :

$$
\Gamma_{1,2}^{\prime} \propto \frac{\Gamma_{\mathrm{eg}}}{\Gamma_{\mathrm{eg}}^{2}+\left(\Omega_{\mathrm{L}}-\Omega_{0}-\mathbf{k}_{1,2} \cdot \mathrm{v}\right)^{2}}
$$

et

$$
\Delta E_{1,2}^{\prime} \propto \frac{\Omega_{\mathrm{L}}-\Omega_{0}-\mathbf{k}_{1,2} \cdot \mathbf{v}}{\Gamma_{\mathrm{eg}}^{2}+\left(\Omega_{\mathrm{L}}-\Omega_{0}-\mathbf{k}_{1,2} \cdot \mathbf{v}\right)^{2}}
$$

$\mathbf{k}_{1}$ et $\mathbf{k}_{2}$ sont les vecteurs d'onde respectifs des deux faisceaux de pompage; si $\theta_{1}$ et $\theta_{2}$ sont les angles algébriques que font, dans le plan $z \mathrm{Ox}$ orienté, ces deux vecteurs avec $\mathrm{O} z$, on a :

$$
\mathbf{k}_{1,2} \cdot \mathbf{v}=k\left(v_{z} \cos \theta_{1,2}+v_{x} \sin \theta_{1,2}\right) .
$$

Le signal d'absorption associé aux atomes de vitesse $\mathbf{v}$ donnée dépend donc des composantes $v_{x}$ et $v_{z}$ de cette

(4) Pour simplifier, nous nous limiterons dans cet article à l'étude des signaux d'absorption. Cependant, si l'on dispose un analyseur de polarisation sur le faisceau de détection après la traversée du gaz atomique, il est possible de mesurer la dispersion optique de ce gaz; le calcul des signaux eorrespondants serait très semblable à celui que nous présentons. 
vitesse par l'intermédiaire de trois facteurs : une fonction d'absorption de Lorentz liée à la détection; deux fonctions d'absorption de Lorentz liées au pompage, s'il s'agit de termes croisés "pompagepompage", ou l'une d'absorption et l'autre de dispersion. s'il s'agit de termes croisés "pompagedéplacement $"$.

2.4.2 Intégration sur les vitesses. - Pour calculer le signal total détecté pour une fréquence donnée $\Omega_{\mathrm{L}}$, il faut sommer les signaux correspondant à toutes les classes de vitesse présentes dans la cellule. Les fonctions à intégrer étant beaucoup plus étroites que la largeur Doppler résultant de la vitesse thermique des atomes, nous remplacerons dans l'intégrale la répartition des vitesses correspondante par une constante (approximation de largeur Doppler infinie). Le signal total est donc proportionnel $\left(^{5}\right)$ à l'intégrale sur les composantes $v_{x}$ et $v_{z}$ de la vitesse (la composante $v_{y}$ ne joue aucun rôle), du produit des trois fonctions présentées plus haut. Nous allons calculer cette intégrale, d'abord dans le cas d'un signal de " pompagepompage ", puis dans le cas d'un signal de « pompagedéplacement $»$.

- Signal de " pompage-pompage ». - L'intégrale s'écrit :

$$
\begin{aligned}
\mathfrak{J}=\int_{-\infty}^{+\infty} & \int_{-\infty}^{+\infty} \mathrm{d} v_{x} \mathrm{~d} v_{z} \Gamma_{\mathrm{eg}}^{3} \frac{1}{\Gamma_{\mathrm{eg}}^{2}+\left(\Omega_{\mathrm{L}}-\Omega_{0}-k v_{z}\right)^{2}} \times \\
& \times \frac{1}{\Gamma_{\mathrm{eg}}^{2}+\left[\Omega_{\mathrm{L}}-\Omega_{0}-k\left(v_{z} \cos \theta_{1}+v_{x} \sin \theta_{1}\right)\right]^{2}} \\
& \times \frac{1}{\Gamma_{\mathrm{eg}}^{2}+\left[\Omega_{\mathrm{L}}-\Omega_{0}-k\left(v_{z} \cos \theta_{2}+v_{x} \sin \theta_{2}\right)\right]^{2}} .
\end{aligned}
$$

L'intégration sur $v_{x}$, qui ne fait intervenir que les deux fonctions d'absorption de Lorentz liées au pompage, n'est rien d'autre que le produit de convolution de ces deux fonctions. Le résultat de ce produit de convolution, qui se calcule simplement par transformée de Fourier, redonne une fonction d'absorption de Lorentz $\left({ }^{6}\right)$. L'intégration sur $v_{z}$, produit de convolution de celle-ci avec la fonction d'absorption de Lorentz liée à la détection, se fait de la même manière. On obtient finalement :

$$
J=C \frac{1}{X^{2}+L^{2}}
$$

$\left({ }^{5}\right)$ Pour un effet de pompage croisé déterminé $\left(k_{1}, k_{2}, K\right.$ fixés), le coefficient de proportionnalité est le produit de la fonction $F(\zeta)$ portée dans le tableau II (resp. III) par le terme entre crochets dans l'équation (26) (resp. équation (27)).

$$
\text { ( } \begin{aligned}
\int_{-\infty}^{+\infty} \frac{1}{x^{2}+a^{2}} \frac{1}{(y-x)^{2}+b^{2}} \mathrm{~d} x & = \\
& =\frac{\pi \frac{|a|+|b|}{|a b|}}{y^{2}+(|a|+|b|)^{2}} .
\end{aligned}
$$

Les paramètres $X, L$ et $C$ peuvent être déduits du calcul détaillé de l'intégrale, où l'on est amené à faire plusieurs changements de variables; ils valent :

$$
\begin{aligned}
& C=\frac{\pi^{2} \Gamma_{\mathrm{eg}}}{k^{4}} \frac{\left|\sin \theta_{1}\right|+\left|\sin \theta_{2}\right|+\left|\sin \left(\theta_{1}-\theta_{2}\right)\right|}{\sin ^{2}\left(\theta_{1}-\theta_{2}\right)} \\
& X=\frac{\Omega_{\mathrm{L}}-\Omega_{0}}{k}\left(1-\frac{\sin \theta_{1}-\sin \theta_{2}}{\sin \left(\theta_{1}-\theta_{2}\right)}\right) \\
& L=\frac{\Gamma_{\mathrm{eg}}}{k}\left(1+\frac{\left|\sin \theta_{1}\right|+\left|\sin \theta_{2}\right|}{\left|\sin \left(\theta_{1}-\theta_{2}\right)\right|}\right) .
\end{aligned}
$$

Dans ces expressions, les valeurs absolues viennent de ce que, dans les produits de convolution de fonctions de Lorentz, les largeurs s'ajoutent en valeur absolue.

- Signal de «pompage-déplacement ». - Dans ce cas l'intégrale à calculer s'écrit :

$$
\begin{aligned}
J^{\prime}=\int_{-\infty}^{+\infty} & \int_{-\infty}^{+\infty} \mathrm{d} v_{x} \mathrm{~d} v_{z} \Gamma_{\mathrm{eg}}^{2} \frac{1}{\Gamma_{\mathrm{eg}}^{2}+\left(\Omega_{\mathrm{L}}-\Omega_{0}-k v_{z}\right)^{2}} \times \\
& \times \frac{1}{\Gamma_{\mathrm{eg}}^{2}+\left[\Omega_{\mathrm{L}}-\Omega_{0}-k\left(v_{z} \cos \theta_{1}+v_{x} \sin \theta_{1}\right)\right]^{2}} \\
& \times \frac{\Omega_{\mathrm{L}}-\Omega_{0}-k\left(v_{z} \cos \theta_{2}+v_{x} \sin \theta_{2}\right)}{\Gamma_{\mathrm{eg}}^{2}+\left[\Omega_{\mathrm{L}}-\Omega_{0}-k\left(v_{z} \cos \theta_{2}+v_{x} \sin \theta_{2}\right)\right]^{2}} .
\end{aligned}
$$

De façon analogue à l'intégrale précédente, deux produits de convolution successifs apparaissent dans le calcul de $\mathfrak{J}^{\prime}$; chacun, qui concerne une fonction d'absorption et une fonction de dispersion, redonne après intégration une fonction de dispersion $\left({ }^{7}\right)$. On obtient :

$$
\mathfrak{J}^{\prime}=C^{\prime} \frac{X}{X^{2}+L^{2}}
$$

avec :

$$
C^{\prime}=\frac{\pi^{2}}{k^{3}} \frac{\sin \theta_{1}}{\left|\sin \theta_{1}\right|} \frac{1}{\sin \left(\theta_{2}-\theta_{1}\right)} .
$$

La variable $X$ et la largeur $L$ sont les mêmes que précédemment.

2.4.3 Effet du signal symétrique $(1 \leftrightarrow 2)$. - Les signaux calculés dans le paragraphe précédent sont ceux qui résultent d'un effet de pompage croisé où le faisceau 1 agit d'abord (pompage) et le faisceau 2 ensuite (pompage ou déplacement). On doit leur ajouter les signaux résultant de l'effet croisé symétrique (action du faisceau 2 puis du faisceau 1), et qui intervient toujours simultanément dans une situation

$$
\text { (7) } \begin{aligned}
\int_{-\infty}^{+\infty} \frac{1}{x^{2}+a^{2}} \frac{x-y}{(y-x)^{2}+b^{2}} \mathrm{~d} x & = \\
& =\frac{\pi}{|a|} \frac{y}{y^{2}+(|a|+|b|)^{2}} .
\end{aligned}
$$


expérimentale donnée. Ces signaux se calculent de façon identique aux précédents; il suffit simplement dans les résultats obtenus d'échanger les indices 1 et 2 .

- Dans le cas d'un signal de "pompage-pompage ", les deux faisceaux de pompage jouent dans le calcul précédent exactement le même rôle, et l'intégrale $\mathfrak{J}$ est invariante dans l'échange des indices 1 et 2 . Le coefficient de proportionnalité entre le signal détecté et l'intégrale dépend en général de l'ordre dans lequel l'atome a interagi avec les faisceaux. Cependant, on peut montrer que si $k_{1}=k_{2}$ et $Q=0$ ce coefficient est lui aussi invariant dans l'échange des indices 1 et 2. Pour simplifier la discussion, nous nous limiterons à ce cas particulier où tenir compte de l'effet symétrique revient donc uniquement à doubler le signal déjà calculé.

- Par contre, dans le cas d'un signal de " pompagedéplacement", les deux faisceaux ne jouent pas le même rôle. L'échange des indices 1 et 2 , s'il laisse invariant $X$ et $L$, n'est pas indifférent dans $C^{\prime}$. Deux cas peuvent se présenter :

- ou bien $\sin \theta_{1}$ et $\sin \theta_{2}$ sont de signes opposés ; dans ce cas, la constante $C^{\prime}$ est la même pour les deux effets symétriques, et le signal est simplement doublé comme pour le signal de pompage-pompage;

- ou bien $\sin \theta_{1}$ et $\sin \theta_{2}$ sont de même signe;
$C^{\prime}$ change alors de signe par échange de 1 et 2 et les signaux résultant des deux effets symétriques s'annulent exactement.

En conséquence, dans le cas particulier considéré, les signaux de "pompage-déplacement » ne sont visibles que dans certaines conditions géométriques : si l'on choisit par définition $\theta_{1}$ et $\theta_{2}$ tels que $\left|\theta_{1}\right|$ et $\left|\theta_{2}\right|<\pi$, on doit avoir $\theta_{1}$ et $\theta_{2}$ de signes opposés, c'est-à-dire que les deux faisceaux de pompage (orientés par leurs directions de propagation) doivent être situés dans le plan $z \mathrm{O} x$ de part et d'autre du faisceau sonde.

2.4.4 Etude de la largeur en fréquence des signaux. - Les valeurs de $X$ et $L$ étant identiques, les signaux en absorption ou en dispersion (quand ils existent) ont la même largeur en fréquence. Celle-ci vaut :

$$
\mathcal{L}=2 \Gamma_{\mathrm{eg}} \frac{1+\frac{\left|\sin \theta_{1}\right|+\left|\sin \theta_{2}\right|}{\left|\sin \left(\theta_{1}-\theta_{2}\right)\right|}}{\left|1-\frac{\sin \theta_{1}-\sin \theta_{2}}{\sin \left(\theta_{1}-\theta_{2}\right)}\right|} .
$$

Cette expression se simplifie considérablement si l'on fait des hypothèses concernant les signes respectifs de $\sin \theta_{1}, \sin \theta_{2}$ et $\sin \left(\theta_{1}-\theta_{2}\right)$. Nous utiliserons les quatre relations suivantes :

$$
\begin{aligned}
& \sin \left(\theta_{1}-\theta_{2}\right)-\left(\sin \theta_{1}-\sin \theta_{2}\right)=4 \sin \frac{\theta_{1}}{2} \sin \frac{\theta_{2}}{2}\left(\sin \frac{\theta_{1}}{2} \cos \frac{\theta_{2}}{2}-\sin \frac{\theta_{2}}{2} \cos \frac{\theta_{1}}{2}\right) \\
& \sin \left(\theta_{1}-\theta_{2}\right)+\left(\sin \theta_{1}-\sin \theta_{2}\right)=4 \cos \frac{\theta_{1}}{2} \cos \frac{\theta_{2}}{2}\left(\sin \frac{\theta_{1}}{2} \cos \frac{\theta_{2}}{2}-\sin \frac{\theta_{2}}{2} \cos \frac{\theta_{1}}{2}\right) \\
& \sin \left(\theta_{1}-\theta_{2}\right)+\left(\sin \theta_{1}+\sin \theta_{2}\right)=4 \sin \frac{\theta_{1}}{2} \cos \frac{\theta_{2}}{2}\left(\sin \frac{\theta_{1}}{2} \sin \frac{\theta_{2}}{2}+\cos \frac{\theta_{1}}{2} \cos \frac{\theta_{2}}{2}\right) \\
& \sin \left(\theta_{1}-\theta_{2}\right)-\left(\sin \theta_{1}+\sin \theta_{2}\right)=-4 \sin \frac{\theta_{2}}{2} \cos \frac{\theta_{1}}{2}\left(\sin \frac{\theta_{1}}{2} \sin \frac{\theta_{2}}{2}+\cos \frac{\theta_{1}}{2} \cos \frac{\theta_{2}}{2}\right) .
\end{aligned}
$$

Rappelons qu'on a choisi par hypothèse :

$$
-\pi<\theta_{1}<+\pi \text { et }-\pi<\theta_{2}<+\pi \text {. }
$$

Différents cas peuvent se présenter, qui sont schématisés sur la figure 3 .

- Cas 1. - $\theta_{1}$ et $\theta_{2}$ sont de signes opposés et tels que $\left|\overline{\theta_{1}}-\theta_{2}\right|>\pi$; on a donc

$$
\left\{\begin{array} { l } 
{ \theta _ { 1 } > 0 } \\
{ \theta _ { 2 } < 0 } \\
{ \operatorname { s i n } ( \theta _ { 1 } - \theta _ { 2 } ) < 0 }
\end{array} \text { ou bien } \left\{\begin{array}{l}
\theta_{1}<0 \\
\theta_{2}>0 \\
\sin \left(\theta_{1}-\theta_{2}\right)>0 .
\end{array}\right.\right.
$$

Dans ce cas, la largeur du signal est simplement :

$$
\mathcal{L}_{1}=2 \Gamma_{\mathrm{eg}} \text {. }
$$

Elle est égale à la largeur naturelle de la transition considérée, comme dans le cas d'une expérience de pompage optique sélectif en vitesses à un faisceau.

- Cas 2. - $\theta_{1}$ et $\theta_{2}$ sont de signes opposés, et tels que $\left|\overline{\theta_{1}-} \theta_{2}\right|<\pi ;$ on a donc ${ }^{3}$ :

$\left\{\begin{array}{l}\theta_{1}>0 \\ \theta_{2}<0 \\ \sin \left(\theta_{1}-\theta_{2}\right)>0\end{array}\right.$ ou bien $\left\{\begin{array}{l}\theta_{1}<0 \\ \theta_{2}>0 \\ \sin \left(\theta_{1}-\theta_{2}\right)<0 .\end{array}\right.$

Dans ce cas, la largeur du signal s'écrit, après transformation :

$$
\mathfrak{L}_{2}=2 \Gamma_{\mathrm{eg}}\left|\operatorname{cotg} \frac{\theta_{1}}{2} \operatorname{cotg} \frac{\theta_{2}}{2}\right| .
$$

Cette largeur, qui est toujours plus grande que la largeur naturelle, tend vers celle-ci quand $\left|\theta_{1}-\theta_{2}\right|$ tend vers $\pi$ (faisceaux de pompage se propageant en sens opposés). Si $\theta_{1}$ ou $\theta_{2}$ est nul (un des faisceaux 


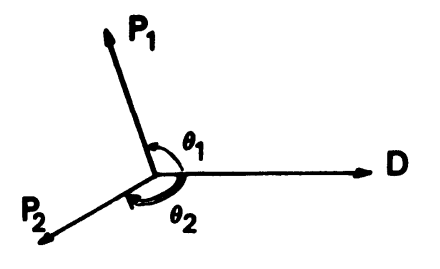

cas 1

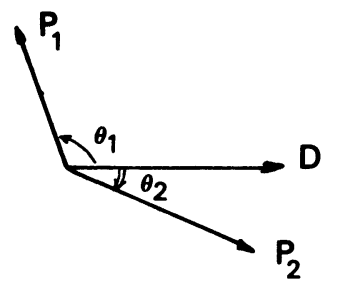

cas 2

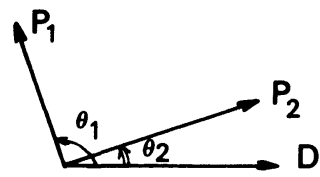

cas 3

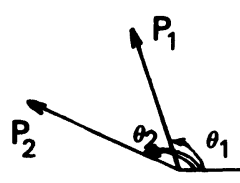

cas. 4

Fig. 3. - Les 4 schémas illustrent les 4 cas possibles de disposition respective des faisceaux de pompage entre eux et par rapport au faisceau sonde $D$; la largeur en fréquence des signaux n'est égale à la largeur naturelle de la transition que dans le cas 1 .

[Four possible ways to set the relative positions of the two pumping beams $P_{1}$ and $P_{2}$ and the detection beam $D$. Only case 1 gives a signal with a natural linewidth (no Doppler broadening).]

de pompage se propage dans le même sens que la sonde), $\mathcal{L}_{2}$ devient infinie (en fait, on aurait obtenu la largeur Doppler si on ne l'avait pas supposée infinie).

- Cas 3. - $\theta_{1}$ et $\theta_{2}$ sont de même signe, et tels que $\left|\theta_{1}\right|{\overline{>\mid \theta_{2}}}_{2} \mid$; on a donc :

$$
\left\{\begin{array} { l } 
{ \theta _ { 1 } > 0 } \\
{ \theta _ { 2 } > 0 } \\
{ \operatorname { s i n } ( \theta _ { 1 } - \theta _ { 2 } ) > 0 }
\end{array} \text { ou bien } \left\{\begin{array}{l}
\theta_{1}<0 \\
\theta_{2}<0 \\
\sin \left(\theta_{1}-\theta_{2}\right)<0 .
\end{array}\right.\right.
$$

Dans ce cas, la largeur du signal devient :

$$
\mathcal{L}_{3}=2 \Gamma_{\mathrm{eg}} \operatorname{cotg} \frac{\theta_{2}}{2} \operatorname{cotg}\left(\frac{\theta_{1}-\theta_{2}}{2}\right) .
$$

- Cas 4. $-\theta_{1}$ et $\theta_{2}$ sont de même signe, et tels que $\left|\theta_{1}\right|{\overline{<\mid \theta_{2}}}_{2} \mid$; on a donc :

$$
\left\{\begin{array} { l } 
{ \theta _ { 1 } > 0 } \\
{ \theta _ { 2 } > 0 } \\
{ \operatorname { s i n } ( \theta _ { 1 } - \theta _ { 2 } ) < 0 }
\end{array} \text { ou bien } \left\{\begin{array}{l}
\theta_{1}<0 \\
\theta_{2}<0 \\
\sin \left(\theta_{1}-\theta_{2}\right)>0 .
\end{array}\right.\right.
$$

La largeur du signal vaut alors :

$$
\mathcal{L}_{4}=2 \Gamma_{\mathrm{eg}} \operatorname{cotg} \frac{\theta_{1}}{2} \operatorname{cotg}\left(\frac{\theta_{2}-\theta_{1}}{2}\right) .
$$

Les cas 3 et 4, qui ne diffèrent que par la numérotation des faisceaux de pompage, sont identiques physiquement. Dans les deux cas, la largeur trouvée est plus grande que la largeur naturelle et devient très grande, c'est-à-dire égale à la largeur Doppler, quand un faisceau est parallèle à la sonde, ou quand les deux faisceaux de pompage sont parallèles entre eux.

En résumé, la situation où le signal est le plus étroit est celle où les deux faisceaux de pompage sont de part et d'autre de la sonde et où, dans l'espace des vitesses, la zone doublement pompée va à la rencontre de la zone détectée lorsque la fréquence du laser varie (situation de la figure 1). La largeur du signal est alors la largeur naturelle de la transition considérée $\left({ }^{8}\right)$. Dans cette situation, les deux types de signaux (" pompage-pompage » et " pompage-déplacement ») peuvent être détectés.

3. Expériences. - 3.1 PrinciPe. - Nous avons réalisé une expérience de pompage optiqué à deux faisceaux, doublement sélectif en vitesses, sur le niveau métastable ${ }^{3} \mathrm{P}_{2}$ du néon. La cellule contenant les atomes de néon est illuminée par deux faisceaux de pompage étendus, issus du même laser monomode : leur intersection détermine dans la cellule une zone doublement pompée d'environ $10 \mathrm{~cm}^{3}$, qui est traversée par un faisceau sonde non étendu issu du même laser. Les polarisations des trois faisceaux sont modulées à des fréquences différentes de façon que la détection puisse sélectionner un effet croisé déterminé (cf. $§ 2.3 .3$ ).

La transition utilisée, de longueur d'onde $6402 \AA$, est celle qui relie le niveau métastable ${ }^{3} \mathrm{P}_{2}$, de la configuration $2 \mathrm{p}^{5} 3 \mathrm{~s}$, au niveau $2 \mathrm{p}_{9}$ (notation de Paschen) de la configuration supérieure $2 \mathrm{p}^{5} 3 \mathrm{p}$. La largeur naturelle en fréquence de cette transition est d'environ $8 \mathrm{MHz}$. Le niveau $2 \mathrm{p}_{9}$, de moment cinétique $J_{\mathrm{e}}=3$, ne peut se désexciter par émission spontanée que vers le ${ }^{3} \mathrm{P}_{2}$. Les deux niveaux forment donc, en principe, un " système fermé ", dans la mesure où l'on néglige les effets collisionnels de transfert à partir du niveau excité [25]. De fait, nous avons observé des signaux de population, dans une expérience de pompage optique sélectif en vitesses à un seul faisceau, sur cette transition, à la même pression que celle utilisée ici (10 mtorr de néon). Ils sont cependant beaucoup moins importants (environ 10 fois moins) que les signaux d'orientation qu'on peut obtenir dans les mêmes conditions de pression et d'intensité de pompage.

$\left({ }^{8}\right)$ La théorie développée ici est limitée au $1^{\text {er }}$ ordre par rapport à l'intensité de chacun des faisceaux. Des effets de saturation et d'élargissement apparaîtraient bien sûr aux ordres supérieurs. 
3.2 DisPositif EXPÉRIMENTAL. - 3.2.1 La cellule. - Les atomes de néon sont contenus dans une cellule en pyrex sphérique de diamètre $11 \mathrm{~cm}$, munie de deux fenêtres planes de diamètre $5 \mathrm{~cm}$ de façon que la qualité optique du faisceau sonde ne soit pas altérée par la traversée de la cellule. Elle est reliée à un banc de pompage par un dispositif qui permet de vider la cellule, de l'étuver, et de la remplir d'un mélange gazeux de composition et de pression connues (mesurée par une jauge de type Baratron). La pression de remplissage utilisée ici était de 10 mtorr de néon naturel $(90 \%$ $\mathrm{de}^{20} \mathrm{Ne}, 10 \% \mathrm{de}^{22} \mathrm{Ne}$ ).

Une décharge faible est entretenue dans la cellule (champ oscillant à $14 \mathrm{MHz}$, appliqué grâce à deux fils extérieurs à la cellule) de façon à peupler les niveaux métastables du néon.

3.2.2 Les champs magnétiques. - La cellule est placée au centre d'un jeu de trois paires de bobines en position de Helmholtz d'axes deux à deux orthogonaux, destinées à compenser le champ magnétique terrestre et à appliquer un champ longitudinal (parallèle à l'axe $\mathrm{O} z$ de la détection) valant au maximum quelques gauss.

Une bonne compensation du champ terrestre peut être obtenue de la façon suivante : on réalise une expérience de pompage optique sélectif en vitesses à un faisceau de façon à obtenir un signal en pure orientation; dans un champ transverse modéré $(\simeq$ quelques dizaines de $\mathrm{mG}$ ), on annule le champ longitudinal en minimisant le signal; le champ longitudinal étant nul, on annule ensuite les autres composantes en maximisant le signal.

Les champs sont étalonnés avec un milligaussmètre; leur mesure se fait directement par la mesure des courants traversant les bobines.

3.2.3 Le laser à colorant. - La source lumineuse utilisée pour le pompage et la détection est un laser à colorant fonctionnant avec un mélange de Rhodamine 590 et de Rhodamine 640. Il est pompé avec la raie $\lambda=5145 \AA$ d'un laser à argon ionisé. Ce laser à colorant est un modèle CR490 de Coherent Radiation modifié au laboratoire de façon à le rendre monomode (remplacement du miroir de sortie de la cavité par un sélecteur de modes "double Michelson » décrit dans [2], [26]). Il est placé dans une boîte étanche qui permet de faire varier la pression de l'air contenu dans la cavité et donc également la fréquence du laser.

Un système d'asservissement impose à la fréquence du laser de suivre celle d'une cavité Fabry-Pérot extérieure. A cet effet, le signal de transmission du FabryPérot est séparé en deux parties : la partie alternative (fréquence $>1 \mathrm{~Hz}$ ) sert, après intégration et amplification, à piloter la cale piézo-électrique qui contrôle la longueur de la cavité laser ; la partie quasi continue, de son côté, est utilisée au pilotage de la pression dans le laser. De cette façon, un balayage linéaire de la pression dans le Fabry-Pérot induit un balayage linéaire de la fréquence du laser à colorant : on réalise ainsi aisément des balayages de plus de $10 \mathrm{GHz}$.
Ce dispositif a également pour effet de réduire le « jitter» du laser à $\pm 5 \mathrm{MHz}$ environ.

Pour cette expérience, la puissance délivrée par le laser à $6402 \AA$ était de l'ordre de $30 \mathrm{~mW}$, ce qui est largement suffisant pour obtenir des effets de saturation du pompage optique avec des faisceaux étendus.

3.2.4 Le montage optique. - Le faisceau lumineux issu du laser est séparé en trois parties : les deux premières, d'intensités comparables, sont utilisées pour le double pompage optique; la troisième, beaucoup moins intense, sert à la détection. La disposition générale des trois faisceaux est schématisée sur la figure 4. Chaque faisceau traverse un cristal électrooptique (noté $\mathrm{C}_{1}, \mathrm{C}_{2}$ ou $\mathrm{C}$ ) qui module sa polarisation en créneaux, soit alternativement $\sigma^{+}$et $\sigma^{-}$, soit alternativement linéaire dans le plan $z \mathrm{O} x$ des faisceaux et linéaire perpendiculairement à ce plan. Les fréquences de modulation seront notées respectivement $\omega_{1}$ et $\omega_{2}$ pour les faisceaux de pompage, et $\omega$ pour le faisceau de détection. Le diamètre de chaque faisceau de pompage est multipiié par 10 par un jeu de deux lentilles.

Des contraintes géométriques liées aux bobines de champ magnétique nous ont amenés à choisir une géométrie où les deux faisceaux de pompage sont, dans la cellule, symétriques par rapport à l'axe $\mathrm{O} z$ commun à la détection et au champ magnétique : les angles $\theta_{1}$ et $\theta_{2}$ valent respectivement $+135^{\circ}$ et $-135^{\circ}$. Rappelons que, dans cette situation, la théorie

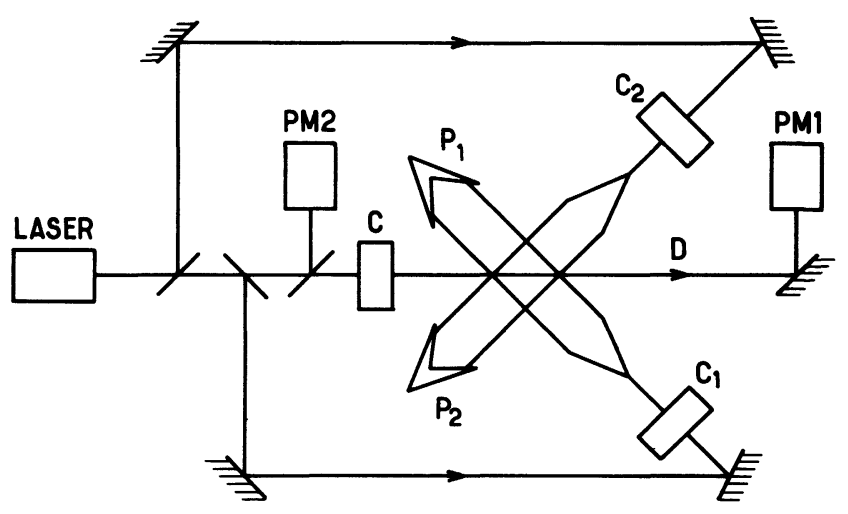

Fig. 4. - Dispositif expérimental : les trois faisceaux $P_{1}, P_{2}$ et $D$ sont coplanaires; $C_{1}, C_{2}$ et $C$ sont les cristaux électrooptiques qui modulent la polarisation de chacun des faisceaux; les photomultiplicateurs PM1 et PM2 délivrent des signaux proportionnels à l'intensité du faisceau sonde respectivement après et avant traversée de la cellule. En mesurant la différence des signaux qu'ils fournissent, on peut s'affranchir presque totalement du bruit d'intensité du laser.

[Scheme of the experiment. The two pumping beams $\mathbf{P}_{1}$ and $P_{2}$ and the detection beam $D$ are coplanar. $C_{1}, C_{2}$ and $C$ are electrooptic polarization modulators. PM1 and PM2 are photomultipliers which deliver signals proportional to the probe beam intensity respectively after and before crossing the cell. Measuring the difference of their signals allows one to reduce the effect of instabilities in the laser intensity, and therefore to improve the signal to noise ratio.] 
prévoit que des signaux de "pompage-déplacement " peuvent être détectés, et que la largeur en fréquence des signaux est minimale (cf. § 2.4).

Une lame séparatrice prélève une partie du faisceau sonde avant sa traversée de la cellule et l'envoie sur un photomultiplicateur (PM2). Les signaux délivrés par les photomultiplicateurs PM1 et PM2 sont donc proportionnels à l'intensité du faisceau sonde avant et après la cellule. Ils sont utilisés pour une méthode de détection différentielle, présentée plus loin, et qui permet d'éliminer presque totalement le bruit dû aux fluctuations d'intensité du laser.

3.2.5 L'électronique de détection. - Elle doit permettre de sélectionner dans le signal d'absorption délivré par le PM1, la composante du signal modulé à la fois aux trois fréquences $\omega, \omega_{1}$ et $\omega_{2}$ de façon à isoler le signal de pompage croisé associé à une observable déterminée. Plutôt que de chercher un signal à une fréquence de la forme $\omega \pm \omega_{1} \pm \omega_{2}$, c'est-à-dire sur l'une des «bandes latérales » de l'excitation et de la détection, il nous a paru préférable, pour ne pas perdre une partie du signal, d'utiliser trois détections synchrones " en cascade " (le signal de sortie de l'une sert de signal d'entrée pour la suivante) démodulant le signal respectivement aux fréquences $\omega, \omega_{1}$ et $\omega_{2}$. Les détections synchrones sont deux PAR 124 et une PAR HR8. Elles démodulent le signal par ordre de fréquences décroissantes, et ces fréquences sont choisies suffisamment différentes pour que toutes les fréquences de modulation du signal d'entrée de chacune restent dans la bande passante de l'amplificateur sélectif correspondant $\left(\omega=18 \mathrm{kHz}, \omega_{1}=1 \mathrm{kHz}\right.$ et $\omega_{2}=80 \mathrm{~Hz}$ ). Une constante de temps minimale est appliquée à la sortie des deux premières, pour qu'elles laissent passer au mieux le signal cherché.

Le signal de sortie de la troisième détection synchrone est envoyé sur la voie $Y$ d'un enregistreur dont la voie $\mathrm{X}$ est alimentée par un signal proportionnel à la pression dans le Fabry-Pérot mentionné plus haut, donc à la fréquence du laser. L'étalonnage de la voie $\mathrm{X}$ est facile à partir du déplacement isotopique ${ }^{20} \mathrm{Ne}-{ }^{22} \mathrm{Ne}$ qui est bien connu.

Pour nous affranchir du bruit d'intensité du laser, nous avons utilisé comme signal d'entrée de la première détection synchrone, non pas le signal délivré par PM1 seul, mais la différence (obtenue par un amplificateur différentiel) des signaux délivrés par PM1 et PM2. Pour que cette méthode soit efficace, il faut que les gains des deux photomultiplicateurs aient été ajustés de façon que ces deux signaux aient des niveaux continus égaux. Comme l'intensité du faisceau sonde, après traversée de la cellule, varie quand on balaie le profil d'absorption de la raie, il a été utile d'asservir la haute tension d'alimentation du PM2 de façon que l'égalité des deux signaux continus soit toujours assurée.

3.3 Résultats. - 3.3.1 Généralités. - Nous avons observé avec ce dispositif expérimental diffé- rents signaux, correspondant soit à un effet de pompage croisé du type "pompage-pompage " (signal variant en absorption avec la fréquence du laser), soit à un effet du type "pompage-déplacement" (signal variant en dispersion avec la fréquence du laser). Le plus grand signal que nous ayons obtenu est de cette deuxième sorte. Il s'agit de l'effet déjà présenté en 1.3 où les polarisations des trois faisceaux sont circulaires, alternativement droites et gauches.

Ce signal a pour amplitude environ $10^{-4}$ de la tension totale délivrée par PM1, le taux maximal d'absorption par le gaz étant de l'ordre de $15 \%$. Cette valeur doit être comparée à celle qu'on obtient dans une expérience de pompage optique sélectif en vitesses avec un seul faisceau, pour une même intensité de pompage et avec une cellule identique; l'amplitude du signal est alors $5 \%$ de la tension totale (taux d'orientation $30 \%$ ). Le facteur 500 perdu en passant d'une expérience à l'autre est dû à plusieurs causes :

- Dans l'expérience à deux faisceaux, on sélectionne deux composantes de la vitesse des atomes au lieu d'une seule : on perd ainsi un facteur 50 à 100 sur le nombre d'atomes participant au signal (en l'absence de «jitter " du laser, ce facteur est théoriquement le rapport de la largeur Doppler à la largeur de la transition).

- On perd également un facteur géométrique dû au fait que le trajet du faisceau sonde dans la région de la cellule où les atomes peuvent être doublement pompés est nettement plus court que dans le cas

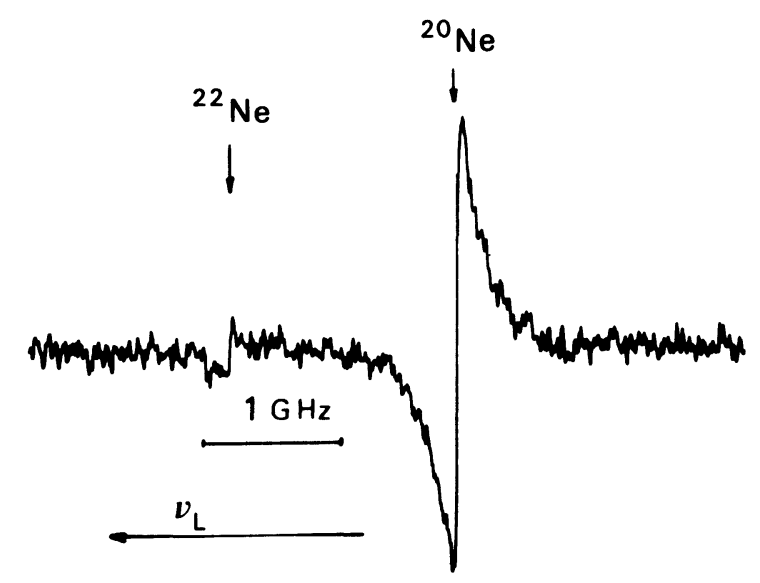

Fig. 5. - Signal «principal» (orientation résultant du couplage de deux orientations) obtenu quand on balaie la fréquence du laser; les deux courbes de dispersion sont dues au ${ }^{20} \mathrm{Ne}$ et au ${ }^{22} \mathrm{Ne}$; l'amplitude du signal correspond à une modification d'environ $10^{-4}$ de l'intensité du faisceau sonde, sa largeur en fréquence (distance entre le minimum et le maximum) est de l'ordre de $25 \mathrm{MHz}$.

[Doubly velocity selective signals obtained as a function of the laser frequency with a mixture of ${ }^{20} \mathrm{Ne}$ and ${ }^{22} \mathrm{Ne}$. The atomic observable detected is the orientation, obtained by the coupling of two orientations. The distance between the maximum and the minimum for each curve is about $25 \mathrm{MHz}$.] 
d'un seul faisceau de pompage où on utilise une géométrie colinéaire.

- Enfin, le deuxième faisceau de pompage ne peut modifier que partiellement les observables crées par le premier.

Le rapport signal sur bruit obtenu pour ce signal que nous appellerons "signal principal " parce qu'il est le plus grand, est de l'ordre de 20.

3.3.2 Etude du signal principal. - La figure 5 montre le signal d'orientation obtenu, par couplage de deux orientations (effet de "pompage-déplacement »), quand on balaie la fréquence du laser. Deux courbes de dispersion sont visibles, d'importance inégales : elles correspondent au même signal pour le ${ }^{20} \mathrm{Ne}$ et le ${ }^{22} \mathrm{Ne}$. Elles sont très nettement séparées, ce qui ne serait pas le cas en présence d'un élargissement Doppler des raies.

Une analyse du profil du signal montre que, bien qu'il s'agisse d'une courbe impaire avec $\delta=\Omega_{\mathrm{L}}-\Omega_{0}$, ce n'est pas une courbe de dispersion de Lorentz : les ailes de la courbe sont trop importantes par rapport aux extrema. Ce fait peut être expliqué par l'existence dans la cellule de collisions qui changent la vitesse des atomes en conservant partiellement leur orientation. On sait en effet que de telles collisions peuvent jouer un rôle dans̄ ces conditions expérimentales [7]. Leur influence sur les signaux de pompage optique croisé est discutée dans l'Appendice 2. On y montre que le profil en fréquence des signaux est élargi mais garde la même parité par rapport à $\Omega_{\mathrm{L}}-\Omega_{0}$, ce qui est en accord avec l'expérience.

On peut définir la largeur du signal comme la distance en fréquence entre le maximum et le minimum de la courbe. Dans des conditions où le signal est désaturé au maximum en atténuant les faisceaux de pompage, nous avons constaté que cette largeur varie avec le champ magnétique longitudinal : elle est environ de $40 \mathrm{MHz}$ en champ faible tandis qu'elle n'est que de $25 \mathrm{MHz}$ dans un champ de $100 \mathrm{mG}$ ou plus. Ce phénomène peut lui aussi être expliqué par la présence de collisions, qui tendent à élargir les courbes expérimentales, mais dont on peut montrer (cf. appendice 2) que l'effet sur la largeur est réduit par un champ magnétique.

Cette dernière valeur est à comparer à celle qu'on obtient dans une expérience de pompage optique sélectif en vitesses avec un seul faisceau et dans des conditions identiques, qui est de $18 \mathrm{MHz}$ environ (rappelons que dans les deux cas la largeur théorique est la largeur naturelle, d'environ $8 \mathrm{MHz}$ ). Dans les deux expériences, l'écart entre la valeur expérimentale et la valeur théorique peut être dû à plusieurs causes :

le « jitter» du laser,

la colinéarité ou la coplanarité imparfaite des faisceaux entre eux,

le parallélisme imparfait de l'un ou de l'autre des faisceaux.

Nous avons étudié également l'amplitude du signal

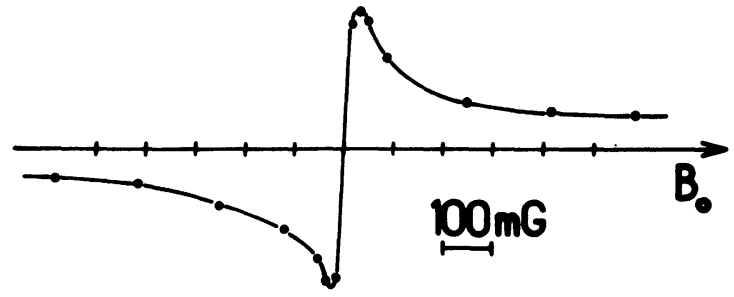

Fig. 6. - Variation de l'amplitude du signal principal avec le champ magnétique longitudinal $B_{0}$; les points sont expérimentaux; la courbe obtenue en reliant les points entre eux a une forme de dispersion, comme prévu théoriquement, mais ses ailes sont plus importantes que celles d'une courbe de Lorentz.

[Amplitude of the signal of figure 5 as a function of the static magnetic field value $B_{0}$. A dispersion shaped curve is obtained, as predicted by theory. The solid line passes through the experimental points and is only a guide to the eye.]

en fonction de celle du champ magnétique longitudinal. La variation correspondante est reportée sur la figure 6. De même que pour le profil en fréquence, la courbe obtenue est impaire avec $B_{0}$, comme prévu théoriquement. Il ne s'agit cependant pas d'une véritable courbe de dispersion de Lorentz : les ailes de la courbe sont trop importantes. Ceci n'est pas explicable par l'effet des collisions $\left({ }^{9}\right)$. Différentes explications peuvent être proposées : taux de relaxation $\gamma$ des atomes métastables dépendant de la vitesse, faible champ magnétique transverse (l'expérience montre en effet qu'un champ magnétique transverse appliqué de quelques $\mathrm{mG}$ suffit pour élargir la courbe considérée de façon notable).

Malgré ces difficultés, une valeur approximative du taux de relaxation moyen des atomes de vitesses nulle dans le plan $z \mathrm{O} x$ peut être déduite de la largeur de la courbe expérimentale obtenue en fonction de $B_{0}$. En désaturant au mieux le signal, nous avons trouvé une demi-largeur de $(25 \pm 5) \mathrm{mG}$, ce qui correspond à $\gamma=(310 \pm 30) \cdot 10^{3} \mathrm{rad} . \mathrm{s}^{-1}$. Cette valeur est en accord avec celle qu'on peut mesurer par une expérience de pompage optique modulé avec un seul faisceau [7].

3.3.3 Autres signaux observés. - Nous avons observé d'autres signaux mais sans pouvoir en mener une étude aussi détaillée, en raison du rapport signal sur bruit obtenu qui est nettement plus petit que celui du signal principal.

- La figure $7 a$ montre le signal d'orientation prévu par Lombardi [23] (cf. 2.3.4), et qui résulte du couplage de deux alignements par un effet de " pompagedéplacement $\%$. Nous avons utilisé des polarisations

$\left({ }^{9}\right)$ Comme montré dans l'appendice 2, les collisions jouent un rôle plus important en champ faible, et devraient donc nous amener à surestimer le signal en champ faible, sans modifier le signal en champ fort. 

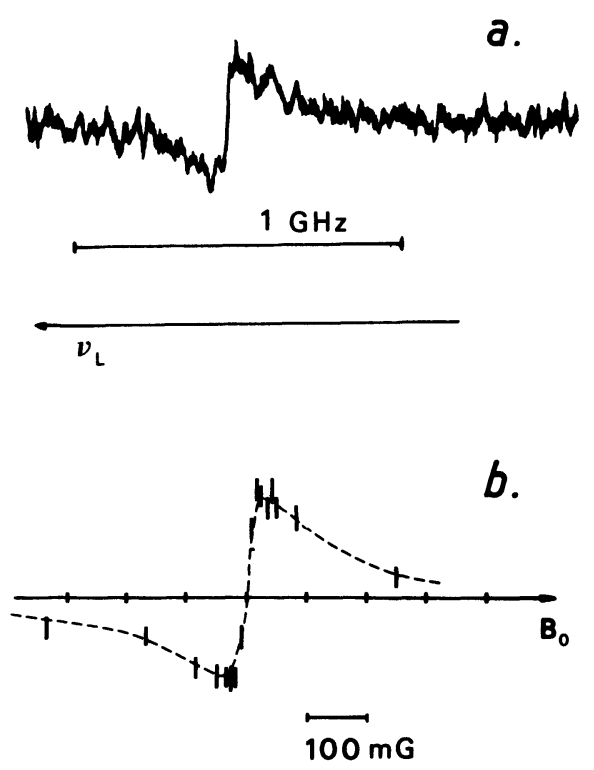

Fig. 7. - a) Signal d'orientation résultant du couplage de deux alignements (ici respectivement contenu dans le plan des faisceaux et perpendiculaire à lui) : l'amplitude du signal correspond à une modification d'environ $2 \times 10^{-5}$ de l'intensité du faisceau sonde. $b$ ) Variation de l'amplitude du signal précédent avec $B_{0}$ : les valeurs expérimentales sont représentées par des barres d'erreur; la courbe obtenue en reliant celles-ci entre elles a, comme prévu théoriquement, une allure de dispersion; le signal n'a pas été désaturé.

(a) Experimental signal due to an atomic orientation arising from the coupling between two alignments. $b$ ) Variation of the same signal as a function of the static magnetic field $B_{0}$.]

linéaires fixes pour le pompage (perpendiculaire l'une à l'autre dans le cas de la figure), et modulé à $100 \%$ les intensités des deux faisceaux $\left({ }^{10}\right)$. Les modulations de population parasites ainsi créées ne peuvent pas donner lieu à un effet d'orientation. La sonde a une intensité constante et sa polarisation est modulée alternativement $\sigma^{+}$et $\sigma^{-}$. L'amplitude du petit signal obtenu est d'environ $2 \times 10^{-5}$ de l'intensité du faisceau sonde. Le signal a, comme prévu théoriquement, un profil en dispersion $\left({ }^{11}\right)$.

Nous avons étudié les variations de l'amplitude de ce signal avec le champ magnétique $B_{0}$. Les points obtenus sont portés sur la figure $7 b$. Ces variations ont l'allure prévue théoriquement, tout en ayant encore des ailes plus larges que celles d'une courbe de disper-

$\left({ }^{10}\right)$ L'avantage de cette méthode est d'éviter que, par un léger déréglage de la biréfringence des cristaux électrooptiques, chaque faisceau de pompage ne crée une petite orientation modulée.

( $\left.{ }^{11}\right)$ Cette expérience est réalisée avec des polarisations linéaires fixes orthogonales entre elles (ce qui n'est pas la struation envisagée dans la partie 2); dans ce cas, la théorie prévoit que le signal varie avec le champ magnétique comme une fonction de dispersion de Lorentz de demi-largeur $\gamma / 2$.

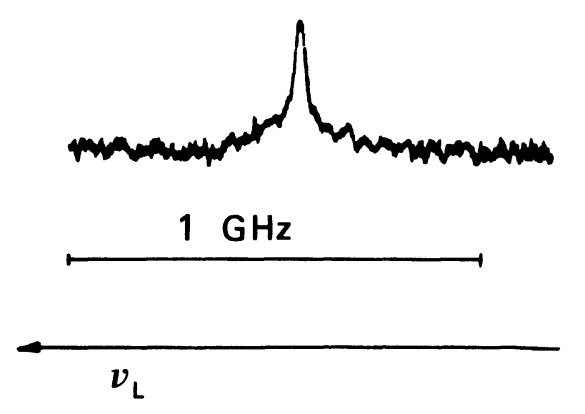

Fig. 8. - Signal d'orientation résultant du couplage d'une orientation avec un " trou de population ». La polarisation d'un des faisceaux de pompage est modulée alternativement $\sigma^{+}$et $\sigma^{-}$, tandis que l'autre faisceau, de polarisation linéaire fixe, est modulé à $100 \%$ en intensité. Ce signal, obtenu pour un champ fort (plus de $100 \mathrm{mG}$ ), est indépendant de la direction de polarisation du deuxième faisceau.

[Signal due to an atomic orientation obtained by coupling an orientation with a population hole. One pumping beam is polarization modulated $\left(\sigma^{+}, \sigma^{-}\right)$, the other has a fixed linear polarization but a modulated intensity.]

sion de Lorentz. Cependant, pour étudier cet effet, il ne nous a pas été possible d'atténuer les faisceaux de pompage de façon à désaturer le signal. Des effets d'ordres supérieurs sont certainement superposés ici à l'effet croisé proportionnel à $I_{1} I_{2}$.

- Les autres signaux que nous avons observés sont des signaux de "pompage-pompage», ayant donc un profil d'absorption avec la fréquence du laser. Nous nous contenterons ici de les citer, n'ayant pas pu en mener une étude détaillée :

- signal d'orientation résultant du couplage entre une orientation et un trou de population (effet en $\left[T_{0}^{(1)}, T_{0}^{(0)}\right]_{+}$qui est indépendant du champ magnétique); la figure 8 donne un exemple d'un tel signal;

- signal d'orientation résultant du couplage entre une orientation et un alignement (effet en $\left[T_{1}^{(1)}\right.$, $\left.T_{-1}^{(2)}\right]_{+}$et $\left[T_{-1}^{(1)}, T_{1}^{(2)}\right]_{+}$dont la variation en champ magnétique est paire);

- signal d'alignement résultant du couplage entre deux orientations (effet en $\left[T_{ \pm 1}^{(1)}, T_{ \pm 1}^{(1)}\right]_{+}$dont la variation en champ magnétique est paire).

4. Conclusion. - Dans cette expérience, l'excitation par deux faisceaux non colinéaires permet de sélectionner des atomes ayant deux composantes de leur vitesse déterminées. L'utilisation d'effets de pompage optique croisé permet d'observer une grande variété de signaux.

La généralisation de cette méthode à trois faisceaux de pompage non coplanaires serait possible à condition d'améliorer le rapport signal sur bruit que nous avons obtenu (20 au maximum). Un effet de saturation croisé permettrait alors de sélectionner dans la cellule les atomes quasi-immobiles (suppression des élargissements de raie introduits par la distorsion des fronts d'onde). Enfin, de nouvelles études de collisions 
pourraient être menées par ce type de méthode qui sélectionne plusieurs composantes de la vitesse des atomes : elles donneraient accès plus en détail aux différentes caractéristiques physiques des collisions.
Remerciements. - Les auteurs remercient vivement Monsieur J. P. Barrat pour de nombreuses suggestions utiles faites lors de la lecture du manuscrit.

\section{APPENDICE 1}

1. Position du problème. - On se propose de résoudre, à l'ordre 4 en champ électrique, les équations d'évolution de l'opérateur densité du système atomique. Les causes d'évolution du système sont l'interaction avec les deux ondes incidentes de fréquences $\Omega_{1}$ et $\Omega_{2}$, l'émission spontanée, l'évolution libre sous l'effet du hamiltonien atomique $H_{0}\left({ }^{12}\right)$, et enfin les processus de relaxation qui détruisent les observables de l'état fondamental (ou excité). On se place en représentation d'interaction par rapport à $H_{0}$; les équations d'évolution de $\tilde{\sigma}_{g}, \tilde{\sigma}_{\mathrm{e}}$ ef $\tilde{\sigma}_{\mathrm{eg}}$ s'écrivent [15] :

$$
\begin{aligned}
& \dot{\tilde{\sigma}}_{\mathrm{g}}=\frac{i}{\hbar}\left[\tilde{\mathfrak{D}}_{\mathrm{ge} 1} \tilde{\sigma}_{\mathrm{eg}} \varepsilon_{1}^{*} e^{i \Omega_{1} t}-\tilde{\sigma}_{\mathrm{ge}} \tilde{D}_{\mathrm{eg} 1} \varepsilon_{1} e^{-i \Omega_{1} t}\right]+i \operatorname{dem}(1 \rightarrow 2)+\tilde{\mathscr{G}}\left(\sigma_{\mathrm{e}}\right)-\gamma \tilde{\sigma}_{\mathrm{g}} \\
& \dot{\tilde{\sigma}}_{\mathrm{e}}=\frac{i}{\hbar}\left[\tilde{\mathfrak{D}}_{\mathrm{eg} 1} \tilde{\sigma}_{\mathrm{ge}} \varepsilon_{1} \mathrm{e}^{-i \Omega_{1} t}-\tilde{\sigma}_{\mathrm{eg}} \tilde{\mathfrak{D}}_{\mathrm{ge} 1} \varepsilon_{1}^{*} \mathrm{e}^{+i \Omega_{1} t}\right]+i \operatorname{dem}(1 \rightarrow 2)-\Gamma \tilde{\sigma}_{\mathrm{e}} \\
& \dot{\tilde{\sigma}}_{\mathrm{eg}}=\frac{i}{\hbar} \widetilde{\mathfrak{D}}_{\mathrm{eg} 1} \tilde{\sigma}_{\mathrm{g}} \varepsilon_{1} \mathrm{e}^{-i \Omega_{1 t}}+i \operatorname{dem}(1 \rightarrow 2)-\Gamma_{\mathrm{eg}} \tilde{\sigma}_{\mathrm{eg}}
\end{aligned}
$$

$\mathscr{D}_{\text {eg1 }}$ et $\mathscr{D}_{\mathrm{eg} 2}$ sont les éléments de matrice des opérateurs $\left(\mathbf{e}_{\lambda_{1}} \cdot \mathfrak{D}\right)$ et $\left(\mathbf{e}_{\lambda_{2}} \cdot \mathfrak{D}\right)$ entre les niveaux e et $\mathbf{g}(\mathfrak{D}$ est l'opérarateur dipolaire électrique); on a :

$$
\tilde{D}_{\text {eg1 }}=\mathrm{e}^{i H_{0 t}}\left(\mathbf{e}_{\lambda_{1}} \cdot \mathfrak{D}\right)_{\text {eg }} \mathrm{e}^{-i H_{0 t} t}=\mathscr{D}_{\text {eg1 }} \mathrm{e}^{i \Omega_{0 t} t} .
$$

Dans l'équation (A 1.3), on a négligé les éléments de matrice de $\sigma_{\mathrm{e}}$ devant ceux de $\sigma_{\mathrm{g}}$; ceci est justifié par le fait que les deux durées de vie des niveaux e et $\mathrm{g}$ sont très différentes.

$\Gamma$ et $\Gamma_{\mathrm{eg}}$ sont les taux de relaxation de $\sigma_{\mathrm{e}}$ et $\sigma_{\mathrm{eg}}$. On supposera la pression suffisamment faible pour que cette relaxation soit uniquement due au processus d'émission spontanée. $\mathcal{G}\left(\sigma_{\mathrm{e}}\right)$ décrit le transfert à partir du niveau $\mathrm{e}$ vers le niveau g par émission spontanée (sommation sur toutes les directions et toutes les polarisations du photon émis); $\mathcal{C}$ est un opérateur scalaire.

2. Calcul de $\tilde{\sigma}_{\mathrm{g}}$, à l'ordre 4 en champ électrique, en négligeant l'émission spontanée. - Nous allons, dans un premier temps, résoudre par itération les équations (A 1.1) et (A 1.3) en négligeant la retombée par émission spontanée, de façon à mettre en évidence les différents types de termes qui apparaissent dans $\tilde{\sigma}_{\mathrm{g}}$ à l'ordre 2 et à l'ordre 4 en champ électrique $\left({ }^{13}\right)$.

A l'ordre le plus bas en champ électrique (ordre 0), l'opérateur densité $\tilde{\sigma}_{\mathrm{g}}$ est isotrope :

$$
\text { (0) } \tilde{\sigma}_{\mathrm{g}}=\frac{P_{\mathrm{g}}}{2 J_{\mathrm{g}}+1} \text {. }
$$

A l'ordre 1, l'équation(A 1.3) s'écrit :

$$
{ }^{(1)} \tilde{\sigma}_{\mathrm{eg}}+\Gamma_{\mathrm{eg}}{ }^{(1)} \tilde{\sigma}_{\mathrm{eg}}=\frac{i}{\hbar\left(2 J_{\mathrm{g}}+1\right)}\left(\mathbf{e}_{\lambda_{1}} \cdot \boldsymbol{D}\right)_{\mathrm{eg}} \varepsilon_{1} \mathrm{e}^{i \Delta \Omega_{1 t} t}+\operatorname{idem}(1 \rightarrow 2)
$$

où l'on a posé $\Delta \Omega_{1}=\Omega_{0}-\Omega_{1}$.

Sa solution, pour des temps longs devant $\tau_{\mathrm{eg}}=\Gamma_{\mathrm{eg}}^{-1}$ est de la forme :

$$
{ }^{(1)} \tilde{\sigma}_{\mathrm{eg}}=\frac{i}{\hbar\left(2 J_{\mathrm{g}}+1\right)}\left(\mathbf{e}_{\lambda_{1}} \cdot \mathfrak{D}\right)_{\mathrm{eg}} \delta_{1} \frac{1}{\Gamma_{\mathrm{eg}}+i \Delta \Omega_{1}} \mathrm{e}^{i \Delta \Omega_{1} t}+\operatorname{idem}(1 \rightarrow 2) \text {. }
$$

(12) Pour plus de simplicité, on va supposer ici que le champ magnétique appliqué est nul.

$\left({ }^{13}\right)$ Dans cet appendice, ${ }^{(n)} \sigma$ représente un opérateur densité calculé à l'ordre $n$ en amplitude du champ électrique, contrairement à la notation utilisée dans le corps de l'article et dans l'appendice 2. 
En reportant cette expression dans (A 1.1) et en intégrant de la même façon, on obtient $\tilde{\sigma}_{\mathrm{g}}$ à l'ordre 2 en champ électrique.

$$
\text { (2) } \begin{aligned}
\tilde{\sigma}_{\mathrm{g}}=-\frac{\mathfrak{D}^{2} \varepsilon_{1}^{2}}{\hbar^{2}\left(2 J_{\mathrm{g}}+1\right)} \frac{2 \Gamma_{\mathrm{eg}}}{\gamma} \frac{A_{11}}{\Gamma_{\mathrm{eg}}^{2}+\Delta \Omega_{1}^{2}}+ & \operatorname{idem}(1 \rightarrow 2)-\frac{\mathfrak{D}^{2} \varepsilon_{1}^{*} \varepsilon_{2}}{\hbar^{2}\left(2 J_{\mathrm{g}}+1\right)} \frac{\mathrm{e}^{-i \Omega t}}{\gamma-i \Omega} \times \\
& \times A_{12}\left[\frac{1}{\Gamma_{\mathrm{eg}}+i \Delta \Omega_{2}}+\frac{1}{\Gamma_{\mathrm{eg}}-i \Delta \Omega_{1}}\right]+\text { herm. conj. }
\end{aligned}
$$

avec

$$
\begin{aligned}
\Omega & =\Omega_{2}-\Omega_{1} \\
A_{i j} & =\left(\mathbf{e}_{\lambda i}^{*} \cdot \mathbf{D}\right)_{\mathrm{ge}}\left(\mathbf{e}_{\lambda j} \cdot \mathbf{D}\right)_{\mathrm{eg}} \quad i, j=1,2 \\
\mathfrak{D}^{2} & =\left|\left\langle J_{\mathrm{g}}\|\boldsymbol{D}\| J_{\mathrm{e}}\right\rangle\right|^{2} .
\end{aligned}
$$

Dans cette expression, le premier terme décrit l'effet du pompage par le faisceau 1, le deuxième celui du pompage par le faisceau 2 : ces termes sont identiques à ceux qui apparaissent dans l'équation (10). Les deux termes supplémentaires correspondent au processus physique où le faisceau 2 (resp. 1) crée une cohérence optique que le faisceau 1 (resp. 2) transforme ensuite en population Zeeman dans le niveau métastable. Comme les atomes voient deux ondes de fréquences différentes $\Omega_{1}$ et $\Omega_{2}$, ces termes d'interférence oscillent à la fréquence $\Omega_{1}-\Omega_{2}$.

En reportant ces différents termes dans (A 1.3), on obtient les termes sources intervenant dans l'évolution de $^{(3)} \tilde{\sigma}_{\text {eg }}$. Certains évoluent aux fréquences $\Omega_{1}$ et $\Omega_{2}$, d'autres aux fréquences $\Omega_{1}-2 \Omega_{2}$ et $\Omega_{2}-2 \Omega_{1}$. Ces derniers, qui seront responsables de termes oscillants à ces mêmes fréquences, dans l'expression de ${ }^{(3)} \tilde{\sigma}_{\text {eg }}$ en régime stationnaire, ne pourront pas donner de termes sources statiques dans ${ }^{(4)} \dot{\tilde{\sigma}}_{\mathrm{g}}$. Nous n'en tiendrons donc pas compte, en ne gardant dans ${ }^{(3)} \dot{\tilde{\sigma}}_{\text {eg }}$ que les termes évoluant aux fréquences $\Omega_{1}$ ou $\Omega_{2}$. Ils sont au nombre de 6 :

$$
\left\{\begin{array}{l}
-i \frac{\mathfrak{D}^{2} \varepsilon_{1} \varepsilon_{1}^{2}}{\hbar^{3}\left(2 J_{\mathrm{g}}+1\right)} \frac{2 \Gamma_{\mathrm{eg}}}{\gamma} \frac{1}{\Gamma_{\mathrm{eg}}^{2}+\Delta \Omega_{1}^{2}} \widetilde{\mathfrak{D}}_{\mathrm{eg} 1} A_{11} \mathrm{e}^{-i \Omega_{1} t}+i \operatorname{dem}(1 \rightarrow 2) \\
-i \frac{\mathfrak{D}^{2} \varepsilon_{2} \varepsilon_{1}^{2}}{\hbar^{3}\left(2 J_{\mathrm{g}}+1\right)} \frac{2 \Gamma_{\mathrm{eg}}}{\gamma} \frac{1}{\Gamma_{\mathrm{eg}}^{2}+\Delta \Omega_{1}^{2}} \widetilde{\mathfrak{D}}_{\mathrm{eg} 2} A_{11} \mathrm{e}^{-i \Omega_{2} t}+i d e m(1 \leftrightarrow 2) \\
-i \frac{\mathfrak{D}^{2} \varepsilon_{2} \varepsilon_{1}^{2}}{\hbar^{3}\left(2 J_{\mathrm{g}}+1\right)} \frac{1}{\gamma-i \Omega}\left[\frac{1}{\Gamma_{\mathrm{eg}}+i \Delta \Omega_{2}}+\frac{1}{\Gamma_{\mathrm{eg}}-i \Delta \Omega_{1}}\right] \widetilde{\mathfrak{D}}_{\mathrm{eg} 1} A_{12} \mathrm{e}^{-i \Omega_{2} t}+i d e m(1 \leftrightarrow 2 \text { et } \Omega \leftrightarrow-\Omega) .
\end{array}\right.
$$

Ces termes s'intègrent de façon tout à fait analogue à ce qui a été fait pour ${ }^{(1)} \tilde{\sigma}_{\text {eg }}$. Ils sont ensuite reportés dans (A 2.1) et l'on peut alors distinguer trois sortes de termes statiques dans ${ }^{(4)} \dot{\tilde{\sigma}}_{\mathrm{g}}+\gamma^{(4)} \tilde{\sigma}_{\mathrm{g}}$.

2.1 TeRmes À 1 SeUl faisceau. - Il y en a un pour chaque faisceau, de la forme :

$$
\frac{D^{4} \varepsilon_{1}^{2} \varepsilon_{1}^{2}}{\hbar^{4}\left(2 J_{\mathrm{g}}+1\right)} \frac{2 \Gamma_{\mathrm{eg}}}{\gamma} \frac{1}{\Gamma_{\mathrm{eg}}^{2}+\Delta \Omega_{1}^{2}} \times \frac{1}{\Gamma_{\mathrm{eg}}+i \Delta \Omega_{1}} A_{11}^{2}+\text { herm. conj. }
$$

Leur intégration conduit dans ${ }^{(4)} \tilde{\sigma}_{\mathrm{g}}$ à deux termes du deuxième ordre en intensité, l'un en $I_{1}^{2}$ l'autre en $I_{2}^{2}$, correspondant à la saturation du pompage optique par chacun des faisceaux :

$$
\text { (4) } \tilde{\sigma}_{\mathrm{g}}=\frac{4 \mathfrak{D}^{4} I_{1}^{2}}{\hbar^{4}\left(2 J_{\mathrm{g}}+1\right) \gamma^{2}} \frac{\Gamma_{\mathrm{eg}}^{2}}{\left(\Gamma_{\mathrm{eg}}^{2}+\Delta \Omega_{1}^{2}\right)^{2}} A_{11}^{2}+\operatorname{idem}(1 \rightarrow 2) \text {. }
$$

2.2 TeRMeS CROISÉS EN $A_{11} A_{22}$. - Les termes sources correspondants s'écrivent :

$$
\frac{D^{4} \varepsilon_{2}^{2} \varepsilon_{1}^{2}}{\hbar^{4}\left(2 J_{\mathrm{g}}+1\right)} \frac{2 \Gamma_{\mathrm{eg}}}{\gamma} \frac{1}{\Gamma_{\mathrm{eg}}^{2}+\Delta \Omega_{1}^{2}} \times \frac{1}{\Gamma_{\mathrm{eg}}+i \Delta \Omega_{2}} A_{22} A_{11}+\text { herm. conj. }+\operatorname{idem}(1 \leftrightarrow 2) .
$$

Leur intégration donne dans ${ }^{(4)} \tilde{\sigma}_{\mathrm{g}}$ la contribution :

$$
\text { (4) } \tilde{\sigma}_{\mathrm{g}}=\frac{2 D^{4} I_{1} I_{2}}{\hbar^{4}\left(2 J_{\mathrm{g}}+1\right) \gamma^{2}} \frac{\Gamma_{\mathrm{eg}}}{\Gamma_{\mathrm{eg}}^{2}+\Delta \Omega_{1}^{2}}\left[\frac{\Gamma_{\mathrm{eg}}}{\Gamma_{\mathrm{eg}}^{2}+\Delta \Omega_{2}^{2}}\left[A_{22}, A_{11}\right]_{+}-\frac{i \Delta \Omega_{2}}{\Gamma_{\mathrm{eg}}^{2}+\Delta \Omega_{2}^{2}}\left[A_{22}, A_{11}\right]\right]+\operatorname{idem}(1 \leftrightarrow 2) \text {. }
$$

On retrouve ici les termes croisés donnés dans(11) et (12). 
2.3 TeRMES CROISÉS EN $A_{21} A_{12}$. - Les termes sources correspondants s'écrivent :

$$
\begin{array}{r}
\frac{D^{4} \varepsilon_{2}^{2} \varepsilon_{1}^{2}}{\hbar^{4}\left(2 J_{\mathrm{g}}+1\right)} \frac{1}{\gamma-i \Omega}\left[\frac{1}{\Gamma_{\mathrm{eg}}+i \Delta \Omega_{2}}+\frac{1}{\Gamma_{\mathrm{eg}}-i \Delta \Omega_{1}}\right] \times \frac{1}{\Gamma_{\mathrm{eg}}+i \Delta \Omega_{2}} A_{21} A_{12}+ \\
\quad \quad+\text { herm. conj. }+ \text { idem }(1 \leftrightarrow 2 \text { et } \Omega \leftrightarrow-\Omega) .
\end{array}
$$

Ils proviennent des termes d'interférence dans ${ }^{(2)} \tilde{\sigma}_{\mathrm{g}}$. Leur intégration donne dans ${ }^{(4)} \tilde{\sigma}_{\mathrm{g}}$ la contribution :

$$
\text { (4) } \begin{aligned}
\tilde{\sigma}_{\mathrm{g}}=\frac{D^{4} I_{1} I_{2}}{\hbar^{4}\left(2 J_{\mathrm{g}}+1\right) \gamma(\gamma-i \Omega)}\left[\frac{1}{\Gamma_{\mathrm{eg}}+i \Delta \Omega_{2}}+\frac{1}{\Gamma_{\mathrm{eg}}-i \Delta \Omega_{1}}\right] \times \frac{1}{\Gamma_{\mathrm{eg}}+i \Delta \Omega_{2}} A_{21} A_{12}+ \\
\quad+\text { herm. conj. }+i \operatorname{idem}(1 \leftrightarrow 2 \text { et } \Omega \leftrightarrow-\Omega) .
\end{aligned}
$$

3. Contribution des termes d'interférence au signal détecté. - Dans l'opérateur densité ${ }^{(4)} \tilde{\sigma}_{\mathrm{g}}$ apparaissent deux sortes de termes croisés en $I_{1} I_{2}$. Les termes en $A_{11} A_{22}$ proviennent de termes statiques dans ${ }^{(2)} \tilde{\sigma}_{g}$, tandis que les termes en $A_{21} A_{12}$ proviennent de termes d'interférence, oscillant à la fréquence $\Omega=\Omega_{2}-\Omega_{1}$, associés à un effet de battement entre les fréquences $\Omega_{1}$ et $\Omega_{2}$ vues par un atome de vitesse $\mathbf{v}$ donnée. Le niveau $\mathrm{g}$ a un faible taux de relaxation $\gamma$, et la réponse du système atomique dans ce niveau à l'excitation oscillante de fréquence $\Omega$ n'est appréciable que dans un petit domaine de fréquence centré en $\Omega=0$ et de largeur $\gamma$. La fréquence $\Omega$ étant directement reliée à la vitesse des atomes, c'est seulement pour les atomes appartenant au petit domaine de vitesse correspondant de largeur $\gamma / k$, que les termes en $A_{12} A_{21}$ dans ${ }^{(4)} \tilde{\sigma}_{\mathrm{g}}$ sont importants.

Nous allons montrer dans ce paragraphe que lorsqu'on calcule le signal dû aux termes croisés en $I_{1} I_{2}$, ce qui se fait par intégration sur les composantes $v_{x}$ et $v_{z}$ de la vitesse des atomes, la contribution des termes en $A_{21} A_{12}$ est négligeable devant celle des termes en $A_{11} A_{22}$ car le nombre d'atomes participant au signal est beaucoup plus petit (dans un rapport $\Gamma_{\mathrm{eg}} / \gamma$ ).

Pour discuter de la grandeur des signaux en question, il est utile de transformer l'expression donnée en (A 1.5). Pour cela on remarque que :

$$
\frac{1}{\gamma \mp i \Omega}=\frac{\gamma}{\gamma^{2}+\Omega^{2}} \pm \frac{i \Omega}{\gamma^{2}+\Omega^{2}}
$$

On peut alors réécrire les termes croisés en $A_{21} A_{12}$ ou $A_{12} A_{21}$ sous la forme suivante :

$$
\text { (4) } \begin{aligned}
\tilde{\sigma}_{\mathrm{g}}= & \frac{2 D^{4} I_{1} I_{2}}{\gamma^{2}+\Omega^{2}} \times \\
& \times\left\{\left[\frac{\Gamma_{\mathrm{eg}}^{2}-\Delta \Omega_{2}^{2}}{\left(\Gamma_{\mathrm{eg}}^{2}+\Delta \Omega_{2}^{2}\right)^{2}} A_{21} A_{12}+\frac{\Gamma_{\mathrm{eg}}^{2}-\Delta \Omega_{1}^{2}}{\left(\Gamma_{\mathrm{eg}}^{2}+\Delta \Omega_{1}^{2}\right)^{2}} A_{12} A_{21}+\frac{\Gamma_{\mathrm{eg}}^{2}+\Delta \Omega_{1} \Delta \Omega_{2}}{\left(\Gamma_{\mathrm{eg}}^{2}+\Delta \Omega_{1}^{2}\right)\left(\Gamma_{\mathrm{eg}}^{2}+\Delta \Omega_{2}^{2}\right)}\left[A_{21}, A_{12}\right]_{+}\right]\right. \\
& \left.+\frac{\Omega}{\gamma}\left[\frac{2 \Gamma_{\mathrm{eg}} \Delta \Omega_{2}}{\left(\Gamma_{\mathrm{eg}}^{2}+\Delta \Omega_{2}^{2}\right)^{2}} A_{21} A_{12}-\frac{2 \Gamma_{\mathrm{eg}} \Delta \Omega_{1}}{\left(\Gamma_{\mathrm{eg}}^{2}+\Delta \Omega_{1}^{2}\right)^{2}} A_{12} A_{21}-\frac{\Gamma_{\mathrm{eg}} \Omega}{\left(\Gamma_{\mathrm{eg}}^{2}+\Delta \Omega_{1}^{2}\right)\left(\Gamma_{\mathrm{eg}}^{2}+\Delta \Omega_{2}^{2}\right)}\left[A_{21}, A_{12}\right]_{+}\right]\right\} .
\end{aligned}
$$

Chacun des termes contenus dans cette expression contient le produit de deux courbes de Lorentz (en absorption ou en dispersion) de variables $\Delta \Omega_{1}$ ou $\Delta \Omega_{2}$ et de demi-largeur $\Gamma_{\text {eg. }}$. Ils sont donc d'une forme analogue à celle des termes croisés en $A_{11} A_{22}$; mais ils sont maintenant précédés, soit du coefficient

$$
\frac{1}{\gamma} \frac{\gamma}{\gamma^{2}+\Omega^{2}}
$$

soit du coefficient

$$
\frac{1}{\gamma} \frac{\Omega}{\gamma^{2}+\Omega^{2}}
$$

alors que les autres étaient simplement multipliés par $1 / \gamma^{2}$.

Ces termes doivent maintenant être sommés sur les vitesses des atomes après avoir été multipliés par la fonction de détection comme il est fait en 2.4.2. Il est alors commode d'introduire le vecteur $\mathbf{K}$ défini par :

$$
\mathbf{K}=\mathbf{k}_{1}-\mathbf{k}_{\mathbf{2}}
$$

et l'on a :

$$
\Omega=\mathbf{K} \cdot \mathbf{v} .
$$


Les coefficients $\frac{\gamma}{\gamma^{2}+\Omega^{2}}$ et $\frac{\Omega}{\gamma^{2}+\Omega^{2}}$ dans les intégrales sont des fonctions de Lorentz qui ne sont non nulles que dans un domaine de vitesse correspondant à $\left|v_{\mathbf{u}}\right| \lesssim \gamma / K$ où $v_{\mathbf{u}}$ est la projection de $\mathbf{v}$ sur $\mathbf{K}$. Ce domaine est beaucoup plus étroit que les domaines où les fonctions de pompage et de détection sont non nulles (respectivement $\left|v_{1}\right| \lesssim \Gamma_{\text {eg }} / k,\left|v_{2}\right| \lesssim \Gamma_{\text {eg }} / k$ et $\left.\left|v_{z}\right| \lesssim \Gamma_{\text {eg }} / k\right)$. Si les deux faisceaux ne sont pas parallèles $(K \neq 0)$, ces coefficients supplémentaires introduisent une nouvelle sélection sur les vitesses nettement plus sévère que celle des signaux calculés en 2.4. Les intégrales correspondantes vont donc être plus petites d'un facteur de l'ordre de $\Gamma_{\text {eg }} / \gamma$.

4. Contribution due à l'émission spontanée. - Un calcul tout à fait analogue au précédent peut être fait de façon à mettre en évidence dans ${ }^{(2)} \tilde{\sigma}_{\mathrm{g}}$ et ${ }^{(4)} \tilde{\sigma}_{\mathrm{g}}$ les termes dus à l'émission spontanée.

On montre facilement que l'opérateur densité ${ }^{(2)} \tilde{\sigma}_{\mathrm{e}}$ créé à l'ordre 2 en champ électrique s'écrit :

$$
\text { (2) } \begin{aligned}
\tilde{\sigma}_{\mathrm{e}}=\left\{\frac{D^{2} \varepsilon_{1}^{2}}{\hbar^{2}\left(2 J_{\mathrm{g}}+1\right)} \frac{2 \Gamma_{\mathrm{eg}}}{\Gamma} \frac{E_{11}}{\Gamma_{\mathrm{eg}}^{2}+\Delta \Omega_{1}^{2}}+\operatorname{idem}(1 \rightarrow 2)\right\}+ \\
\quad+\left\{\frac{\mathfrak{D}^{2} \varepsilon_{1}^{*} \varepsilon_{2}}{\hbar^{2}\left(2 J_{\mathrm{g}}+1\right)} \frac{\mathrm{e}^{-i \Omega t}}{\Gamma-i \Omega} E_{21}\left[\frac{1}{\Gamma_{\mathrm{eg}}+i \Delta \Omega_{2}}+\frac{1}{\Gamma_{\mathrm{eg}}-i \Delta \Omega_{1}}\right]+\text { herm. conj. }\right\}
\end{aligned}
$$

où

$$
E_{i j}=\left(\mathbf{e}_{\lambda i} \cdot \mathbf{D}\right)_{\mathrm{eg}}\left(\mathbf{e}_{\lambda j}^{*} \cdot \mathbf{D}\right)_{\mathrm{ge}} .
$$

Au deuxième ordre en champ électrique, l'émission spontanée va donc ramener dans ${ }^{(2)} \tilde{\sigma}_{\mathrm{g}}$, en plus des deux termes statiques dus à chacun des faisceaux, deux termes oscillants de la forme :

$$
\frac{D^{2} \varepsilon_{1}^{*} \varepsilon_{2}}{\hbar^{2}\left(2 J_{\mathrm{g}}+1\right)} \frac{\mathrm{e}^{-i \Omega t}}{\gamma-i \Omega} \frac{\mathscr{C}\left(E_{21}\right)}{\Gamma-i \Omega}\left[\frac{1}{\Gamma_{\mathrm{eg}}+i \Delta \Omega_{2}}+\frac{1}{\Gamma_{\mathrm{eg}}-i \Delta \Omega_{1}}\right]+\text { herm. conj. }
$$

Ces termes sont analogues aux termes oscillants de (A 1.4); on passe des uns aux autres en remplaçant $-A_{12}$ $\operatorname{par} \frac{\mathcal{C}\left(E_{21}\right)}{\Gamma-i \Omega}$. Remarquons que $\mathcal{C}\left(E_{21}\right)$ contient le coefficient $\Gamma_{\mathrm{r}}$ qui est de l'ordre de grandeur de $\Gamma$. Au $4^{\mathrm{e}}$ ordre en champ électrique, ces termes sont responsables de termes statiques dans ${ }^{(4)} \tilde{\sigma}_{\mathrm{g}}$ soit directement par un effet de dépopulation soit par un effet de pompage (retombée incluse). Les deux types de termes correspondants dans (4) $\tilde{\sigma}_{\mathrm{g}}$ s'écrivent :

$$
\begin{aligned}
-\frac{D^{4} I_{1} I_{2}}{\hbar^{4}\left(2 J_{\mathrm{g}}+1\right) \gamma(\gamma-i \Omega)} A_{21} \frac{\mathfrak{C}\left(E_{21}\right)}{\Gamma-i \Omega}\left[\frac{1}{\Gamma_{\mathrm{eg}}+i \Delta \Omega_{2}}+\frac{1}{\Gamma_{\mathrm{eg}}-i \Delta \Omega_{1}}\right] \times \frac{1}{\Gamma_{\mathrm{eg}}+i \Delta \Omega_{2}}+ \\
+ \text { herm. conj. }+ \text { idem }(1 \leftrightarrow 2, \Omega \leftrightarrow-\Omega),
\end{aligned}
$$

et :

$$
\begin{array}{r}
+\frac{D^{4} I_{1} I_{2}}{\hbar^{4}\left(2 J_{\mathrm{g}}+1\right) \gamma(\gamma-i \Omega)} \frac{\mathfrak{C}\left[E_{12} \cdot \mathcal{G}\left(E_{21}\right)\right]}{\Gamma(\Gamma-i \Omega)}\left[\frac{1}{\Gamma_{\mathrm{eg}}+i \Delta \Omega_{2}}+\frac{1}{\Gamma_{\mathrm{eg}}-i \Delta \Omega_{1}}\right] \times \frac{1}{\Gamma_{\mathrm{eg}}+i \Delta \Omega_{2}}+ \\
\quad+\text { herm. conj. }+i d e m(1 \leftrightarrow 2, \Omega \leftrightarrow-\Omega) .
\end{array}
$$

La dépendance en fréquence de ces termes est analogue à celle des termes de (A 1.5) avec cependant le facteur supplémentaire $\frac{1}{\Gamma-i \Omega}$. Leur contribution au signal détecté, qui s'obtient en intégrant sur les vitesses des atomes est du même ordre de grandeur que celle des termes de (A 1.5). En effet, le domaine d'intégration où le signal est appréciable est, comme nous l'avons vu dans le paragraphe précédent, de l'ordre de $\gamma / K$, et dans ce domaine la fonction $\frac{1}{\Gamma-i \Omega}$ peut être approximée par $1 / \Gamma$.

5. Interaction avec le faisceau sonde. - Nous avons admis dans 2.4 que les effets de cohérence entre les faisceaux de pompage et le faisceau sonde apportaient au signal une contribution négligeable, et nous n'avons donc considéré l'interaction du système atomique avec le champ électrique $\varepsilon_{\mathrm{S}}$ de la sonde qu'après la préparation du système par les deux faisceaux de pompage. En toute rigueur, il faudrait tenir compte également des processus où le système interagit avec le champ $\varepsilon_{S}$ entre deux interactions avec les champs $\varepsilon_{1}$ et $\varepsilon_{2}$, mais on montre facilement que ces autres processus peuvent être négligés. 
Considérons par exemple le processus où le système atomique interagit d'abord deux fois avec le champ $\varepsilon_{1}$, de façon que l'opérateur densité intermédiaire ${ }^{(2)} \tilde{\sigma}_{\mathrm{g}}$ soit statique, puis avec le champ $\varepsilon_{\mathrm{s}}$, puis deux fois avec le champ $\varepsilon_{2}$. L'opérateur densité ${ }^{(4)} \tilde{\sigma}_{\mathrm{g}}$ intermédiaire est oscillant à la fréquence $\Omega_{2}-\Omega_{\mathrm{S}}$. La cohérence optique créée à l'ordre 5 et évoluant à la fréquence $-\Omega_{\mathrm{S}}$ est donc pondérée par un facteur $\frac{1}{\gamma+i\left(\Omega_{2}-\Omega_{\mathrm{S}}\right)}$. Pour une raison tout à fait identique à celle qui a été vue dans les paragraphes précédents dans le cas de l'interaction cohérente avec les deux faisceaux de pompage, la présence de ce facteur dans l'intégration sur les vitesses des atomes va diminuer la contribution de ce processus d'un facteur $\Gamma_{\text {eg }} / \gamma$ au moins (on a supposé que le faisceau sonde n'est parallèle à aucun des faisceaux de pompage). Un raisonnement similaire peut être fait avec les autres processus.

En résumé, et comme nous l'avons admis dans le $\S 2$, le fait que les trois faisceaux utilisés issus du même laser ne sont pas 2 à 2 colinéaires permet de n'envisager que le processus où le système atomique subit un cycle de pompage optique par un faisceau de pompage, puis un deuxième cycle par l'autre, puis enfin est sondé par le faisceau de détection.

\section{APPENDICE 2}

Les expériences que nous avons réalisées ont été faites avec une cellule contenant 10 mtorr de néon pur. Bien que cette pression soit faible, on sait, par des expériences de pompage optique sélectif en vitesse à un seul faisceau [7], que l'effet des collisions n'est pas totalement négligeable dans ces conditions, et qu'il modifie le profil des signaux obtenus. Nous avons donc repris le calcul de ${ }^{(2)} \sigma_{\mathrm{g}}$ de façon à prévoir qualitativement l'effet des collisions dans le niveau métastable sur les signaux de pompage optique croisé à deux faisceaux : on montre en particulier que les collisions conservent la parité des signaux par rapport à $\Omega_{\mathrm{L}}-\Omega_{0}$ et par rapport à $\omega_{0}$.

1. Evolution de $\sigma_{\mathrm{g}}$ sous l'effet des collisions. - Nous n'avons dans le $\S 2$ tenu compte des collisions subies par les atomes métastables, que par l'intermédiaire du terme de relaxation $-\gamma \sigma_{\mathrm{g}}$ dans l'évolution de l'opérateur densité. La relaxation dans le niveau g peut avoir différentes causes physiques : processus radiatif, diffusion des atomes hors des faisceaux de pompage, collisions, etc... Le taux de relaxation $\gamma$ peut donc être écrit sous la forme :

$$
\gamma=\gamma_{0}+\gamma_{c}
$$

où l'on a isolé le taux de relaxation $\gamma_{c}$ dû aux collisions avec les autres atomes $\left({ }^{14}\right)$. A priori, $\gamma_{c}$ et $\gamma_{0}$ sont des fonctions de la vitesse $\mathbf{v}$, ce qui fait que la matrice densité $\sigma_{\mathrm{g}}$ dépend de $\mathbf{v}$ non seulement par l'intermédiaire des fréquences $\Omega_{1}$ et $\Omega_{2}$, mais également par l'intermédiaire du taux de relaxation $\gamma$.

La description utilisée jusqu'ici serait suffisante si les collisions détruisaient totalement les observables créées par le pompage. Ceci est bien évidemment faux pour la population; c'est faux également pour l'orientation et l'alignement dans les collisions $\mathrm{Ne}^{*}$ - $\mathrm{He}$, et dans une moindre mesure dans les collisions $\mathrm{Ne}^{*}$ - $\mathrm{Ne}$, comme le montrent différentes expériences de pompage optique sélectif en vitesses [2], [7].

Il convient donc d'ajouter au terme de relaxation écrit précédemment, qui est un " terme de départ ", un " terme de retour » correspondant aux observables ramenées par collisions à partir des autres classes de vitesses vers la classe de vitesse considérée. Ce terme supplémentaire, introduit phénoménologiquement dans [11], fait intervenir un noyau de collisions de la forme :

$$
K^{(k)}\left(\mathbf{v}, \mathbf{v}^{\prime}\right)=P\left(\mathbf{v}, \mathbf{v}^{\prime}\right) \xi^{(k)}\left(\mathbf{v}, \mathbf{v}^{\prime}\right) .
$$

$P\left(\mathbf{v}, \mathbf{v}^{\prime}\right)$ est la probabilité par unité de temps pour qu'un atome soit transféré par collision de la vitesse $\mathbf{v}$ à la vitesse $\mathbf{v}^{\prime}$;

$\xi^{(k)}\left(\mathbf{v}, \mathbf{v}^{\prime}\right)$ est le paramètre de conservation de l'observable considérée lors de la collision; il dépend de l'ordre tensoriel $k$ de cette observable; on a toujours :

$$
0 \leqslant \xi \leqslant 1 .
$$

Nous admettrons que, pour une classe d'atomes de vitesse $\mathbf{v}$ donnée, le taux de relaxation par collisions des différentes observables, n'est rien d'autre que le taux de départ de la population :

$$
\gamma_{c}(\mathbf{v})=\iint_{-\infty}^{+\infty} \mathrm{d}^{2} v^{\prime} P\left(\mathbf{v}, \mathbf{v}^{\prime}\right) .
$$

$\left({ }^{14}\right)$ Dans les conditions expérimentales où nous nous sommes placés ( $p=10$ mtorr et faisceaux de pompage étendus), on a : $\gamma_{\mathrm{c}} \gg \gamma_{0}$. 
Ceci revient à dire qu'une collision qui change l'orientation ou l'alignement des atomes, change également leur vitesse : $\gamma_{\mathrm{c}}$ comme $\gamma_{0}$. sont indépendants de $k$.

$\gamma(v)$ étant une fonction qui ne varie pas très rapidement avec la vitesse, nous l'approximerons dans la suite à une constante.

Comme dans la référence [11], nous écrirons donc l'évolution de l'opérateur densité sous l'effet des collisions sous la forme :

$$
\left.\frac{\mathrm{d} \sigma_{\mathrm{g}}(\mathbf{v})}{\mathrm{d} t}\right|_{\mathrm{coll} .}=-\gamma \sigma_{\mathrm{g}}(\mathbf{v})+\iint_{-\infty}^{+\infty} \mathrm{d}^{2} \mathbf{v}^{\prime} \sum_{k, q} K^{(k)}\left(\mathbf{v}^{\prime}, \mathbf{v}\right) \sigma_{\mathrm{gq}}^{(k)}\left(\mathbf{v}^{\prime}\right) T_{q}^{(k)}
$$

où nous avons négligé tous les couplages par collision entre observables de symétries différentes $\left(k \neq k^{\prime}\right.$ ou $\left.q \neq q^{\prime}\right)$.

$\mathbf{v}$ et $\mathbf{v}^{\prime}$ sont des vecteurs vitesses à deux dimensions dans le plan $z \mathrm{O} x$, la composante $v_{y}$ ne jouant aucun rôle dans notre problème.

2. Calcul de ${ }^{(2)} \boldsymbol{\sigma}_{\mathrm{g}}$ - - On peut reprendre, avec ce nouveau terme d'évolution, le calcul des effets de pompage croisés à l'ordre le plus bas, dans l'opérateur densité $\sigma_{\mathrm{g}}$.

A l'ordre 1 en intensité cet opérateur densité, que nous noterons ${ }^{(1)} \sigma_{\mathbf{g}}(\mathbf{v})$ pour une classe de vitesse $\mathbf{v}$ donnée, peut être développé en série par rapport au nombre de collisions $n$ subies par l'atome après un premier cycle de pompage :

$$
{ }^{(1)} \sigma_{\mathrm{g}}(\mathbf{v})=\sum_{n=0}^{\infty}{ }_{n}^{(1)} \sigma_{\mathrm{g}}(\mathbf{v}) \text {. }
$$

Il en est de même pour les composantes ${ }^{(1)} \sigma_{\mathrm{gq}}^{(k)}$ correspondantes :

$$
{ }^{(1)} \sigma_{\mathrm{gq}}^{(k)}(\mathbf{v})=\sum_{n=0}^{\infty}{ }^{(1)} \sigma_{\mathrm{gq}}^{(k)}(\mathbf{v}) \text {. }
$$

On peut résoudre les équations d'évolution de ${ }^{(1)} \sigma_{\mathrm{g}}(\mathrm{v})$ par itération par rapport à $n$ (on suppose que les collisions n'ont pas le temps de se produire pendant la durée de vie de l'état excité). On obtient, en se limitant à un cycle de pompage optique par le faisceau 1 :

$$
{ }_{0}^{(1)} \sigma_{\mathrm{gq}}^{\left(k_{1}\right)}(\mathbf{v})=\frac{\Gamma_{1}^{\prime}(\mathbf{v})}{2 J_{\mathrm{g}}+1} \frac{\mathcal{B}_{k_{1}}\left(J_{\mathrm{g}}, J_{\mathrm{e}}\right) \varphi_{q_{1}}^{\left(k_{1}\right)}\left(\mathbf{e}_{\lambda_{1}}\right)}{i q_{1} \omega_{0}+\gamma}
$$

expression qui a déjà été calculée dans 2.3 .3 , et

$$
{ }_{n}^{(1)} \sigma_{g q_{1}}^{\left(k_{1}\right)}(\mathbf{v})=\iint_{-\infty}^{+\infty} \mathrm{d}^{2} \mathbf{v}^{\prime} \frac{K^{\left(k_{1}\right)}\left(\mathbf{v}^{\prime}, \mathbf{v}\right)_{n-1}^{(1)} \sigma_{g q_{1}}^{\left(k_{1}\right)}\left(\mathbf{v}^{\prime}\right)}{i q_{1} \omega_{0}+\gamma}
$$

Pour obtenir l'opérateur densité à l'ordre 2 en intensité, que nous noterons ${ }^{(2)} \sigma_{\mathrm{g}}(\mathbf{v})$, on reporte l'expression totale de ${ }^{(1)} \sigma_{g}(v)$ dans l'équation d'évolution de ${ }^{(2)} \sigma_{g}(v)$. Comme précédemment, il est commode de développer ${ }^{(2)} \sigma_{\mathrm{g}}(\mathbf{v})$ en série par rapport au nombre de collisions $p$ subies par l'atome après le deuxième cycle de pompage optique :

et

$$
{ }^{(2)} \sigma_{\mathrm{g}}(\mathbf{v})=\sum_{p=0}^{\infty}{ }_{p}^{(2)} \sigma_{\mathrm{g}}(\mathbf{v})
$$

$$
{ }^{(2)} \sigma_{\mathrm{gQ}}^{(K)}(\mathbf{v})=\sum_{p=0}^{\infty}{ }_{\dot{p}}^{(2)} \sigma_{\mathrm{gQ}}^{(K)}(\mathbf{v})
$$

L'équation d'évolution de ${ }^{(2)} \sigma_{\mathrm{g}}(\mathrm{v})$ peut alors être résolue par itération; nous nous limiterons aux termes de pompage croisés dus à l'action du faisceau 1 puis à celle du faisceau 2. On obtient :

$$
{ }_{0}^{(2)} \sigma_{\mathrm{gQ}}^{(K)}(\mathbf{v})=\frac{\Gamma_{2}^{\prime}}{i Q \omega_{0}+\gamma} \sum_{\substack{k_{1} q_{1} \\ k_{2} q_{2}}} C_{\mathrm{p}}\left(k_{1}, k_{2}, K\right) \varphi_{q_{2}}^{\left(k_{2}\right)}\left(\mathbf{e}_{\lambda_{2}}\right)^{(1)} \sigma_{\mathrm{g} q_{1}}^{\left(k_{1}\right)}(\mathbf{v})\left\langle k_{1} k_{2} q_{1} q_{2} \mid K Q\right\rangle
$$


pour les termes de " pompage-pompage",

$$
\underset{0}{(2)} \sigma_{\mathrm{gQ}}^{(K)}(\mathbf{v})=-\frac{2 i \Delta E_{2}^{\prime}}{i Q \omega_{0}+\gamma} \sum_{\substack{k_{1} q_{1} \\ k_{2 q_{2}}}} C_{\mathrm{d}}\left(k_{1}, k_{2}, K\right) \varphi_{q_{2}}^{\left(k_{2}\right)}\left(\mathbf{e}_{\lambda_{2}}\right)^{(1)} \sigma_{\mathbf{g q}_{1}}^{\left(k_{1}\right)}(\mathbf{v})\left\langle k_{1} k_{2} q_{1} q_{2} \mid K Q\right\rangle
$$

pour les termes de " pompage-déplacement », et

$$
{ }_{p}^{(2)} \sigma_{\mathrm{gQ}}^{(K)}(\mathbf{v})=\iint_{-\infty}^{+\infty} \mathrm{d}^{2} \mathbf{v}^{\prime} \frac{K^{(K)}\left(\mathbf{v}^{\prime}, \mathbf{v}\right)_{p-1}^{(2)} \sigma_{\mathrm{gQ}}^{(K)}\left(\mathbf{v}^{\prime}\right)}{i Q \omega_{0}+\gamma}
$$

Chacun des ${ }_{p}^{(2)} \sigma_{g Q}^{(K)}(\mathbf{v})$ est, par l'intermédiaire de ${ }^{(1)} \sigma_{q_{1}}^{\left(k_{1}\right)}$ une somme sur $n ;{ }^{(2)} \sigma_{g Q}^{(K)}$ se présente donc comme une double somme sur $n$ et sur $p$ de termes croisés ${ }_{n, p}^{(2)} \sigma_{\mathrm{gQ}}^{\left(K_{1}\right)}(\mathrm{v})$ correspondant à un cycle de pompage par le faisceau 1 suivi de $n$ collisions, puis un cycle de pompage par le faisceau 2 suivi de $p$ collisions. Chacun de ces termes croisés donne lieu à un signal, lorsqu'on détecte une observable proportionnelle à $T_{Q}^{(K)}$, qui est, après intégration sur toutes les classes de vitesse, de la forme :

$$
\begin{gathered}
S(\delta) \propto \iint_{-\infty}^{+\infty} \mathrm{d}^{2} \mathbf{v} \mathfrak{D}(\delta, \mathbf{v}) \underset{n, p}{(2)} \sigma_{Q}^{(K)}(\delta, \mathbf{v}) \\
\delta=\Omega_{\mathrm{L}}-\Omega_{0} \quad \text { et } \quad \mathcal{D}(\delta, \mathbf{v})=\frac{\Gamma_{\mathrm{eg}}}{\left(\delta-\mathbf{k}_{\mathrm{d}} \cdot \mathbf{v}\right)^{2}+\Gamma_{\mathrm{eg}}^{2}} \text { est la fonction de détection }
\end{gathered}
$$

3. Comportement en fréquence des signaux induits par les collisions. - Dans chacun des termes ${ }_{n, p}^{(2)} \sigma_{\mathrm{gQ}}^{(\mathrm{K})}(\delta, \mathbf{v})$ la dépendance en vitesse et en fréquence provient uniquement des coefficients $\Gamma^{\prime}(\delta, \mathbf{v})$ et $\Delta E^{\prime}(\delta, \mathbf{v})$ ainsi que des intégrales de collisions. Nous allons montrer que ces dernières ne modifient pas la symétrie, par rapport à $\delta$, du signal détecté.

Prenons l'exemple d'un signal de "pompage-déplacement». A l'ordre 1 en intensité, et en l'absence de collisions, la dépendance en $\delta$ et $\mathbf{v}$ de $^{(1)} \sigma_{\mathrm{gq} 1}^{\left(k_{1}\right)}$ est celle de $\Gamma_{1}^{\prime}$. On a donc :

$$
{ }_{0}^{(1)} \sigma_{\mathrm{g} q_{1}}^{\left(k_{1}\right)}(-\delta,-\mathbf{v})={ }_{0}^{(1)} \sigma_{\mathrm{g} q_{1}}^{\left(k_{1}\right)}(\delta, \mathbf{v}) \text {. }
$$

Pour le terme à 1 collision :

$$
\begin{aligned}
\underset{1}{(1)} \sigma_{\mathrm{g} q_{1}}^{\left(k_{1}\right)}(-\delta,-\mathbf{v}) & =\iint_{-\infty}^{+\infty} \mathrm{d}^{2}\left(-\mathbf{v}^{\prime}\right) \frac{K^{\left(k_{1}\right)}\left(-\mathbf{v}^{\prime},-\mathbf{v}\right){ }_{0}^{(1)} \sigma_{\mathrm{gq} 1}^{\left(k_{1}\right)}\left(-\delta,-\mathbf{v}^{\prime}\right)}{i q_{1} \omega_{0}+\gamma} \\
& =\iint_{-\infty}^{+\infty} \mathrm{d}^{2} \mathbf{v}^{\prime} \frac{K^{\left(k_{1}\right)}\left(-\mathbf{v}^{\prime},-\mathbf{v}\right){ }_{0}^{(1)} \sigma_{g q_{1}}^{\left(k_{1}\right)}\left(\delta, \mathbf{v}^{\prime}\right)}{i q_{1} \omega_{0}+\gamma}
\end{aligned}
$$

Le noyau de collision $K^{\left(k_{1}\right)}\left(-\mathbf{v}^{\prime},-\mathbf{v}\right)$ est égal à $K^{\left(k_{1}\right)}\left(\mathbf{v}^{\prime}, \mathbf{v}\right)$ (rotation de $\pi$ dans le plan $\left.z \mathrm{O} x\right)$. On voit donc que :

$$
{ }_{1}^{(1)} \sigma_{\mathrm{gq} 1}^{\left(k_{1}\right)}(-\delta,-\mathbf{v})={ }_{1}^{(1)} \sigma_{\mathrm{gq} 1}^{\left(k_{1}\right)}(\delta, \mathrm{v})
$$

et ce résultat se généralise immédiatement à $n$ quelconque, de sorte que :

$$
{ }^{(1)} \sigma_{q_{1}}^{\left(k_{1}\right)}(-\delta,-\mathbf{v})={ }^{(1)} \sigma_{q_{1}}^{\left(k_{1}\right)}(\delta, \mathbf{v}) \text {. }
$$

Au deuxième ordre en intensité, et pour un signal de " pompage-déplacement ", on a :

$$
{ }_{0}^{(2)} \sigma_{\mathrm{gQ}}^{(\mathbf{K})}(-\delta,-\mathbf{v})=-{ }_{0}^{(2)} \sigma_{\mathrm{gQ}}^{(K)}(\delta, \mathbf{v})
$$

puisque

$$
\Delta E^{\prime}(-\delta,-\mathbf{v})=-\Delta E^{\prime}(\delta, \mathbf{v})
$$

et ce résultat se généralise à tous les ordres en $p$, par un raisonnement identique à celui que nous venons de faire.

Le signal détecté vérifie donc :

$$
S(-\delta)=\iint_{-\infty}^{+\infty} \mathrm{d}^{2} v \mathfrak{D}(-\delta,-\mathbf{v}){ }_{n, p}^{(2)} \sigma_{g Q}^{(K)}(-\delta,-v)=-S(\delta)
$$

puisque la fonction $D$ est invariante quand on change $\delta$ en $-\delta$ et $v$ en $-\mathbf{v}$. 
En résumé, nous avons montré que les signaux induits par les collisions qui conservent partiellement les observables créées par le pompage, ont la même parité par rapport à $\delta=\Omega_{\mathrm{L}}-\Omega_{0}$, que ceux qu'on obtient en l'absence de collisions. Ils sont donc pairs pour des signaux de " pompage-pompage ", et impairs pour des signaux de " pompage-déplacement ». Bien entendu, leur profil est plus large que celui qu'on obtiendrait sans collisions, et il dépend de la forme du noyau; cette dépendance est discutée dans [11] pour le pompage optique sélectif en vitesses par un seul faisceau.

4. Dépendance en champ magnétique ; exemple simple. - Pour montrer comment la dépendance en champ magnétique des signaux induits par les collisions peut être calculée à partir des expressions écrites ci-dessus, nous allons choisir l'exemple de l'orientation longitudinale créée par un effet croisé de " pompage-déplacement » à partir de deux orientations (premier effet du tableau III).

En l'absence de collisions, cet effet, qui provient de deux termes d'amplitudes égales en $\left[T_{-1}^{(1)}, T_{1}^{(1)}\right]$ et $\left[T_{1}^{(1)}, T_{-1}^{(1)}\right]$, a pour dépendance en champ magnétique (cf. 2.3.4) :

$$
i\left(\frac{1}{\gamma+i \omega_{0}}-\frac{1}{\gamma-i \omega_{0}}\right)=\frac{2 \omega_{0}}{\gamma^{2}+\omega_{0}^{2}} .
$$

Les deux termes dans la parenthèse correspondent aux dépendances en champ magnétique des orientations transversales $T_{1}^{(1)}$ et $T_{-1}^{(1)}$ créées de façon intermédiaire par le premier cycle de pompage optique.

Intéressons-nous maintenant au terme à une collision dans cet effet $(n+p=1)$. Deux cas sont à envisager :

- Si la collision a lieu après le deuxième cycle de pompage $(p=1)$ : non seulement la dépendance en champ magnétique des termes intermédiaires ${ }^{(1)} \sigma_{\mathrm{g} \pm 1}^{(1)}$ est inchangée puisque $n=0$, mais de plus celle de ${ }^{(2)} \sigma_{\mathrm{g} 0}^{(1)}$ l'est également puisque l'observable obtenue après le deuxième cycle de pompage est longitudinale et n'évolue pas dans le champ magnétique $(Q=0)$. Finalement, la dépendance en champ est la même que sans collisions (ce résultat se généralise immédiatement à $p$ quelconque pourvu que $n=0$ ).

- Si la collision a lieu entre les deux cycles de pompage $(n=1)$, les termes intermédiaires ${ }_{1}^{(1)} \sigma_{\mathrm{g} \pm 1}^{(1)}$ varient maintenant comme $\frac{1}{\left(\gamma \pm i \omega_{0}\right)^{2}}$ et l'effet au deuxième ordre a donc pour dépendance en champ :

$$
i\left[\frac{1}{\left(\gamma+i \omega_{0}\right)^{2}}-\frac{1}{\left(\gamma-i \omega_{0}\right)^{2}}\right]=\frac{4 \gamma \omega_{0}}{\left(\gamma^{2}+\omega_{0}^{2}\right)^{2}} .
$$

Il s'agit toujours d'une fonction impaire en champ magnétique, mais qui est plus étroite que la précédente. Pour la même raison que plus haut, on aurait trouvé la même dépendance pour $n=1$ et $p$ quelconque.

Un calcul analogue peut être fait pour un nombre total de collisions plus grand que 1. Dans le cas considéré, seul intervient $n$, et la dépendance en champ magnétique est :

$$
i\left[\frac{1}{\left(\gamma+i \omega_{0}\right)^{n+1}}-\frac{1}{\left(\gamma-i \omega_{0}\right)^{n+1}}\right] .
$$

Le cas choisi est particulièrement simple car la grandeur créée après deux cycles de pompage est longitudinale : elle n'évolue pas dans le champ magnétique et seules les collisions ayant lieu entre les deux cycles de pompage interviennent dans la dépendance en champ magnétique. De plus, cette dépendance s'écrit simplement car l'effet n'est la somme que de deux termes correspondant à $q= \pm 1$.

\section{Bibliographie}

[1] Voir par exemple Letoknov, V. S. et Chebotaiev, V. P., Non-Linear Laser Spectroscopy (Springer Verlag, Berlin) 1977.

[2] Pinard, M., Aminoff, C. G. et Lalö̈, F., Phys. Rev. $A 19$ (1979) 2366.

PinARD, M., Thèse d'Etat, Paris (1980).

[3] Barrat, J. P. et Cohen-Tannoudj, C., J. Physique Radium 22 (1961) 329 et 443.

Cohen-Tannoudj, C., Thèse d'Etat, Ann. Phys. 7 (1962) 423 et 469.

[4] Happer, W., Rev. Mod. Phys. 44 (1972) 169.

[5] Cohen-Tannoudi, C., dans Les Houches 1975, Fron- tiers in Laser Spectroscopy, Vol. 1, édité par R. Balian, S. Haroche et S. Liberman (NorthHolland, Amsterdam) 1977, p. 3.

[6] Cohen-Tannoudi, C. et Lalö, F., J. Physique 28 (1967) 505 et 722.

[7] Julien, L., Thèse d'Etat, Paris (1981).

[8] Kolchenko, A. P., Pukhov, A. A., Rautian, S. G. et Shalagin, A. M., Sov. Phys. J.E.T.P. 36 (1973) 619.

[9] Berman, P. R., Phys. Rev. A 13 (1976) 2191.

[10] Brechignac, C., Vetter, R. et Berman, P. R., Phys. Rev. A 17 (1978) 1609. 
[11] Aminoff, C. G. et Pinard, M., J. Physique 43 (1982) 263.

[12] Gorlicki, M., Peuriot, A. et Dumont, M., J. Physique Lett. 41 (1980) L-275.

[13] Cohen-Tannoudi, C. et Dupont-Roc, J., Phys. Rev. A 5 (1972) 968.

Dupont-Roc, J., Thèse d'Etat, Paris (1972).

[14] Julien, L., Pinard, M. et Lalö̈, F., Phys. Rev. Lett. 47 (1981) 564.

[15] Cohen-Tannoudi, C., dans Atomic Physics 4, édité par G. Zu Putlitz, E. W. Weber et A. Winnacker (Plenum Press, New York) 1975, p. 589.

[16] Ducloy, M., Thèse de 3e cycle, Paris (1968).

[17] FANO, U. et RACAH, G., "Irreductible Tensorial Sets " (Academic Press Inc., New York) 1959.

[18] Oмоnт, A., Prog. Quant. Electron. 5 (1977) 69.
[19] Lalö̈, F., Leduc, M. et Minguzzi, P., J. Physique 30 (1969) 277 et 341.

[20] Faroux, J. P., Thèse d'Etat, Paris (1969).

[21] Messiah, A., "Mécanique Quantique " tome II (Ed. Dunod, Paris) $1959^{\circ}$.

[22] Edmonds, A. R., "Angular Momentum in Quantum Mechanics » (Princeton) 1957.

[23] Lombardi, M., J. Physique 30 (1969) 631.

[24] Cohen-Tannoudi, C., et Dupont-Roc, J., Opt. Commun. 1 (1969) 184.

[25] Grandin, J. P., Hennecart, D., Husson, X., LeCler, D., VienNe, J. F. et Barrat-Rambosson, M., J. Physique 36 (1975) 787.

[26] Pinard, M., Aminoff, C. G. et Lalö̈, F., Appl. Phys. 15 (1978) 371.

Aminoff, C. G. et Kaivola, M., Opt. Commun. 37 (1981) 133. 